\title{
Moduli space holography and the finiteness of flux vacua
}

\author{
Thomas W. Grimm \\ Institute for Theoretical Physics Utrecht University, \\ Princetonplein 5, 3584 CE Utrecht, The Netherlands \\ E-mail: t.w.grimm@uu.nl
}

ABSTRACT: A holographic perspective to study and characterize field spaces that arise in string compactifications is suggested. A concrete correspondence is developed by studying two-dimensional moduli spaces in supersymmetric string compactifications. It is proposed that there exist theories on the boundaries of each moduli space, whose crucial data are given by a Hilbert space, an $S l(2, \mathbb{C})$-algebra, and two special operators. This boundary data is motivated by asymptotic Hodge theory and the fact that the physical metric on the moduli space of Calabi-Yau manifolds asymptotes near any infinite distance boundary to a Poincaré metric with $S l(2, \mathbb{R})$ isometry. The crucial part of the bulk theory on the moduli space is a sigma model for group-valued matter fields. It is discussed how this might be coupled to a two-dimensional gravity theory. The classical bulk-boundary matching is then given by the proof of the famous $S l(2)$ orbit theorem of Hodge theory, which is reformulated in a more physical language. Applying this correspondence to the flux landscape in Calabi-Yau fourfold compactifications it is shown that there are no infinite tails of self-dual flux vacua near any co-dimension one boundary. This finiteness result is a consequence of the constraints on the near boundary expansion of the bulk solutions that match to the boundary data. It is also pointed out that there is a striking connection of the finiteness result for supersymmetric flux vacua and the Hodge conjecture.

Keywords: Differential and Algebraic Geometry, Flux compactifications, Sigma Models, Superstring Vacua

ARXIV EPRINT: 2010.15838 


\section{Contents}

1 Introduction 1

2 Motivation using asymptotic Hodge theory $\quad 6$

2.1 Hodge norm and Hodge decomposition in the bulk 6

2.2 Near-bounday expansions and nilpotent orbits 8

$\begin{array}{lll}2.3 & \text { Differential equations and constraints from nilpotent orbits } & 11\end{array}$

$\begin{array}{ll}2.4 & \text { Metric on the moduli space and its near boundary expansion }\end{array}$

3 The bulk theory on the moduli space $\quad 17$

$\begin{array}{lll}3.1 \text { Bulk action for matter fields } & 17\end{array}$

$\begin{array}{ll}3.2 & \text { On the coupling to gravity } \\ \end{array}$

4 The $\operatorname{Sl}(2, \mathbb{C})$ boundary theory $\quad 22$

4.1 Boundary charge operator and $\mathfrak{s l}(2, \mathbb{C})$-algebra 23

$\begin{array}{lll}4.2 & \text { Boundary eigenstates } & 26\end{array}$

4.3 Boundary operators and the phase operator 28

$\begin{array}{ll}4.4 \text { Classification of boundary theories } & 29\end{array}$

5 Bulk-boundary correspondence $\quad 30$

5.1 Bulk theory solutions as series expansions $\quad 32$

$\begin{array}{lll}5.2 & \text { Asymptotic expansions of the matter fields } 36\end{array}$

$\begin{array}{lll}5.3 & \text { Uniqueness of the near boundary solution } & 38\end{array}$

6 The finiteness of the flux landscape and the distance conjecture 42

6.1 Leading behaviour of the Hodge norm 42

6.2 Proving the finiteness of the flux landscape 43

6.3 Comments on the distance conjecture 48

$\begin{array}{lll}7 & \text { Conclusions and further discussions } & 49\end{array}$

A Computing the phase operator for a nilpotent orbit $\quad 52$

\section{Introduction}

The search for general principles that identify effective theories that can be consistently coupled to quantum gravity has recently attracted much attention [1]. These principles have been formulated in a number of quantum gravity or 'swampland' conjectures. A motivation for this work provides the so-called distance conjecture [2]. It deals with effective theories with scalar fields and suggests that, if a UV completion with gravity exists, it has to admit 
states with a certain universal behaviour when approaching points in field space that are at infinite shortest geodesic distance. The profoundness of this conjecture arises from the fact that it links properties of the field space to the existence of certain states in the underlying theory near such infinite distance points. Even if one might not know the whole spectrum of states, at least a subsector has to follow a rather constrained asymptotic behaviour. In this work, we suggest that this asymptotic structure is dictated by a holographic principle and the existence of an auxiliary boundary theory living at infinite distance boundaries of field space.

The physical motivation for the holographic dictionary lies largely in the distance conjecture combined with the observation that the asymptotic field space metric and the relevant towers of states follow stringent constraints in all known examples arising from string compactifications [3-16]. Furthermore, it is intriguing to interpret the distance conjecture as describing a mechanism of ensuring that exact global symmetries are absent in any gravity-coupled theory with finitely many states $[17,18]$. Adapting the converse perspective, we claim that at any infinite distance boundary in field space a global symmetry arises and that we can attach this data to the boundary. Our intuition is then derived from the expectation that combining the existence of a global symmetry with all possible positivity constraints, e.g. of the field space metric, will strongly restrict the asymptotic behaviour of the theory. That this is indeed the case in supersymmetric string compactifications was recently highlighted in $[3,6,7,11,15,19]$. In fact, in the vector sector of $\mathcal{N}=2$ string compactifications it is the asymptotic global symmetry and the positivity of the physical couplings and masses in the asymptotic regime that fixes much of the asymptotic structure. The underlying mathematical reason for this observation can be described using asymptotic Hodge theory [20, 21]. This broad and abstract theory will allow us to develop the holographic dictionary to a significant extend.

The detailed construction that we will present is motivated by an in-depth understanding of the field spaces that arise in string compactifications on Calabi-Yau manifolds. More precisely, we start our discussion with a study of the asymptotic behaviour of the moduli space of geometric deformations that preserve the Calabi-Yau condition and later strip away the underlying geometric motivation. The geometric settings have been studied intensively in the past and it is well-known that there are two types of deformations of Calabi-Yau geometries, the complex structure deformations and the Kähler structure deformations. Since by mirror symmetry the latter deformations can be realized as a special subset of the former [22], we will exclusively focus on complex structure deformation space in this work. It is central to this work that the complex structure moduli space has boundaries. These correspond to choices of complex structures for which the Calabi-Yau manifold degenerates. While some of these degeneration points, such as the large complex structure point, have been studied in much detail in the past, it is important to stress that there are a plethora of such degenerations and there is no detailed classification of the possibilities yet (see $[23,24]$ for recent progress). The crucial point is that at the boundaries of moduli space the associated manifold is so singular that the usual geometric structures, such as the Hodge norms determining the kinetic terms of fields, degenerate and can no longer be applied. Asymptotic Hodge theory shows, however, that there is a more abstract structure 
living on the boundary and we claim that this structure can be thought of as defining parts of a boundary theory. We will descibe in this work which set of boundary data determines the behaviour of the couplings in the effective theory and the moduli space close to boundary. In this geometric picture we thus find that when tuning the Calabi-Yau space to become singular such that a global symmetry emerges in moduli space, the structure of the effective theory and the moduli space is largely fixed by global symmetry, positivity, and holomorphicity. While our findings are compatible with the expectations from the distance conjecture at boundaries that are at infinite distance, we will see that the constrained asymptotic behaviour arises more generally.

The existence of a holographic description of the Calabi-Yau moduli space can also be motivated by noting that the physical metric on moduli space, i.e. the Weil-Petersson metric which arises in string compactifications, always asymptotes to a metric containing the factors of Poincaré metric at any infinite distance boundary [25]. In a real two-dimensional setting, which will be the main focus of this work, this means that the physical metric asymptotes to the two-dimensional Poincaré metric which is a patch of Euclidean $\mathrm{AdS}_{2}$. This metric has an $\mathfrak{s l}(2, \mathbb{R})$ isometry algebra that will non-trivially translate to a global symmetry algebra $\mathfrak{s l}(2, \mathbb{C})$ on the boundary. We will see that this boundary $\mathfrak{s l}(2, \mathbb{C})$ is indeed a result of the emerging global symmetry and exists more generally even if the boundary is not at infinite distance. The key quantity relevant to evaluate the asymptotic physical metric is the period matrix of the Calabi-Yau manifold. It encodes how the Hodge decomposition over the middle cohomology changes when moving over the complex structure moduli space. A remarkable result of Schmid [26] states that asymptotically this information is captured by a so-called nilpotent orbit, which packages the asymptotic behaviour in a seemingly simple polynomial way. For example, in the large complex structure or large volume boundary the nilpotent orbit captures this information in the periods remaining after dropping all exponential corrections. ${ }^{1}$ The nilpotent orbits will serve as the motivation for the bulk and boundary theories that we discuss in this work. Eventually, however, the results can be formulated without any reference to nilpotent orbits. They arise as solutions to the bulk theory that match the boundary data. We will call such solutions 'physical' in the following, since they appear in actual geometric compacitifactions. Viewed abstractly, however, both the bulk and boundary data can then be formulated without reference to a geometric string theory setting.

In order to construct the bulk action we restrict our attention to real two-dimensional field spaces. In other words, the field space of the effective theory will thus be viewed as a two-dimensional worldsheet. Ideally we would like to construct a gravity model coupled to a sigma-model on the worldsheet. The matter sector of this theory will be constrained by field equations that also arise in asymptotic Hodge theory. It is known from [26, 27] that nilpotent orbits in one complex dimension provide solutions to Nahm's equations that satisfy a certain constraint and match a well-defined set of boundary conditions. An action principle associated to Nahm's equations was discussed long ago in [28] and we will

\footnotetext{
${ }^{1}$ We stress that the nilpotent orbit exists near every boundary and, in general, indirectly captures exponential corrections that are needed for the positivity of the Hodge norm in the asymptotic regime.
} 
generalize it to a sigma model action on the worldsheet. A significant generalization of this action to the multiple variables $t t^{*}$-system of [29] appeared more recently in [30]. We also comment on the coupling of two-dimensional gravity to the matter sector. This is similar in spirit to the suggestion by Cecotti [31], who proposes to couple this sigma model to Einstein gravity for higher-dimensional worldsheets. ${ }^{2}$ While we will not present a complete action principle, we will successively build up a set of field equations. These turn out to admit solutions that are the nilpotent orbits that can arise in Calabi-Yau compactifications, together with the physical metric on moduli space. Crucially this requires to fix boundary conditions which we propose stem from a boundary theory.

To motivate the existence of a boundary theory we will again start with a nilpotent orbit, which we consider as the physical solutions to the bulk theory, and extract the data on the boundary that fixes such solutions. In order to do that we will use the famous $\mathrm{Sl}(2)$ orbit theorem of Schmid [26] and Cattani, Kaplan, and Schmid [27]. The set of boundary data will consist of an $\mathfrak{s l}(2, \mathbb{C})$ symmetry algebra acting on a finite-dimensional Hilbert space. The latter can be obtained as complexification of the charge or flux lattice relevant in the effective theory, and corresponds in geometric setting to the middle cohomology group of the Calabi-Yau manifold. The Hilbert space has a special $\mathfrak{s l}(2, \mathbb{C})$-compatible norm that is induced by an operator $Q_{\infty}$. Geometrically this $Q_{\infty}$ defines a Hodge decomposition that exists on the boundary of the moduli space despite the fact that the corresponding compactification geometry is badly singular $[26,27]$. The $\mathfrak{s l}(2, \mathbb{C})$ algebra turns out to be non-trivially related to the global symmetry in the asymptotic bulk solutions. We describe that boundary data contains a real nilpotent operator, which we call phase operator, that encodes how the asymptotic global symmetry is rotated into the boundary $\mathfrak{s l}(2, \mathbb{C})$ and how positivity constraints on the bulk solution map to the boundary. The additional information contained in the phase operator turns out to be central to the whole construction. ${ }^{3}$ Taken together this boundary data will suffice for our construction and serves as evidence for the existence of a boundary theory. Further evidence for the existence of such a boundary theory is provided by a number of conjectures put forward in [3, 12], and [33, 34], each discussing aspects of the theories that might emerge at infinite distance boundaries. While we will leave its full construction to future work, we will sometimes refer to the boundary data as describing a boundary theory.

Reconstructing the bulk solutions matching the boundary data turns out to be highly non-trivial and contained in the proof of the $S l(2)$ orbit theorem [26, 27]. Remarkably, the aforementioned boundary data specifies the bulk solution uniquely. To see this we will solve the matter equations of motion with a near boundary expansion and then determine their properties and eventually their dependence on the boundary operators. The constraints on the coefficients arise from the $\mathfrak{s l}(2, \mathbb{C})$ symmetry and will turn out to be central in the finiteness proof that we discuss in the last part of this work. In showing that there is a unique reconstruction of the bulk solution from the boundary data, we discuss how the phase operator becomes of crucial importance. We determine a single matrix equation (5.4) which

\footnotetext{
${ }^{2}$ The described perspective has been developed independently.

${ }^{3}$ In Hodge theory this operator was introduced by Deligne [27, 32] as a unique rotation of any complex mixed Hodge structure into a mixed Hodge structure split over the real numbers.
} 
provides the unique match [27]. It then follows that coefficients in any bulk solution matching to the boundary are universal non-commutative polynomials in the $\mathfrak{s l}(2, \mathbb{C})$ generators and the phase operator.

In the final part of this work we will highlight some first non-trivial physical applications of the holographic perspective by discussing the finiteness of the flux landscape and the validity of the distance conjecture. In particular, we address in detail the longstanding question about the finiteness of flux vacua in Type IIB and F-theory flux compactifications [35, 36]. Formulated in F-theory or M-theory language, such compactifications are specified by a Calabi-Yau fourfold with a background flux $G_{4}$. The classical equations of motion then demand that this flux is self-dual in a general vacuum, while consistency demands that the flux-square is bounded by a tadpole constraint. While in the bulk of the moduli space it easy to argue that there are only finitely many fluxes and self-dual loci in moduli space, these could accumulate near its boundaries [37, 38]. We show that this does not happen when approaching any co-dimension one boundary [39, 40]. The result derives from the described bulk-boundary construction and is a consequence of the fact that the near boundary expansion of the bulk solution is constraint by the boundary data to forbid infinite tails. To prove finiteness for all boundaries will be the aim of [40]. It is interesting to point out that in the supersymmetric case in which the $G_{4}$ fluxes are restricted to be of $(2,2)$-type a famous result [41] provides a general proof of finiteness near any boundary. The significance of the latter publication arises due to the fact that this result can also be obtained by assuming the Hodge conjecture. Restricted to the co-dimension one boundaries, the main tool of [41] is precisely the $S l(2)$ orbit theorem underlying the correspondence discussed here. This gives further support to the significance of the described structures and the power of this formalism.

This article is structured as follows. In section 2 we begin by motivating our constructions by recalling some facts about asymptotic Hodge theory. In particular, we describe how the Hodge decomposition of the cohomology groups of forms behaves near the boundary of moduli space and how this behaviour is captured by nilpotent orbits. We then discuss the asymptotic form of the Weil-Petersson and the Hodge metric on moduli space and show when they asymptote to the Poincaré metric near the boundaries. In section 3 we turn to the discussion of the bulk theory on moduli space. We formulate field equations and an action principle for group-valued matter fields and discuss aspects of coupling this theory to gravity. Important aspects of the boundary theory are then discussed in section 4, where it is explained how a set of boundary data is fixed by symmetry and positivity. Technically most involved is section 5 , in which we describe how the boundary data singles out special sets of bulk solutions and constrains their behaviour. It contains some of the key steps of the proof of the $S l(2)$-orbit theorem reformulated to support the holographic perspective. In the final section 6 we then apply these finding to address the finiteness of flux vacua on Calabi-Yau fourfolds. We show the finiteness of self-dual fluxes near co-dimension one boundaries and comment on the finiteness of $(2,2)$-fluxes. We close with some remarks on applying the holographic perspective to the distance conjecture. The paper contains one appendix A discussing the computation of the phase operator. 


\section{Motivation using asymptotic Hodge theory}

In this section we provide the motivation for the construction of the bulk theory and the bulk-boundary matching by introducing some results from asymptotic Hodge theory. While many of the described facts are true for general Kähler manifolds, we will restrict our attention to complex $D$-dimensional Calabi-Yau manifolds $Y_{D}$. In this cases, the geometry of the complex structure moduli space $\mathcal{M}$ of $Y_{D}$ can be encoded by the moduli dependence of the $(D, 0)$-form $\Omega$. We first introduce the Hodge norm and the Hodge decomposition in section 2.1 and comment on its relevance in string compactifications. In section 2.2 we then restrict our attention to the near boundary region in $\mathcal{M}$. We explain how the Hodge decomposition near the boundary can always be encoded by a expansion that is polynomial in the moduli and is best described by a so-called nilpotent orbit. In passing we argue that this expansion nevertheless encodes 'non-perturbative' terms in the periods of $\Omega$ at most boundaries. Crucial for developing the bulk theory is the fact that the nilpotent orbits satisfy a set of differential equations. We introduce these equations in section 2.3, point out their relation to Nahm's equations, and discuss an associated action principle. Finally, in section 2.4, we introduce two metrics on the moduli space $\mathcal{M}$ and discuss their near boundary expansion. The first one is the Weil-Petersson metric and is the physical metric in string compactifications on $\mathcal{M}$. The second one is the Hodge metric and closely related to the Hodge norm. We note that the asymptotic form of the Weil-Peterson metrics contains a Poincaré metric at all infinite distance boundaries, while this fact is more generally true for the Hodge metric. The isometry group $S l(2, \mathbb{R})$ of the Poincaré metric will translate to part of the symmetry group found in the boundary theory in section 4 .

\subsection{Hodge norm and Hodge decomposition in the bulk}

In order to introduce a holographic picture of the moduli space we first have to specify which quantities we want to keep track of. Let us denote by $Y_{D}$ a compact Calabi-Yau manifold of complex dimension $D$. For concreteness we will set our focus on the behaviour of the Hodge norm of a $D$-form cohomology class of $Y_{D}$. Considering two elements $\alpha, \beta \in H^{D}\left(Y_{D}, \mathbb{C}\right)$, the Hodge norm arises from the inner product

$$
\int_{Y_{D}} \bar{\alpha} \wedge * \beta=\frac{1}{D !} \int_{Y_{D}} d^{2 D} x \sqrt{\operatorname{det} g} \bar{\alpha}_{\mu_{1} \ldots \mu_{D}} \beta^{\mu_{1} \ldots \mu_{D}}
$$

and will be denoted by

$$
\|\alpha\|^{2}=\int_{Y_{D}} \bar{\alpha} \wedge * \alpha .
$$

Note that the inner product (2.1) is induced by the Hodge norm and therefore it often suffices to discuss the latter. In addition to the inner product induced by the norm we can also define the wedge-product ${ }^{4}$

$$
\langle\alpha, \beta\rangle:=\int_{Y_{D}} \alpha \wedge \beta
$$

\footnotetext{
${ }^{4}$ Note that in the mathematical literature $[26,27]$ this inner product is denoted by $S(\alpha, \beta)=\langle\beta, \alpha\rangle$.
} 
which is symmetric for $D$ even and skew-symmetric for $D$ odd. It will be important in the following to consider transformations $g$ preserving $\langle\cdot, \cdot\rangle$. The group of such transformations over the real numbers will be denoted by $G_{\mathbb{R}}$, while the corresponding algebra is denoted by $\mathfrak{g}_{\mathbb{R}}$. Hence, we have

$$
g \in G_{\mathbb{R}}: \quad\langle g \alpha, \beta\rangle=\left\langle\alpha, g^{-1} \beta\right\rangle, \quad L \in \mathfrak{g}_{\mathbb{R}}: \quad\langle L \alpha, \beta\rangle=-\langle\alpha, L \beta\rangle .
$$

As an example, we note that for Calabi-Yau threefolds one has $G_{\mathbb{R}}=S p\left(2 h^{2,1}+2, \mathbb{R}\right)$. The complex version of this group and algebra are henceforth denoted by $G_{\mathbb{C}}, \mathfrak{g}_{\mathbb{C}}$.

When computing the effective actions arising from compactifications of string theory the Hodge norm (2.2) appears in many instances. As a first example, note that in Type IIB string theory on a Calabi-Yau threefold the Hodge norm determines the kinetic terms of the four-dimensional gauge fields, which arise by expanding the R-R four-form $C_{4}$ into threeforms $H^{3}\left(Y_{3}, \mathbb{Z}\right)$. Picking a symplectic basis $\left(\alpha_{M}, \beta^{N}\right)$ with $\left\langle\alpha_{M}, \beta^{N}\right\rangle=\delta_{M}^{N}$ of $H^{3}\left(Y_{3}, \mathbb{Z}\right)$ we write $C_{4}=A^{M} \wedge \alpha_{M}-\tilde{A}_{M} \wedge \beta^{M}$. The four-dimensional vectors $A^{M}$ and $\tilde{A}_{M}$ are electric and magnetic $U(1)$ gauge fields in the effective theory, respectively. The charged particles in the effective theory arise from D3-branes wrapped on three-cycles in $Y_{3}$. The space $H^{3}\left(Y_{3}, \mathbb{Z}\right)$ can be identified with the charge lattice of these states under $\left(A^{M}, \tilde{A}_{N}\right)$. The relevance of these states in the distance conjecture will be briefly discussed in the very last section 6.3. A second example, which will be central to section 6, are F-theory and M-theory compactifications on Calabi-Yau fourfolds. In these cases the flux scalar potential induced by a background four-form flux $G_{4}$ in $H^{4}\left(Y_{4}, \mathbb{Z} / 2\right)$ is determined by the Hodge norm. The lattice $H^{4}\left(Y_{4}, \mathbb{Z} / 2\right)$ corresponds to the flux lattice.

The goal of the following discussion is to keep track of the dependence of the Hodge norm on the complex structure deformations of the manifold $Y_{D}$. For Calabi-Yau manifolds it can be shown that there exists an unobstructed moduli space $\mathcal{M}$, the complex structure deformation space. This space is a Kähler manifold of complex dimension $h^{D-1,1}=$ $\operatorname{dim} H^{D-1,1}\left(Y_{D}\right)$. In order to investigate the change of (2.2) along $\mathcal{M}$, we consider the Hodge decomposition

$$
H^{D}\left(Y_{D}, \mathbb{C}\right)=H^{D, 0} \oplus H^{D-1,1} \oplus \ldots \oplus H^{1, D-1} \oplus H^{0, D}
$$

where $\overline{H^{p, q}}=H^{q, p}$ and $p+q=D$. This decomposition has to be determined for the chosen complex structure on $Y_{D}$ and hence varies when moving along $\mathcal{M}$. Using the Kähler metric on $Y_{D}$ to determine the Hodge star $*$ one shows that

$$
* w^{p, q}=i^{p-q} w^{p, q}, \quad w^{p, q} \in H^{p, q} .
$$

Furthermore, one has the relation that

$$
\left\langle w^{p, q}, v^{r, s}\right\rangle=0, \quad \text { for } p \neq s, q \neq r .
$$

This implies that one can evaluate the Hodge norm $\|\alpha\|$, defined in $(2.2)$, if the $(p, q)$ decomposition of $\alpha$ has been determined. 


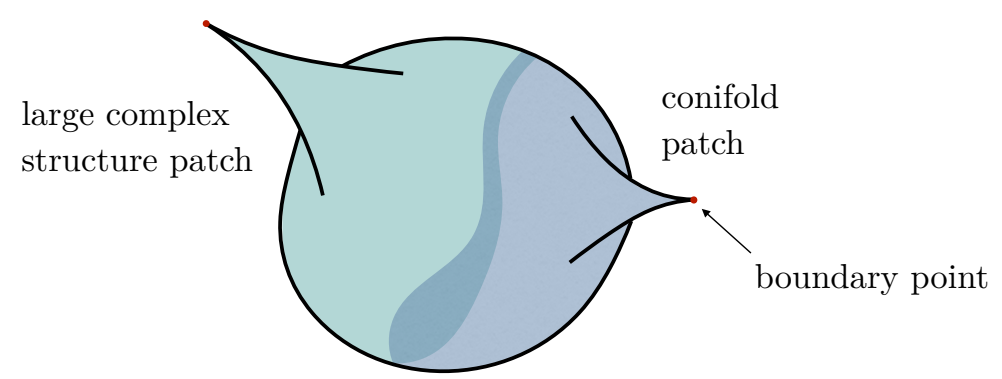

Figure 1: Schematic depiction of a complex one-dimensional moduli space. Two overlapping patches are indicated in light green and light blue. These can, for example, contain the large complex structure point and the conifold point of a $Y_{3}$.

The dependence of (2.2) on the coordinates $z^{I}$ of the moduli space $\mathcal{M}$ can thus be understood by following the $(p, q)$-decomposition along $\mathcal{M}$. It is actually better to study how the spaces

$$
F^{p}=\bigoplus_{r \geq p} H^{r, D-r}
$$

change when moving along $\mathcal{M}$. These spaces vary, at least locally, holomorphically in the complex coordinates $z^{I}$ of $\mathcal{M}$ [21]. The original decomposition (2.5) is then recovered by $H^{p, q}=F^{p} \cap \bar{F}^{q}$. The moduli dependence of the $F^{p}$ is, in general, given by complicated transcendental functions that solve partial differential equations known as the Picard-Fuchs equations. Generically the solutions have only a finite radius of convergence and, in order to cover the whole moduli space $\mathcal{M}$ one has to work in patches, leading to a picture as in figure 1.

It is crucial for our considerations to note that the moduli space $\mathcal{M}$ is, at first, neither smooth nor compact. This is due to the fact that, when changing the complex structure, the Calabi-Yau manifold can become so singular that a Hodge decomposition as in (2.5) no longer exists. Such singular loci constitute the boundaries of the moduli space. It was shown in $[42,43]$ that one can modify the boundary loci, by blowing up possible singularities, such that the boundary of $\mathcal{M}$ can be written as

$$
\partial \mathcal{M}=\bigcup_{k} \Delta_{k}
$$

where $\Delta_{k}$ are complex manifolds of complex dimension $h^{D-1,1}-1$ intersecting at normal instance. In the following sections, we will describe how an extended structure generalizing the decomposition (2.5) can be defined on the boundaries $\cup_{k} \Delta_{k}$. Before doing this, we study in more detail the near-boundary behaviour of the decomposition (2.5).

\subsection{Near-bounday expansions and nilpotent orbits}

In this subsection we discuss behaviour of the decomposition (2.5) near any boundary component $\partial \mathcal{M}$ of the moduli space $\mathcal{M}$. Recalling that the boundary splits into multiple $\Delta_{k}$, as discussed around (2.9), we want to consider a local patch containing a co-dimension 
(a.1)

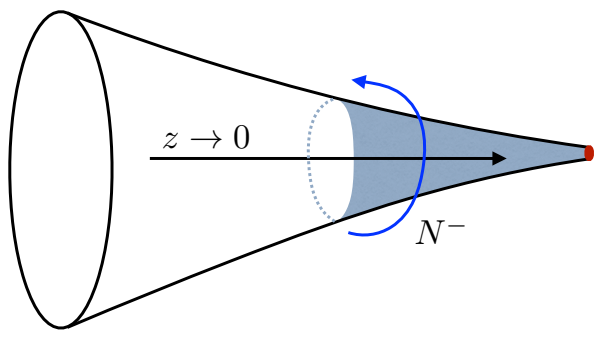

(a.2)

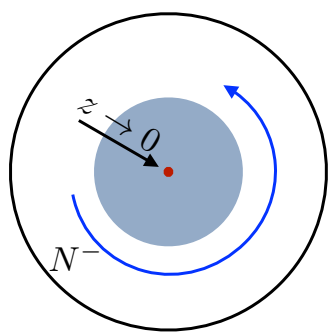

(b)

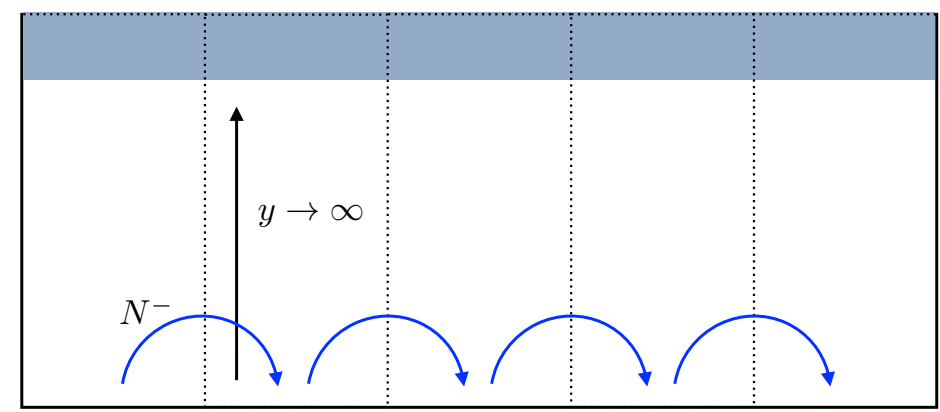

Figure 2: Schematic depiction of the asymptotic region in a complex one-dimensional moduli space. Figures (a.1) and (a.2) show the punctured disc parametrized by the complex coordinate $z$. The boundary of interest is the puncture at $z=0$. Figure (b) shows the universal cover of the punctured disc, the upper half plane parametrized by $t=x+i y$. The boundary of interest is now located at $y=\infty$. We also indicate that there can be a log-monodromy matrix $N^{-}$appearing in (2.12) when encircling the puncture or shifting in the upper-half plane.

$n$ boundary. We thus introduce local coordinates $z^{j} \equiv e^{2 \pi i t^{j}}, j=1, \ldots, n$, and $\zeta^{\kappa}$, such that the boundary component is approached in the limit

$$
z^{j} \rightarrow 0 \quad \text { or } \quad t^{j}=x^{j}+i y^{j} \rightarrow x_{0}^{j}+i \infty .
$$

Suppressing the $\zeta^{\kappa}$ coordinate directions, the considered configuration can be depicted as in figure 2.

We next recall the first major result of asymptotic Hodge theory, which states how the Hodge decomposition behaves for sufficiently large $y^{j} \gg 1$. As stated after (2.8) it is convenient to study the vector spaces $F^{p}$, since they vary holomorphically over the moduli space. This means that the $F^{p}$ are locally only depending on the coordinates $z^{j}$ or $t^{j}$ introduced in (2.10), but are independent of $\bar{z}^{j}, \bar{t}^{j}$. This statement extends to the complex coordinates $\zeta^{\kappa}$, which we will suppress in the following expressions. A main insight of Schmid [26] was that near a boundary $\operatorname{Im} t^{j}=y^{j} \rightarrow \infty$ the $F^{p}$ always take the form

$$
F^{p}(t) \approx F_{\mathrm{pol}}^{p}(t)
$$


where $F_{\mathrm{pol}}^{p}$ is varying, up to possibly an overall rescaling in any direction, as a polynomial in $t$. This 'polynomial part' is given by

$$
F_{\mathrm{pol}}^{p}(t)=e^{t^{j} N_{j}} F_{0}^{p}
$$

where $N_{j}$ are constant nilpotent matrices and the $F_{0}^{p}$ is independent of $t^{j}$, but can still depend holomorphically on $\zeta^{\kappa}$. The compatibility between the $N_{i}$ and the spaces $F_{0}^{p}$ can be stated as the condition that $N_{i} F_{0}^{p} \subset F_{0}^{p-1}$. The polynomial piece is known as the nilpotent orbit. Note that (2.11) is a relation between vector spaces and it is a non-trivial statement that the polynomial part alone has $\operatorname{dim} F^{p}=\operatorname{dim} F_{\text {pol }}^{p}$. Fixing a direction in the vector spaces there are generically exponentially suppressed corrections $\mathcal{O}\left(e^{2 \pi i t^{j}}\right)$ to the identification (2.11). These are strongly suppressed in the near-boundary regime $\operatorname{Im} t^{j} \gg 1$.

One can now show that (2.12) also defines a $(p, q)$-decomposition and an associated norm using the analog of (2.6). Concretely, we introduce the decomposition by

$$
H^{D}\left(Y_{D}, \mathbb{C}\right)=H_{\text {pol }}^{D, 0} \oplus H_{\mathrm{pol}}^{D-1,1} \oplus \ldots \oplus H_{\mathrm{pol}}^{0, D}
$$

by setting $H_{\mathrm{pol}}^{p, q}=F_{\mathrm{pol}}^{p} \cap \bar{F}_{\mathrm{pol}}^{q}$. A norm $\|\cdot\|_{\text {pol }}$ is then defined as

$$
\|\omega\|_{\mathrm{pol}}^{2}:=\left\langle\bar{\omega}, C_{\mathrm{pol}} \omega\right\rangle, \quad C_{\mathrm{pol}} \omega^{p, q}=i^{p-q} \omega^{p, q},
$$

where now $\omega^{p, q} \in H_{\mathrm{pol}}^{p, q}$. We also introduce the associated inner product

$$
\langle\omega \mid \nu\rangle_{\mathrm{pol}}:=\left\langle\bar{\omega}, C_{\mathrm{pol}} \nu\right\rangle,
$$

where we will use bra-ket notation when convenient. Note that it is non-trivial that the $H_{\text {pol }}^{p, q}$ obtained from the polynomial $F_{\text {pol }}^{p}$ suffice to span the whole space $H^{D}\left(Y_{D}, \mathbb{C}\right)$. In contrast, it is not true in general that $F_{0}^{p}$ can be used similarly to define a $(p, q)$-decomposition with a well-defined norm.

At first it seems natural to view $F_{\text {pol }}^{p}$ as only capturing 'perturbative terms', while the dropped corrections $\mathcal{O}\left(e^{2 \pi i t^{j}}\right)$ in (2.11) correspond to the non-perturbative corrections. While this interpretation can indeed be made more precise in the special limit known as the large complex structure limit, ${ }^{5}$ we will discuss in the following that this is not generally the case at other limits in moduli space. In fact, in most other situations, certain crucial non-perturbative corrections are captured by $F_{\mathrm{pol}}^{p}(t)$. To simplify the discussion let us assume that $\mathcal{M}$ is one-dimensional, i.e. we only use the coodinate $t$ to parametrize the Hodge decomposition. In order to see which information is captured by $F_{\text {pol }}^{p}$, we give an explicit expansion of the $(D, 0)$-form $\Omega$ spanning $F^{D}=H^{D, 0}$, which is a one-dimensional complex vector space for a Calabi-Yau manifold. Applying (2.11) with (2.12) to $F^{D}$, we find

$$
\Omega=e^{t N^{-}} a_{0}+e^{2 \pi i t} \tilde{a}_{1}+e^{4 \pi i t} \tilde{a}_{2}+\ldots
$$

\footnotetext{
${ }^{5}$ Mirror symmetry states that complex structure deformations are exchanged with Kähler structure deformations of a dual Calabi-Yau geometry [22]. In this dual picture the strings wrapping cycles in the dual space do induce perturbative and non-perturbative corrections with the above split.
} 
where we have fixed the overall normalization of $\Omega$ such that $a_{0}$ is independent of $t$. The derivative $\partial_{t} \Omega$ spans, together with $\Omega$ itself the space $F^{D-1}$ and takes the form

$$
\partial_{t} \Omega=e^{t N^{-}} N^{-} a_{0}+e^{2 \pi i t}\left(2 \pi i \tilde{a}_{1}+\partial_{t} \tilde{a}_{1}\right)+\ldots .
$$

Applying now (2.11) with (2.12) to $F^{D-1}$, we see that, as long as $N^{-} a_{0} \neq 0$, the first term in (2.17) takes again the form of a nilpotent orbit. However, if one considers a boundary with $N^{-} a_{0}=0$ then the exponential correction in (2.16) is actually needed, such that the whole space $H^{D}\left(Y_{D}, \mathbb{C}\right)$ can be obtained from the nilpotent orbit $F_{\mathrm{pol}}^{p}$. In this case $\partial_{t} \Omega$ is proportional to $e^{2 \pi i t}$, but this overall factor can be removed by a rescaling since (2.12) is an equality between vector spaces. In other words, the existence of a nilpotent orbit (2.12) that gives a splitting (2.13), implies that $\Omega$ should be expanded as

$$
\Omega=e^{t N^{-}}\left(a_{0}+e^{2 \pi i t} a_{1}+e^{4 \pi i t} a_{2}+\ldots\right)
$$

with the $a_{i}, i>0$ relevant as soon as the vectors $\left(N^{-}\right)^{n} a_{0}$ do not suffice to span $H^{D}\left(Y_{D}, \mathbb{C}\right)$. This implies that at many boundaries exponential corrections in $\Omega$ are implied by the existence of a nilpotent orbit with the above properties. This matches nicely the recent proposal put forward in [44] and will be discussed in more generality in [45]. We also note that the generic presence of exponential corrections has recently been shown in [30] by using other results from Hodge theory.

\subsection{Differential equations and constraints from nilpotent orbits}

With the motivation to develop a holographic perspective, it is desirable to formulate the behaviour of (2.5), and hence the norm (2.2), by introducing a set of fields that live on the moduli space $\mathcal{M}$ and admit equations of motions that are inspired by Hodge theory. In the following we will motivate such equations of motion by the conditions obeyed by the nilpotent orbit $F_{\text {pol }}^{p}$ introduced in section 2.2. This implies that we restrict our attention to the near boundary region. Eventually, the resulting equations can be considered without reference to an underlying nilpotent orbit.

To motivate the equations of motion let us again consider a one-dimensional limit (2.10), i.e. study the nilpotent orbit

$$
F_{\mathrm{pol}}^{p}(t)=e^{t N^{-}} F_{0}^{p},
$$

depending on one variable $t$. We want to study the moduli dependence of (2.19) with respect to a fixed Hodge decomposition $H_{\text {ref }}^{p, q}$. This decomposition could be picked by simply evaluating $F_{\text {poly }}^{p}(t)$ at a fixed $t_{0}$. However, we will make a more educated choice that prepares us for the discussion of the bulk-boundary correspondence that will follow below. In fact, we will consider a reference Hodge decomposition $H_{\mathrm{ref}}^{p, q}$ induced by

$$
F_{\text {ref }}^{p}=e^{i N^{-}} e^{-i \delta} F_{0}^{p}
$$

This choice corresponds to evaluating (2.19) at $t=i$ and multiply the result by a phase matrix $e^{-i \delta}$, here $\delta$ is a real matrix in $\mathfrak{g}_{\mathbb{R}}$ satisfying $\left[\delta, N^{-}\right]=0$. The matrix $\delta$ can be uniquely associated to a given $N^{-}, F_{0}^{p}$ and we will call $\delta$ the phase operator in the following. 
We will describe in appendix A how $\delta$ can be constructed starting from $N^{-}, F_{0}^{p}$. The reason why we extract a phase in (2.20) will become clear below. In a nutshell, we will find that asymptotically the moduli space metric admits an isometry group $\mathfrak{s l}(2, \mathbb{R})$, discussed after (2.44). The phase operator is extracted in such a way that one can find a real slice $H^{D}\left(Y_{D}, \mathbb{R}\right)$ in $H^{D}\left(Y_{D}, \mathbb{C}\right)$ on which $\mathfrak{s l}(2, \mathbb{R})$ acts faithfully. We also introduce the inner

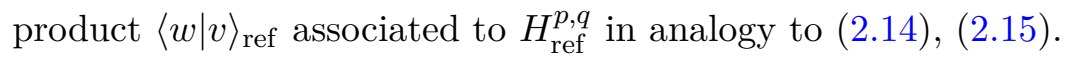

Starting from the reference splitting $H_{\text {ref }}^{p, q}=F_{\text {ref }}^{p} \cap \bar{F}_{\text {ref }}^{q}$ induced by (2.20), we will first restrict our attention to the imaginary part $\operatorname{Im} t=y$ that is taken to be large in (2.10) and

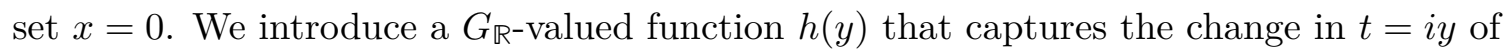
$H_{\mathrm{pol}}^{p, q}$ with respect to $H_{0}^{p, q}$ by setting

$$
H_{\mathrm{pol}}^{p, q}(i y)=h(y) H_{\mathrm{ref}}^{p, q}, \quad h(y) \in G_{\mathbb{R}},
$$

or

$$
F_{\mathrm{pol}}^{p}(i y)=e^{i y N^{-}} F_{0}^{p}=h(y) F_{\mathrm{ref}}^{p} .
$$

Recall that $G_{\mathbb{R}}$ is the real group keeping the inner product (2.3) invariant. Since $e^{x N^{-}}$is also an element of $G_{\mathbb{R}}$ one can complete (2.21) to

$$
H_{\mathrm{pol}}^{p, q}(t, \bar{t})=e^{x N^{-}} h(y) H_{\mathrm{ref}}^{p, q} \equiv \hat{h}(x, y) H_{\mathrm{ref}}^{p, q},
$$

where we have defined $\hat{h}(x, y):=e^{x N^{-}} h(y) \in G_{\mathbb{R}}$. We therefore get an explicit expression for the $(p, q)$-forms in the asymptotic regime, in terms of a reference splitting. While the dependence on $x$ is rather simple, the dependence on $y$ via $h(y)$ is generally involved. We therefore focus mostly on the $y$-dependence in the following, keeping in mind that the $x$-dependence can be restored rather easily.

We can also choose to encode the splitting $\mathcal{H}=\bigoplus_{p} H_{\text {ref }}^{p, D-p}$ by using an operator $Q$, which is independent of $t$. Such an operator is known as grading element $Q \in \mathfrak{g}_{\mathbb{C}}$, see e.g. $[46,47]$, and we will call it charge operator in the following. It is defined by ${ }^{6}$

$$
Q|w\rangle_{\mathrm{ref}}=\frac{1}{2}(2 p-D)|w\rangle_{\mathrm{ref}} \quad \text { for } \quad|w\rangle_{\mathrm{ref}} \in H_{\mathrm{ref}}^{p, D-p}
$$

where we are using a bra-ket notation for states in $\mathcal{H}$. Since $\overline{H_{\text {ref }}^{p, q}}=H_{\text {ref }}^{q, p}$ we conclude that

$$
\bar{Q}=-Q, \quad Q \in i \mathfrak{g}_{\mathbb{R}} .
$$

Recalling that the inner product $\langle w \mid v\rangle_{\text {ref }}$ is induced by a real Weil operator $C_{\text {ref }}$ as in (2.13), (2.14), we can use $Q$ to write

$$
\langle w \mid v\rangle_{\mathrm{ref}}=\left\langle w, C_{\mathrm{ref}} v\right\rangle, \quad C_{\mathrm{ref}}=i^{2 Q} .
$$

\footnotetext{
${ }^{6}$ As a side remark, we note that the action of $Q$ on operators as in (2.30) can also be formulated more mathematically by using the decomposition of the algebra $\mathfrak{g}_{\mathbb{C}}$, defined after $(2.3)$, induced by $H_{\text {ref }}^{p, q}$. Explicitly one splits $\mathfrak{g}_{\mathbb{C}}=\bigoplus_{p \in \mathbb{Z}} \mathfrak{g}_{\mathrm{ref}}^{p,-p}$ with $\mathfrak{g}_{\mathrm{ref}}^{p,-p}=\left\{T \in \mathfrak{g}_{\mathbb{C}}: T H_{\text {ref }}^{a, b}=H_{\text {ref }}^{a+p, b-p}\right\}$, where $\overline{\mathfrak{g}_{\text {ref }}^{p,-p}}=\mathfrak{g}_{\mathrm{ref}}^{-p, p}$ and $\left[\mathfrak{g}_{\text {ref }}^{p,-p}, \mathfrak{g}_{\text {ref }}^{q,-q}\right] \subset \mathfrak{g}_{\text {ref }}^{p+q,-(p+q)}$. The notation $\mathfrak{g}_{\text {ref }}^{p,-p}$ indicates that one is dealing with a Hodge structure of weight $p-p=0$. The action the charge operator $Q$ is then induced by the split as $[Q, T]=p T$ for $T \in \mathfrak{g}_{\mathrm{ref}}^{p,-p}$.
} 
The charge operator $Q$ will play a central role in the construction, since it encodes the Hodge decomposition. The fact that it is not a real operator implies that its eigenstates with real eigenvalues are complex.

The function $h(y)$ satisfies a set of equations that will constitute the base of the bulk theory discussed in section 3 . We will formulate these equations in the algebra $\mathfrak{g}_{\mathbb{R}}$ associated to $G_{\mathbb{R}}$ and thus define $\mathcal{N}^{0}(y), \mathcal{N}^{-}(y) \in \mathfrak{g}_{\mathbb{R}}$ by setting

$$
\mathcal{N}^{0}(y):=-2 h^{-1} \partial_{y} h, \quad \mathcal{N}^{-}(y):=h^{-1} N^{-} h, \quad \mathcal{N}^{+}(y):=\left(\mathcal{N}^{-}(y)\right)^{\dagger},
$$

where we abbreviate $\partial_{y}=\frac{d}{d y}$ and denote by $\dagger$ the operation to adjoin an operator with

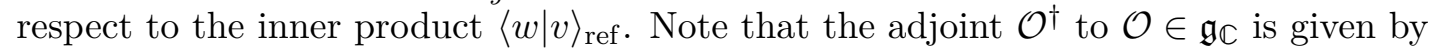

$$
\mathcal{O}^{\dagger}=-C_{\text {ref }}^{-1} \overline{\mathcal{O}} C_{\text {ref }}
$$

as can be seen from (2.15) and (2.4). Given these definitions one shows $[26]^{7}$ that any $\mathcal{N}^{0}(y), \mathcal{N}^{ \pm}(y)$ coming from a nilpotent orbit (2.19) obeys the differential equations

$$
\partial_{y} \mathcal{N}^{ \pm}= \pm \frac{1}{2}\left[\mathcal{N}^{ \pm}, \mathcal{N}^{0}\right], \quad \partial_{y} \mathcal{N}^{0}=-\left[\mathcal{N}^{+}, \mathcal{N}^{-}\right]
$$

as well as the algebraic relations ${ }^{8}$

$$
\left[Q, \mathcal{N}^{0}\right]=i\left(\mathcal{N}^{+}+\mathcal{N}^{-}\right), \quad\left[Q, \mathcal{N}^{ \pm}\right]=-\frac{i}{2} \mathcal{N}^{0}
$$

Note that from (2.26) and (2.30) we infer

$$
Q^{\dagger}=Q, \quad\left(\mathcal{N}^{0}\right)^{\dagger}=\mathcal{N}^{0}, \quad \mathcal{N}^{+}=\left(\mathcal{N}^{-}\right)^{\dagger},
$$

where we have chosen the last equality as definition of $\mathcal{N}^{+}$in (2.27). Note that the equations (2.29), (2.30) are both constraints on the function $h(y)$ and make no reference anymore to the nilpotent orbit.

It is not hard to check that the differential equations (2.29) are equivalent to Nahm's equations $\partial_{y} T_{i}=-\left[T_{j}, T_{k}\right]$, for every cyclic permutation of $i, j, k$. We can then use that in [28] it was argued that Nahm's equations (2.29) can be analyzed by using an action principle for the fields $h(y)$. The one-dimensional action reads

$$
\begin{aligned}
S_{\text {Nahm }}(h) & =\frac{1}{2} \int\left(\operatorname{Tr}\left|\left(h^{-1} \partial_{y} h\right)^{\dagger}+h^{-1} \partial_{y} h\right|^{2}+2 \operatorname{Tr}\left|h^{-1} N^{-} h\right|^{2}\right) d y \\
& =\frac{1}{2} \int\left(\frac{1}{4} \operatorname{Tr}\left|\left(\mathcal{N}^{0}\right)^{\dagger}+\mathcal{N}^{0}\right|^{2}+2 \operatorname{Tr}\left|\mathcal{N}^{-}\right|^{2}\right) d y
\end{aligned}
$$

where $|A|^{2}=A^{\dagger} A$, with the dagger and the trace is evaluated in the norm $\langle v \mid w\rangle_{\text {ref }}$. To see this, we check that the two equations to the left in (2.29) are automatically satisfied with

\footnotetext{
${ }^{7}$ This is shown in Lemma 9.8 .

${ }^{8}$ It might be useful to point out that [27] displays these conditions in a different form: $\mathcal{N}^{-}-\mathcal{N}^{+}$has charge $q=0$ under $\operatorname{ad}_{Q}$, while $\mathcal{N}^{0}$ has a $q=1$ and $q=-1$ component under ad $Q$. Furthermore, they require $\mathcal{N}^{-}+\mathcal{N}^{+}=i^{-Q} \mathcal{N}^{0} i^{Q}$, and $\mathcal{N}^{+}=-i^{-2 Q} \mathcal{N}^{-} i^{2 Q}$. These conditions are equivalent to the conditions stated here.
} 
the Ansatz (2.27), while the last one is obtained from (2.32). The latter statement follows if we vary the action by using $\delta \mathcal{N}^{-}=-\left[h^{-1} \delta h, \mathcal{N}^{-}\right]$and $\delta \mathcal{N}^{0}=-\left[h^{-1} \delta h, \mathcal{N}^{0}\right]-2 \frac{d}{d y}\left(h^{-1} \delta h\right)$. Note that we can equivalently formulate the conditions (2.29), (2.30) and the action (2.32) as constraints on $\hat{h}(x, y)$. We will see in section 3 that the construction naturally generalizes to an action for $\hat{h}(x, y)$ that can be coupled to two-dimensional gravity.

Before turning to the discussion of moduli space metric, let us close with a number of remarks. It is useful to note that the equations (2.29) can be rewritten in many different forms that highlight certain aspects of the construction. Firstly, we can use (2.30) to obtain a differential equation in Lax form,

$$
\partial_{y} \mathcal{N}^{-}=-i\left[\mathcal{N}^{-},\left[Q, \mathcal{N}^{-}\right]\right]
$$

which highlights the fact that there is an underlying integrable structure. Secondly, we can define the complex operators

$$
\mathcal{L}_{ \pm 1}(y):=\frac{1}{2}\left(\mathcal{N}^{+}+\mathcal{N}^{-} \mp i \mathcal{N}^{0}\right), \quad \mathcal{L}_{0}(y):=i\left(\mathcal{N}^{-}-\mathcal{N}^{+}\right)
$$

which satisfy $\mathcal{L}_{0}^{\dagger}=\mathcal{L}_{0}$ and $\mathcal{L}_{1}^{\dagger}=\mathcal{L}_{-1}$. The index $\alpha=(-1,0,1)$ indicates the charge of these operators,

$$
\left[Q, \mathcal{L}_{\alpha}\right]=\alpha \mathcal{L}_{\alpha}
$$

The equations (2.29) can then be written in the form of the $t t^{*}$ equations [29]. Setting $D \equiv \partial_{t}-\frac{i}{4} \mathcal{L}_{0}$ and $C \equiv \frac{1}{2} \mathcal{L}_{-1}$, the conditions (2.29) and (2.30) are then equivalent to

$$
\begin{array}{lll}
{[D, C]=[D, \bar{C}]=0,} & {[D, \bar{D}]=-[C, \bar{C}],} & \\
{[Q, C]=-C,} & {[Q, \bar{C}]=\bar{C},} & {[D, Q]=[\bar{D}, Q]=0 .}
\end{array}
$$

It is long known that these equations emerge from the variations of Hodge structures [29]. This connection also provides the link between our work and the recent papers [30, 31].

\subsection{Metric on the moduli space and its near boundary expansion}

There are at least two natural metrics, denoted by $g^{\mathrm{WP}}$ and $g^{\mathrm{H}}$, that can be defined on the moduli space $\mathcal{M}$. In Calabi-Yau compactifications the physically most relevant metric is the so-called Weil-Petersson metric $g^{\mathrm{WP}}$. It determines the kinetic terms of the complex coordinates $z^{K}$, when interpreting them as scalar fields in the low energy effective action. In the study of the distance conjecture in Calabi-Yau compactifications $g^{\mathrm{WP}}$ is therefore relevant to distinguish finite and infinite distance geodesics. As was shown in [25] and exploited in $[3,6]$, infinite distances only can occur when approaching a boundary with an associated non-vanishing $N^{-}$that satisfies $N^{-} a_{0} \neq 0$, with $a_{0}$ appearing in (2.16). We will see that the Weil-Petersson metric near such points takes a characteristic asymptotic form. In fact, we will argue that at each such limit an $\mathfrak{s l}(2, \mathbb{R})$ isometry algebra emerges. This feature turns out to be generally true for $N^{-} \neq 0$ if one considers the Hodge metric $g^{\mathrm{H}}$ on moduli space, which we will introduce below. Later on, in section 4, we will see that this $\mathfrak{s l}(2, \mathbb{R})$ is key in determining the symmetry of the boundary theory. 
To begin with, we recall that the Weil-Petersson metric is a Kähler metric and can be derived via $g_{I \bar{J}}^{\mathrm{WP}}=\partial_{z^{I}} \partial_{\bar{z}^{J}} K$ from a Kähler potential

$$
K=-\log i^{D} \int_{Y_{D}} \Omega \wedge \bar{\Omega}=-\log \|\Omega(z)\|^{2},
$$

where $\Omega(z)$ is the $(D, 0)$-form on $Y_{D}$ spanning $H^{D, 0}$ and varies holomorphically in the $z^{I}$. This metric can be explicitly evaluated by introducing a basis of $(D-1,1)$-forms as derivatives of $\Omega$. These are defined by evaluating $\partial_{z^{I}} \Omega$ and projecting the result to the $(D-1,1)$ part, $\left[\partial_{z^{I}} \Omega\right]^{(D-1,1)} \equiv \nabla_{i} \Omega$. We have denoted by $\nabla_{i} \Omega$ the resulting $(D-1,1)$ forms. ${ }^{9}$ Taking derivatives of (2.37) one thus derives the Weil-Petersson metric

$$
g_{I \bar{J}}^{\mathrm{WP}}=\frac{\int_{Y_{D}} \nabla_{I} \Omega \wedge \bar{\nabla} \Omega}{\int_{Y_{D}} \Omega \wedge \bar{\Omega}} .
$$

Let us now give a first, rough, evaluation of this metric near the boundary of the moduli space by using the expansions introduced in section 2.2. For simplicity we will only focus on a single coordinate $t=x+i y$ in the regime $y \gg 1$ and drop all $\zeta^{\kappa}$-dependence labelling the position on the boundary. Inserting (2.18) into the Kähler potential (2.37) we compute the line element

$$
d s_{\mathrm{WP}}^{2}=\frac{1}{y^{2}}(\hat{d}+\gamma(y))\left(d y^{2}+d x^{2}\right),
$$

where $\gamma(y)$ vanishes as $y \rightarrow \infty$. The integer $\hat{d} \in\{0, \ldots, D\}$ is the highest power of $N^{-}$ that does not annihilate $a_{0}$ introduced in (2.18), i.e. $\left(N^{-}\right)^{\hat{d}} a_{0} \neq 0$ while $\left(N^{-}\right)^{\hat{d}+1} a_{0}=0$. Therefore, as long as $\hat{d}>0$, i.e. $N^{-} a_{0} \neq 0$ the metric asymptotes to the Poincaré metric, which is also describing a patch of Euclidian Anti-de Sitter space. Being motivated to establish a holographic perspective one might thus want to either treat the case $\hat{d}=0$ separately, or consider a more suitable metric. In fact, the case $\hat{d}=0$ describes precisely the situation in which the boundary is at finite distance, i.e. that there exists a path to the boundary of finite length in the Weil-Petersson metric. In light of the distance conjecture [2], which discusses infinite distance boundaries, we thus indeed expect that the finite distance case $\hat{d}=0$ is special. The details on how the distinction of the cases $\hat{d}=0$ and $\hat{d}>0$ plays out in studying the states relevant to the distance conjecture was explained in detail in $[3,6,7]$. From the perspective of the underlying structure, however, the case $\hat{d}=0$ is not particularly special as we will see by looking at another well-known metric, the Hodge metric on $\mathcal{M}$, in which this distinction disappears.

While the Weil-Petersson metric is directly physically relevant in Calabi-Yau compactifications, the the so-called Hodge metric $g^{\mathrm{H}}$ is central in Hodge theory. Its properties have been studied in numerous mathematical works (see e.g. [48-54]). In fact, it was also used in the physical study of Calabi-Yau compactifications with background fluxes in [52, 53]. Crucially, it turns out to have 'nicer' properties when considering its curvature tensors, which is mainly due to its universal asymptotic behaviour. The Hodge metric is defined by

$$
g_{I \bar{J}}^{\mathrm{H}}=\sum_{p=0}^{D} \mathcal{G}^{\alpha_{p} \bar{\beta}_{p}} \int_{Y_{D}} \nabla_{I} \chi_{\alpha_{p}} \wedge * \overline{\nabla_{J} \chi_{\beta_{p}}},
$$

\footnotetext{
${ }^{9}$ It can be checked that $\nabla_{i}$ is the Kähler-covariant derivative in this case.
} 
where $\chi_{\alpha_{p}}$ is a basis of $(D-p, p)$-forms and $\nabla_{I} \chi_{\alpha_{p}}$ is the derivative $\partial_{z^{I}} \chi_{\alpha_{p}}$ projected onto the $(D-p-1, p+1)$-component. The matrix $G^{\alpha_{p} \bar{\alpha}_{p}}$ is the inverse of the metric

$$
\mathcal{G}_{\alpha_{p} \bar{\alpha}_{p}}=\int_{Y_{D}} \chi_{\alpha_{p}} \wedge * \overline{\chi_{\alpha_{p}}}
$$

Note that the sum defining $g_{I \bar{J}}^{\mathrm{H}}$ contains terms that are equal to $g_{I \bar{J}}^{\mathrm{WP}}$. In fact, one immediately sees that the summand $p=0$ in (2.40) is exactly the Weil Peterson metric (2.38), since the only $(D, 0)$ is $\Omega$ and the metric (2.41) yields a single term proportional to $\int \Omega \wedge \bar{\Omega}$ in this direction. In addition, the metric $g_{I \bar{J}}^{\mathrm{WP}}$ also appears from the summand $p=D$, since in this case one finds only the $(0, D)$-form $\bar{\Omega}$. This leads us to conclude that $g_{I \bar{J}}^{\mathrm{H}}=2 g_{I \bar{J}}^{\mathrm{WP}}+\ldots$, with the omitted terms being positive definite expressions in the curvature tensors of $g_{I \bar{J}}^{\mathrm{WP}}$. A direct computation reveals that one can explicitly relate the Weil-Petersson and Hodge metric for Calabi-Yau manifolds of arbitrary dimension $D$. For example, it was found in $[50,55,56]$ that

$$
\begin{array}{ll}
D=3: & g_{I \bar{J}}^{\mathrm{H}}=\left(h^{2,1}+3\right) g_{I \bar{J}}^{\mathrm{WP}}+R_{I \bar{J}}^{\mathrm{WP}} \\
D=4: & g_{I \bar{J}}^{\mathrm{H}}=2\left(h^{3,1}+2\right) g_{I \bar{J}}^{\mathrm{WP}}+2 R_{I \bar{J}}^{\mathrm{WP}}
\end{array}
$$

where $R_{I \bar{J}}^{\mathrm{WP}}$ is the Ricci tensor computed in the Weil-Peterson metric. A key observation is that the metric $g_{I \bar{J}}^{\mathrm{H}}$ has a nice asymptotic behaviour. In fact, we will see that its asymptotic form always splits off a part that is a Poincaré metric as long as one has $N_{i}^{-} \neq 0$, for at least one $N_{i}$.

Near the boundary of the moduli space we can use the nilpotent orbit to derive the metric $g_{I \bar{J}}$. Let us, as above, denote by $\chi_{\alpha_{p}}$ a basis of $(D-p, p)$-forms, which are now in the decomposition (2.13), and denote by $\nabla_{I} \chi_{\alpha_{p}}$ the $z^{I}$-derivative projected to the $(D-p-1, p+1)$-piece. We now use the notation (2.15) to write the metric (2.40) as

$$
g_{I \bar{J}}=\sum_{p=0}^{D}\left\langle\chi_{\alpha_{p}} \mid \chi_{\beta_{p}}\right\rangle_{\mathrm{pol}}^{-1}\left\langle\nabla_{I} \chi_{\alpha_{p}} \mid \nabla_{J} \chi_{\beta_{p}}\right\rangle_{\mathrm{pol}},
$$

where we have used the notation established in (2.15).

To give a first study the asymptotic behaviour of the Hodge metric $g_{I \bar{J}}^{\mathrm{H}}$ we again focus on a single coordinate $t=x+i y$ in the regime $y \gg 1$ and drop all $\zeta^{\kappa}$-dependence. To evaluate the leading metric we use (2.18) and its successive derivatives with respect to $t$. The line element for the metric $g_{t \bar{t}}$ now takes the form

$$
d s_{\mathrm{H}}^{2}=\frac{1}{y^{2}}\left(c^{(0)}+\hat{\gamma}(y)\right)\left(d y^{2}+d x^{2}\right)
$$

where now $c^{(0)}=\frac{1}{12} \sum_{i} d_{i}\left(d_{i}+1\right)\left(d_{i}+2\right)$ with a sum over the irreducible $\mathfrak{s l}(2, \mathbb{R})$-representations with highest weight $d_{i}$ in the boundary theory introduced in section 4 , see [57] for details. Crucially, we realize that as long as $N^{-} \neq 0$ we have $c^{(0)}>0$. Note that this implies that the metric indeed becomes the Poincaré metric $\frac{c^{(0)}}{y^{2}}\left(d y^{2}+d x^{2}\right)$ in the limit $y \gg 1$. It is not hard to check that this metric admits an $S l(2, \mathbb{R})$ isometry group. Furthermore, recall that $S l(2, \mathbb{R}) \cong S O(2,1)$ is the global conformal group in one dimension. It might therefore 
be tempting to associate a conformal quantum mechanical system to this setting. In fact, we will see in section 4 that there is indeed a type of 'boundary theory' associated to each asymptotic limit. In fact, we will see that the asymptotic metric (2.44) admits an expansion

$$
d s_{\mathrm{H}}^{2}=\frac{1}{y^{2}}\left(c^{(0)}+\frac{c^{(1)}}{y}+\frac{c^{(2)}}{y^{2}}+\ldots\right)\left(d y^{2}+d x^{2}\right),
$$

and find that the coefficients $c^{(i)}$ are determined non-trivially by a set of boundary data. This strategy can also be applied to obtain the expansion of the Weil-Petersson metric. In the case of having an infinite distance boundary the 'boundary theory' fixes the the asymptotic expansion in powers of $1 / y$ at $y \rightarrow \infty$. Note, however, that in contrast to standard $\mathrm{AdS}_{2}$ holography, we are not considering the conformal boundary at $y \rightarrow 0$.

\section{The bulk theory on the moduli space}

In this section we discuss aspects of the classical bulk theory living on the moduli space. The aim is to find field equations and an action principle for a metric and a matrix-valued field, such that the classical solutions include the 'physical' nilpotent orbits (2.12). In other words, we use the statements of Hodge theory reviewed in section 2 and the existence of a nilpotent orbit as a motivation for a bulk theory. It will then become clear that the field equations of this theory only yield back a nilpotent orbit, if certain boundary conditions are imposed. These are provided by the boundary theory introduced in section 4 . One of the goals of our construction is to capture the information about the asymptotic geometry of moduli spaces without reference to Hodge theory. As noted before, there is a natural action principle associated to one-parameter nilpotent orbits [28]. Recently, a significant generalization has been discussed in [30]. Cecotti also suggested in [31] that an Einstein-Hilbert term with negative cosmological constant can be coupled. Since such a coupling is trivial in two-dimensions, i.e. in the settings most relevant to this work, we suggest in section 3.2 to deviate from [30]. It should be stressed, however, that many aspects of the argument in section 3.1 and 3.2 are similar to [30,31] and have been observed independently as part of this project. The fact that there is a bulk gravity action on moduli space fits rather naturally to the holographic perspective suggested here.

\subsection{Bulk action for matter fields}

In order to construct a bulk theory compatible with the Hodge theory analysis of section 2, we first discuss the relevant field equations without reference to a nilpotent orbit. We then aim to find an action on the moduli space encoding these equations. We will focus on a real two-dimensional moduli space and later comment on possible higher-dimensional generalizations.

Instead of fixing $\mathcal{M}$ to be the moduli space of a Calabi-Yau manifold, we consider $\mathcal{M}$ abstractly as being the real two-dimensional world-sheet of a bulk sigma model. We denote the local world-sheet coordinates by $\sigma^{1}, \sigma^{2}$. This sigma-model has matter fields $\hat{h}(\sigma)$ that take values in the group $G_{\mathbb{R}}$, which is the target space of the bulk sigma model. The group $G_{\mathbb{R}}$ acts on a finite-dimensional complex Hilbert space $\mathcal{H}$ and preserves some bilinear form 
$\langle v, w\rangle$. We denote the fixed inner product on $\mathcal{H}$ by $\langle v \mid w\rangle_{\text {ref. }}$ A non-trivial requirement is that the inner product can be written using a grading element $Q \in i \mathfrak{g}_{\mathbb{R}}$, which we call the charge operator. By definition, this operator is a semisimple algebra element which splits $\mathfrak{g}_{\mathbb{C}}$ into eigenspaces with integer eigenvalues and obeys $\bar{Q}=-Q$. Given any operator $\mathcal{O} \in \mathfrak{g}_{\mathbb{C}}$, we can then make a decomposition $\mathcal{O}=\sum_{l} \mathcal{O}_{l}$ such that

$$
\left[Q, \mathcal{O}_{l}\right]=l \mathcal{O}_{l}, \quad l=-D, \ldots, D
$$

This grading element gives an essentially equivalent way to formulate a Hodge decomposition and their infinitesimal variations. ${ }^{10}$ Motivated by the Hodge theory construction we now require as in section 2 that

$$
\langle v \mid w\rangle_{\mathrm{ref}}=\left\langle v, i^{2 Q} w\right\rangle .
$$

Note that we infer by using (2.4) that $Q^{\dagger}=Q$, with the adjoint taken with respect to $\langle v \mid w\rangle_{\text {ref. }}$.

Given this structure we can now discuss the field equations for the matter fields $\hat{h}$. Let us first restate the second equation in (2.29) using $\hat{h}$, which amount to writing

$$
2 \partial_{y}\left(\hat{h}^{-1} \partial_{y} \hat{h}\right)=\left[\left(\hat{h}^{-1} \partial_{x} \hat{h}\right)^{\dagger}, \hat{h}^{-1} \partial_{x} \hat{h}\right] .
$$

Furthermore, we recall that the equations (2.30) can be rewritten using $\hat{h}$ and take the form

$$
-2\left[Q, \hat{h}^{-1} \partial_{y} \hat{h}\right]=i\left(\left(\hat{h}^{-1} \partial_{x} \hat{h}\right)^{\dagger}+\hat{h}^{-1} \partial_{x} \hat{h}\right), \quad\left[Q, \hat{h}^{-1} \partial_{x} \hat{h}\right]=i \hat{h}^{-1} \partial_{y} \hat{h} .
$$

We note that this equation implies

$$
\left(\hat{h}^{-1} \partial_{y} \hat{h}\right)^{\dagger}=\hat{h}^{-1} \partial_{y} \hat{h} .
$$

Clearly, the equations (3.3), (3.4) are not democratic in $x, y$, which is due to the fact that in the nilpotent orbit solutions the coordinate $x$ appears only through the exponential $e^{x N^{-}}$ as seen in (2.23). This simple dependence yields the conditions

$$
\partial_{x}\left(\hat{h}^{-1} \partial_{y} \hat{h}\right)=\partial_{x}\left(\hat{h}^{-1} \partial_{x} \hat{h}\right)=0 .
$$

We can also realize these constraints by requiring the existence of a continuous symmetry

$$
\hat{h}(x+c, y)=e^{c N^{-}} \hat{h}(x, y),
$$

where $c$ is any real constant and $N^{-}$is some real matrix in $\mathfrak{g}_{\mathbb{R}}$. To obtain an unconstrained set of equations of motion democratic in $\sigma^{1} \equiv x, \sigma^{2} \equiv y$ we can combine (3.6) with (3.3) and (3.5) to obtain

$$
\sum_{\alpha} \partial_{\sigma^{\alpha}}\left(\hat{h}^{-1} \partial_{\sigma^{\alpha}} \hat{h}+\left(\hat{h}^{-1} \partial_{\sigma^{\alpha}} \hat{h}\right)^{\dagger}\right)-\left[\left(\hat{h}^{-1} \partial_{\sigma^{\alpha}} \hat{h}\right)^{\dagger}, \hat{h}^{-1} \partial_{\sigma^{\alpha}} \hat{h}\right]=0 .
$$

\footnotetext{
${ }^{10}$ See $[46,47]$ for the mathematical details.
} 
In the following we will view these equations as equations of motion for $\hat{h}$. That this is plausible can be further motivated in various ways. Firstly, we could have considered in section 2 the full variation of Hodge structure as done in [30]. Secondly, we can obtain the equations of motion (3.8) from an action that arises as natural generalization of (2.32) as we will see in the following.

To obtain a bulk matter action principle, we start with the one-dimensional action (2.32). To obtain a two-dimensional action for the matter fields $\hat{h}(\sigma)$, we first replace $h^{-1} \partial_{y} h=$ $\hat{h}^{-1} \partial_{y} \hat{h}$ and $h^{-1} N^{-} h=\hat{h}^{-1} \partial_{x} \hat{h}$ by temporarily assuming the simple $x$-dependence $\hat{h}=$ $e^{x N^{-}} h$. Integrating over $\sigma^{1} \equiv x$ the action (2.32) then generalizes to an action $S(\hat{h})$ as

$$
S(\hat{h})=\frac{1}{2} \int d^{2} \sigma\left(\operatorname{Tr}\left|\left(\hat{h}^{-1} \partial_{\sigma^{2}} \hat{h}\right)^{\dagger}+\hat{h}^{-1} \partial_{\sigma^{2}} \hat{h}\right|^{2}+2 \operatorname{Tr}\left|\hat{h}^{-1} \partial_{\sigma^{1}} \hat{h}\right|^{2}\right),
$$

where $|A|^{2}=A^{\dagger} A$ and $d^{2} \sigma=d \sigma^{1} d \sigma^{2}$. We can check that adding the terms $\operatorname{Tr}\left(\hat{h}^{-1} \partial_{\sigma^{1}} \hat{h}\right)^{2}$ and $\operatorname{Tr}\left(\left(\hat{h}^{-1} \partial_{\sigma^{1}} \hat{h}\right)^{\dagger}\right)^{2}$ to the action does not change the equations of motion. This allows us to write

$$
S_{\text {mat }}(\hat{h})=\frac{1}{2} \int d^{2} \sigma \sum_{\alpha} \operatorname{Tr}\left|\left(\hat{h}^{-1} \partial_{\sigma^{\alpha}} \hat{h}\right)^{\dagger}+\hat{h}^{-1} \partial_{\sigma^{\alpha}} \hat{h}\right|^{2}
$$

That the equations of motion resulting from this action are indeed given by (3.8) can be checked by using

$$
\delta\left(\hat{h}^{-1} \partial_{\sigma^{\alpha}} \hat{h}\right)=-\left[\hat{h}^{-1} \delta \hat{h}, \hat{h}^{-1} \partial_{\sigma^{\alpha}} \hat{h}\right]+\frac{d}{d \sigma^{\alpha}}\left(\hat{h}^{-1} \delta \hat{h}\right) .
$$

Let us note that the action (3.10) agrees with the one found in [30], which was shown to yield the $t t^{*}$ equations. We also see that the generalization to higher-dimensional field spaces of (3.10) and (3.4) appears to be straightforward. This is partly deceiving, since further constraints arise from imposing commutativity of certain derivatives $\hat{h}^{-1} \partial_{\sigma^{\alpha}} \hat{h}$. When looking at near boundary solutions a very non-trivial structure emerges [27] that we will not further analyze in this work. Our study of solutions to (3.8) and (3.4) near the boundary does apply, however, to higher-dimensional moduli spaces where $\hat{h}$ depends on two coordinates $\sigma^{1}, \sigma^{2}$ near the boundary and a number of directions that remain in the bulk and are suppressed in the notation.

It is important to note that the action (3.10) has to be supplemented by (3.4). From the above discussion it should be clear that the existence of $Q$ and the constraint (3.4) is central to the construction and it would be desirable to find an action principle that also yields the latter constraint. In the bulk-boundary matching of section 5 , however, we will only need the field equations (3.3) and (3.4) and therefore leave the construction of a complete action principle to future work. Furthermore, it is crucial to impose a set of boundary conditions to obtain solutions to these field equations that correspond to nilpotent orbits as we will see in section 5. Firstly, one has to require the symmetry (3.7) which constrains the $x$-dependence of $\hat{h}$. Secondly, note that a solution to (3.3) with (3.4) gives also a solution to Nahm's equations (2.29) when setting

$$
\mathcal{N}^{0}:=-2 \hat{h}^{-1} \partial_{y} \hat{h}, \quad \mathcal{N}^{-}:=\hat{h}^{-1} \partial_{x} \hat{h}, \quad \mathcal{N}^{+}:=\left(\mathcal{N}^{-}(y)\right)^{\dagger} .
$$


The solutions to Nahm's equations give rise at the poles of $\mathcal{N}^{0}, \mathcal{N}^{ \pm}$to a special triples of operators that commute as generators of $\mathfrak{s l}(2, \mathbb{R}) \cong \mathfrak{s u}(2)$ [58]. In the situation at hand, we are interested in solutions near $y=\infty$ and we will consider general bulk solutions of the form

$$
\mathcal{N}^{0}=\frac{\tilde{N}^{0}}{y}+\mathcal{O}\left(y^{-\frac{3}{2}}\right), \quad \mathcal{N}^{ \pm}=\frac{\tilde{N}^{ \pm}}{y}+\mathcal{O}\left(y^{-\frac{3}{2}}\right)
$$

The coefficients of the slowest decreasing term of $\mathcal{N}^{0}, \mathcal{N}^{ \pm}$are triples $\left(\tilde{N}^{0}, \tilde{N}^{ \pm}\right)$, which are generators of an algebra $\mathfrak{s l}(2, \mathbb{R})$ as we will discuss below. One can think of the $\left(\tilde{N}^{0}, \tilde{N}^{ \pm}\right)$as setting part of the boundary conditions for the solution $\mathcal{N}^{0}, \mathcal{N}^{ \pm}$. Furthermore, to implement the positivity of the norms over the moduli space, we also need to make sure that there is a well-defined reference structure $|v\rangle_{\text {ref }}$, which we will argue should exist on the boundary. Therefore, to single out solutions that correspond to physical situations, i.e. to a valid $F_{\mathrm{pol}}^{p}$, we will then turn this into a requirement for a specification of the physical boundary conditions. In the next section 4 we suggest that these boundary conditions arise from a certain 'boundary theory'.

\subsection{On the coupling to gravity}

In the following we would like to briefly comment on the possibility to couple the matter action of section 3.1 to gravity. As before, we will mostly focus on a one-dimensional moduli space, but later comment on possible generalizations. Several aspects of the following discussion have recently also appeared in [31]. We will make contact with the results given there and highlight where our construction differs.

From a holographic perspective it would be natural if there is actually a gravity theory on the moduli space with a dynamical metric $g_{\alpha \beta}$. For a real two-dimensional $\mathcal{M}$, however, we recall that Einstein gravity is non-dynamical and the Einstein-Hilbert term reduces to a constant. In fact, we can think of (3.10) as a two-dimensional string world-sheet action gauge-fixed to the trivial metric $\delta_{\alpha \beta}$. The coupling to a general metric $g_{\alpha \beta}$ yields then the action

$$
S_{\text {mat }}(g, \hat{h})=\int_{\mathcal{M}} d^{2} \sigma \sqrt{g} \mathcal{L}_{\text {mat }},
$$

with

$$
\left.\left.\mathcal{L}_{\text {mat }}=\frac{1}{2} g^{\alpha \beta} \operatorname{Tr}\left[\left(\hat{h}^{-1} \partial_{\sigma^{\alpha}} \hat{h}\right)^{\dagger}+\hat{h}^{-1} \partial_{\sigma^{\alpha}} \hat{h}\right]\right]\left[\left(\hat{h}^{-1} \partial_{\sigma^{\beta}} \hat{h}\right)^{\dagger}+\hat{h}^{-1} \partial_{\sigma^{\beta}} \hat{h}\right]\right] .
$$

There are now two sets of equations of motion: (1) the equations of motion of $\hat{h}$, and (2) the equations of motion for $g_{\alpha \beta}$. The latter simply correspond to the statement that the energy-momentum tensor vanishes,

$$
T_{\alpha \beta}^{\operatorname{mat}}=0 .
$$

Let us now compute $T_{\alpha \beta}^{\text {mat }}$ and check when it vanishes on-shell for a nilpotent orbit solution $\hat{h}$.

In evaluating the energy momentum tensor $T_{\alpha \beta}^{\text {mat }}$ we use the important observation that in complex coordinates $t=\sigma^{1}+i \sigma^{2}$ one finds that the integrand in (3.10) can be expressed by using the Hodge metric (2.43) when evaluated on a solution $\hat{h}$ corresponding to a nilpotent orbit. To see this, we use a basis of $(D-p, p)$-forms $\hat{h}|v\rangle_{\text {ref }}$ in the expression for 
the Hodge metric (2.43). If $\hat{h}|v\rangle_{\text {ref }}$ is of type $(D-p, p)$ then also $|v\rangle_{\text {ref }}$ is of type $(D-p, p)$, but now in the reference Hodge decomposition (2.23). Since $\mathcal{L}_{-1}$ obeys (2.35) we conclude that $\mathcal{L}_{-1}|v\rangle_{\text {ref }}$ is a $(D-p-1, p+1)$-form as can be checked by recalling (2.24). Hence, we realize that $\mathcal{L}_{-1}$ acts as $\nabla_{t}$ appearing in the Hodge metric (2.43) and allows us to rewrite the line element of $g^{\mathrm{H}}$. Concretely, using $\operatorname{Tr}\left(\mathcal{L}_{-1}^{2}\right)=-\operatorname{Tr}\left(\left[Q, \mathcal{L}_{-1}\right] \mathcal{L}_{-1}\right)=0$, the line element of the Hodge metric can be written as

$$
\begin{aligned}
g_{t \bar{t}}^{\mathrm{H}}(\hat{h}) d t d \bar{t} & =\operatorname{Tr}\left|\mathcal{L}_{-1} d t\right|^{2}=\frac{1}{2} \operatorname{Tr}\left|\mathcal{N}^{+}+\mathcal{N}^{-}\right|^{2}\left(d \sigma^{1}\right)^{2}+\frac{1}{2} \operatorname{Tr}\left|\mathcal{N}^{0}\right|^{2}\left(d \sigma^{2}\right)^{2} \\
& =\frac{1}{2} \sum_{\alpha} \operatorname{Tr}\left|\left(\hat{h}^{-1} \partial_{\sigma^{\alpha}} \hat{h}\right)^{\dagger}+\hat{h}^{-1} \partial_{\sigma^{\alpha}} \hat{h}\right|^{2}\left(d \sigma^{\alpha}\right)^{2}
\end{aligned}
$$

where we have used (2.34) in the second equality and (2.31), (3.12) in the third. Therefore, the Lagrangian density $\mathcal{L}_{\text {mat }}$ and the energy momentum tensor $T_{\alpha \beta}^{\text {mat }}$ can be evaluated on a nilpotent orbit solution as

$$
\mathcal{L}_{\text {mat }}=\frac{1}{2} g^{\alpha \beta} g_{\alpha \beta}^{\mathrm{H}}, \quad T_{\alpha \beta}^{\mathrm{mat}}=g_{\alpha \beta}^{\mathrm{H}}-\frac{1}{2} g_{\alpha \beta} g^{\gamma \delta} g_{\gamma \delta}^{\mathrm{H}},
$$

where we have expressed $g_{\alpha \beta}^{\mathrm{H}}$ in real coordinates, keeping in mind that this metric is Kähler and hence obeys $g_{t t}^{\mathrm{H}}=g_{\bar{t} t}^{\mathrm{H}}=0$. It is now easy to see that $T_{\alpha \beta}^{\mathrm{mat}}=0$ for any choice of metric $g_{\alpha \beta}$ on $\mathcal{M}$ that satisfies $g_{t t}=g_{\overline{t t}}=0$. In particular, we can consider $g_{\alpha \beta}$ to be the Kähler metrics $g_{\alpha \beta}=g_{\alpha \beta}^{\mathrm{WP}}$ or $g_{\alpha \beta}=g_{\alpha \beta}^{\mathrm{H}}$ and satisfy the complete set of equations of motion of (3.14). The latter fact was also shown in general dimension in [30, 31].

While this result is encouraging it appears that one should look for a more sophisticated two-dimensional gravity theory. Ideally one would like to construct a two-dimensional theory, such that $g_{t \bar{t}}^{\mathrm{WP}}$ with the expansion (2.39) is a solution at infinite distance points. Starting again with the nilpotent orbit solutions for the matter theory and imposing (3.16), we realize from the above discussion that there is only a single real function, namely $g_{t \bar{t}}$, in the metric $g_{\alpha \beta}$ to be fixed. This can be done by imposing one real equation relating the matter part of the theory to the pure gravity part. In particular, we will now check that imposing

$$
R-2 \Lambda=2 \kappa^{2} \mathcal{L}_{\text {mat }}
$$

does indeed ensure that $g_{t \bar{t}}=g_{t \bar{t}}^{\mathrm{WP}}$, if we fix $\Lambda$ and $\kappa$ appropriately. Considering $\mathcal{L}_{\text {mat }}$ on a nilpotent orbit solution $\hat{h}$, we use (3.18) to show that (3.19) can be written as

$$
g^{\alpha \beta}\left(R_{\alpha \beta}-4 \Lambda g_{\alpha \beta}\right)=\kappa^{2} g^{\alpha \beta} g_{\alpha \beta}^{\mathrm{H}} .
$$

The real equation fixes $g_{t \bar{t}}$ in case we impose $T_{\alpha \beta}^{\text {mat }}=0$. To ensure the match with $g_{t \bar{t}}=g_{t \bar{t}}^{\mathrm{WP}}$, we now fix $\kappa, \Lambda$ by assuming $g_{\alpha \beta}=g_{\alpha \beta}^{\mathrm{WP}}$ and exploiting the relation of the Hodge metric $g_{\alpha \beta}^{\mathrm{H}}$ with $g_{\alpha \beta}^{\mathrm{WP}}$. Recall the that we have given the explicit relations in (2.42) for Calabi-Yau threefolds $(D=3)$ and Calabi-Yau fourfolds $(D=4)$ and refer to [53] for the general discussion. For these two cases one finds

$$
D=3: \quad \Lambda=-1, \kappa^{2}=1, \quad D=4: \quad \Lambda=-\frac{3}{4}, \kappa^{2}=\frac{1}{2} .
$$


It would be desirable to formulate an action principle imposing the field equations (3.4), (3.8), (3.16), (3.19) on $\hat{h}$ and $g_{\alpha \beta}$. A natural choice appears to be the coupling of (3.14) to the Jackiw-Teitelboim gravity action [59,60]. Introducing an auxiliary field $\Phi(\sigma)$ the full action then reads

$$
S(\hat{h}, g, \Phi)=-\frac{1}{2 \kappa^{2}} \int_{\mathcal{M}} d^{2} \sigma \sqrt{g} \Phi(R-2 \Lambda)+\int_{\mathcal{M}} d^{2} \sigma \sqrt{g} \Phi \mathcal{L}_{\text {mat }}
$$

The field $\Phi$ acts as a Lagrange multiplier and enforces the condition (3.19). We stress, however, that this theory is likely not complete and deserves further study. In particular, we find that the coupling to $\Phi$ also modifies the matter equations of motion and we recall that we need to impose the constraint (3.4) on the matter fields. Furthermore, we have not discussed possible boundary terms in the action (3.22). It would be desirable to identify the correct gravity model, if it exists at all, from the plethora of two-dimensional possibilities [61, 62].

Let us close by noting that for higher-dimensional moduli spaces a coupling to gravity with a Einstein-Hilbert action with a cosmological constant is possible [31]. Also in these cases one can determine $\kappa$ and $\Lambda$ depending on $D$ and the dimensionality of $\mathcal{M}$, such that the 'physical' metric $g_{\alpha \beta}^{\mathrm{WP}}$ is a solution to the Einstein equations. In light of the above observations and with a focus on a holographic perspective it would be nice to see if this is indeed the correct coupling to gravity. In the remainder of the paper we will mostly restrict to the two-dimensional situation and, since we are only talking about classical aspects of the theory, it will suffice to work with the field equations (3.8) and (3.4) directly.

\section{The $\mathrm{Sl}(2, \mathbb{C})$ boundary theory}

In this section we discuss the structure that arises at the boundary $\partial \mathcal{M}$ of the moduli space $\mathcal{M}$ with a focus on a $\operatorname{dim} \mathcal{M}-1$ dimensional component. Recalling that the boundary splits into multiple such components $\Delta_{k}$, as discussed around (2.9), we thus want to describe the boundary theory for a fixed component, say $\Delta_{0}$, and describe how in a local patch around it the Hodge norm and the Hodge decomposition can be determined in the bulk. We introduce local coordinates $z \equiv e^{2 \pi i t}$ and $\zeta^{\kappa}$, such that the boundary component is approached in the limit

$$
z \rightarrow 0 \quad \text { or } \quad t=x+i y \rightarrow x_{0}+i \infty .
$$

Suppressing the $\zeta^{\kappa}$ coordinate directions, the considered configuration can be depicted as in figure 2. The cases of higher co-dimension is significantly more involved and goes beyond the scope of this work.

Let us begin by briefly motivating how the relevant data on the boundary is extracted. As for the bulk theory the motivation comes from Hodge theory, or more precisely the existence of a nilpotent orbit introduced in section 2.2. The non-trivial task is then to extract the relevant data in the limit $y \rightarrow \infty$. Roughly stated, one performs a clever expansion of $F_{\mathrm{pol}}^{p}$ in $t$ around the point $y=\infty$ and analyses the information carried by the various terms near the boundary of $\mathcal{M}$. One then finds that an intriguing algebraic 
structure with $\mathfrak{s l}(2, \mathbb{C})$ symmetry emerges and we propose that this should be the symmetry algebra of a boundary theory. To understand how the nilpotent orbit defines the boundary data the reader may later consult section 5 where we match the bulk and boundary theories. Given a nilpotent orbit the boundary data can always be extracted as we will describe in section 5.3.

In this section we describe the for us relevant structures of the boundary theory abstractly without describing how the data is derived from Hodge theory. More precisely, we will discuss the following boundary data in detail:

(1) Hilbert space:

(2) boundary charge decomposition:

$(3) \mathfrak{s l}(2, \mathbb{C})$-algebra in $\mathfrak{g}$ :

(4) phase operator in $\mathfrak{g}$ :

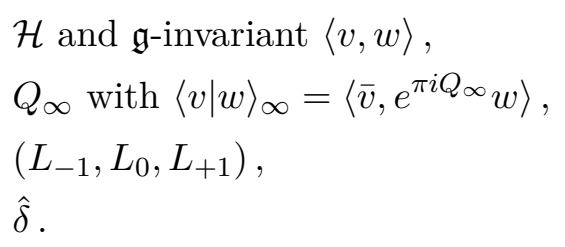

Our discussion will make very little reference to the underlying Hodge theory and will be formulated using operators rather than $(p, q)$-splittings. ${ }^{11}$ It is important to stress that we will not attempt to construct the full boundary theory, but rather note that the data (4.2) is sufficient for the purposes of this work. We will will sometimes refer loosely to the data (4.2) as being the 'boundary theory'.

As an aside, let us not that an actual boundary theory could be obtained by using the data (4.2) to define a conformal quantum mechanics model. To introduce a time dependence, we could consider the boundary to still depend on a coordinate $\tau=\frac{1}{2 \pi i} \log x_{0}$ that is unaffected when taking the limit (4.1) and view $\tau$ as Euclidean time. On the quotient space with coordinates $z$, we have $\tau \cong \tau+1$. One might want to visualize this cutting out the singularity of the disk (a.2) in figure 2. Working on the disk we can build local operators $\mathcal{O}_{q}^{[d]}(\tau)$ of charge $q$ and weight $d$ by setting

$$
\mathcal{O}_{q}^{[d]}(\tau)=\sum_{l=-d}^{d} e^{2 \pi i l \tau} \mathcal{O}_{q}^{(d, l)},
$$

where $q, l, d$ are the eigenvalues under $Q_{\infty}, L_{0}$, and the $\mathfrak{s l}(2, \mathbb{C})$-Casimir, respectively. This decomposition of operators will be discussed in detail in section 4.3 below. As for the quantum mechanical model with $\mathfrak{s l}(2, \mathbb{R})$-symmetry suggested in [63], it is not immediate how to interpret this theory as a $\mathrm{CFT}_{1}$. There is no distinguished vacuum state and the formulation of a state-operator-map is obscured. Interestingly, both issues have been addressed in [64] for the model of [63]. The proposed solutions appear to be equally important in our setting. Despite these similarities we believe that the actual boundary theory should be more involved and we hope to address a detailed formulation in the future.

\subsection{Boundary charge operator and $\mathfrak{s l}(2, \mathbb{C})$-algebra}

To start with we note that this boundary theory consists of a finite dimensional Hilbert space of states $\mathcal{H}$. This Hilbert space is obtained by the complexification of a lattice $\mathcal{L}$

\footnotetext{
${ }^{11}$ In the mathematics literature such an approach was given by Robles [46].
} 
as $\mathcal{H}=\mathbb{C} \otimes \mathcal{L}$. Note that as a vector space $\mathcal{H}$ is isomorphic to the space introduced in section 3. We will also consider its real version $\mathcal{H}_{\mathbb{R}}=\mathbb{R} \otimes \mathcal{L}$. In geometric settings, such as in Calabi-Yau compactifications, we identify $\mathcal{L}=H^{D}\left(Y_{D}, \mathbb{Z}\right), \mathcal{H}=H^{D}\left(Y_{D}, \mathbb{C}\right)$, and $\mathcal{H}_{\mathbb{R}}=H^{D}\left(Y_{D}, \mathbb{R}\right) .{ }^{12}$ We require that the norm of $\mathcal{H}$ is induced by a charge operator $Q_{\infty}$ and that it is compatible with an $\mathfrak{s l}(2, \mathbb{C})$-algebra. We will introduce these objects in the following.

The charge operator $\mathbf{Q}_{\infty}$ and inner product. The charge operator $Q_{\infty}$ can be viewed as defining a boundary Hodge decomposition

$$
\mathcal{H}=H_{\infty}^{D, 0} \oplus H_{\infty}^{D-1,1} \oplus \ldots \oplus H_{\infty}^{0, D},
$$

where $H_{\infty}^{q, p}=\overline{H_{\infty}^{p, q}}$ and $p+q=D .{ }^{13}$ The decomposition (4.4) can equally be encoded by specifying a charge operator $Q_{\infty} \in \mathfrak{g}_{\mathbb{C}}$ acting as

$$
Q_{\infty}|w\rangle=\frac{1}{2}(2 p-D)|w\rangle \quad \text { for } \quad|w\rangle \in H_{\infty}^{p, D-p}
$$

where we are using a bra-ket notation for states. Note that one infers from the properties of (4.4) that

$$
\bar{Q}_{\infty}=-Q_{\infty} .
$$

We also require that the decomposition (4.4) allows us to define a boundary Hodge operator $C_{\infty}$ by setting

$$
C_{\infty}|w\rangle=i^{2 p-D}|w\rangle \quad \text { for } \quad|w\rangle \in H_{\infty}^{p, D-p},
$$

which defines a norm $\|\cdot\|_{\infty}$. Formally one can thus relate $C_{\infty}=i^{2 Q_{\infty}}=e^{\pi i Q_{\infty}}$, with $Q_{\infty}$ acting as in (4.5). Concretely, we define the inner product and the norm by

$$
\langle v \mid w\rangle_{\infty}:=\left\langle\bar{v}, C_{\infty} w\right\rangle, \quad\|v\|_{\infty}^{2}=\langle v \mid v\rangle .
$$

It is a non-trivial fact that such a charge operator $Q_{\infty}$ with associated non-degenerate norm exists on every boundary component of a complex structure moduli space [27]. To come to this conclusion the reader can consult section 5 and read the explanation with the assumption that an underlying nilpotent orbit exists.

The boundary algebra $\mathfrak{s l}(\mathbf{2}, \mathbb{R})$. We next introduce an operator algebra representing $\mathfrak{s l}(2, \mathbb{R})$ on $\mathcal{H}$. This can be motivated by the fact that the Hodge metric introduced in section 2.4 always asymptotes to the Poincaré metric, which has an $\mathfrak{s l}(2, \mathbb{R})$ isometry algebra. We thus define real generators $N^{0}, N^{ \pm} \in \mathfrak{g}_{\mathbb{R}}$ that satisfy the angular momentum algebra ${ }^{14}$

$$
\left[N^{0}, N^{ \pm}\right]= \pm 2 N^{ \pm}, \quad\left[N^{+}, N^{-}\right]=N^{0} .
$$

\footnotetext{
${ }^{12}$ More precisely, we would actually have to consider the primitive parts of $H^{D}\left(Y_{D}\right)$.

${ }^{13}$ This decomposition can still vary with the change in the coordinates $\zeta^{\kappa}$ not taken to a limit, but is, of course, independent of $t$.

${ }^{14}$ Note that, interpreting $\mathfrak{s l}(2, \mathbb{R})$ as the global conformal group in one dimensions, one can identify $H=\frac{1}{2} N^{0}$ as the global Hamiltonian, $K=N^{-}$as the special conformal transformations, and $P=-N^{+}$as translations. Below in (4.11) we will see that that with respect to the inner product (4.8) we have $P^{\dagger}=-K$.
} 
We want to ensure that this algebra is compatible with the split (4.4) and hence introduce commutation relations with the charge operator $Q_{\infty}$. This operator is imaginary and hence we are naturally lead to formulate compatibility conditions over the complex numbers. We thus require

$$
\left[Q_{\infty}, N^{0}\right]=i\left(N^{+}+N^{-}\right), \quad\left[Q_{\infty}, N^{ \pm}\right]=-\frac{i}{2} N^{0} .
$$

Since $C_{\infty}=i^{2 Q_{\infty}}$ these compatibility conditions imply that

$$
\left(N^{0}\right)^{\dagger}=N^{0}, \quad\left(N^{+}\right)^{\dagger}=N^{-}
$$

where $\mathcal{O}^{\dagger}=-C_{\infty}^{-1} \overline{\mathcal{O}} C_{\infty}$ is the adjoint operator to $\mathcal{O}$ with respect to the inner product $\langle v \mid w\rangle_{\infty}$ introduced in (4.8). It is interesting to stress that there are at least two major differences to the conformal quantum mechanics model discussed, for example, in [63, 64]. Firstly, we are considering finite-dimensional representations of $\mathfrak{s t}(2, \mathbb{R})$ which are thus not unitary. Secondly, the central importance of the operator $Q_{\infty}$ naturally leads us to consider a complex operator algebra. In fact, we will see next that the boundary symmetry is better described by the algebra $\mathfrak{s l}(2, \mathbb{C})$ rather than $\mathfrak{s l}(2, \mathbb{R})$.

The boundary algebra $\mathfrak{s l}(\mathbf{2}, \mathbb{C})$. Due to the fact that the split $(4.4)$ is over the complex numbers, we are lead to introduce the generators $L_{\alpha} \in \mathfrak{g}_{\mathbb{C}}$ representing an $\mathfrak{s l}(2, \mathbb{C})$ action on $\mathcal{H}$. These are defined in terms of the $N^{0}, N^{ \pm}$as

$$
L_{ \pm 1}:=\frac{1}{2}\left(N^{+}+N^{-} \mp i N^{0}\right), \quad L_{0}:=i\left(N^{-}-N^{+}\right) .
$$

Using (4.9) we see that the $L_{\alpha}$ satisfy the commutation relations

$$
\left[L_{0}, L_{ \pm 1}\right]= \pm 2 L_{ \pm 1}, \quad\left[L_{1}, L_{-1}\right]=L_{0}
$$

Furthermore, we have that

$$
\bar{L}_{0}=-L_{0}, \quad \bar{L}_{1}=L_{-1} .
$$

In addition, we note from (4.10) that the compatibility with the split (4.4) amounts to the commutation relations with $Q_{\infty}$ given by

$$
\left[Q_{\infty}, L_{\alpha}\right]=\alpha L_{\alpha}
$$

which clarifies the meaning of the subscript on $L_{\alpha} \cdot{ }^{15}$ Note that (4.14) together with (4.15) implies that ${ }^{16}$

$$
L_{\alpha}^{\dagger}=L_{-\alpha},
$$

where $L_{\alpha}^{\dagger}$ is the adjoint operator with respect to the inner product $\langle v \mid w\rangle_{\infty}$.

The conditions on $L_{\alpha}$ ensure the compatibility of the $\mathfrak{s l}(2, \mathbb{C})$ representation with the boundary decomposition (4.4). The statement can rephrased by noting the $\mathfrak{s l}(2, \mathbb{C})$ itself

\footnotetext{
${ }^{15}$ In mathematics a triple with $L_{0}$ having even charge and $L_{ \pm 1}$ having odd charge while satisfying (4.13) and (4.14) is also known as Đoković-Konstant-Sekiguchi (DKS) triple and plays an important role in the DKS correspondence.

${ }^{16}$ Recall that $\mathfrak{g}_{\mathbb{C}}$ is the algebra of elements preserving $\langle\cdot, \cdot\rangle$ given in $(2.3)$, e.g. one has $\langle L \alpha, \beta\rangle=-\langle\alpha, L \beta\rangle$.
} 
has a natural charge split with eigenspaces $\mathfrak{s l}(2, \mathbb{C})^{0}, \mathfrak{s l}(2, \mathbb{C})^{1}, \mathfrak{s l}(2, \mathbb{C})^{-1}$ of charge $-1,0,1$, respectively. These charge eigenspaces are the one-dimensional complex spaces spanned by $\mathbf{l}_{0}=i\left(\mathbf{n}^{-}-\mathbf{n}^{+}\right)$, and $\mathbf{l}_{ \pm 1}=\frac{1}{2}\left(\mathbf{n}^{+}+\mathbf{n}^{-} \mp i \mathbf{n}^{z}\right)$, respectively. Here one is using the standard $\mathfrak{s l}(2, \mathbb{R})$ generators

$$
\mathbf{n}^{-}=\left(\begin{array}{ll}
0 & 1 \\
0 & 0
\end{array}\right), \quad \mathbf{n}^{+}=\left(\begin{array}{ll}
0 & 0 \\
1 & 0
\end{array}\right), \quad \mathbf{n}^{z}=\left(\begin{array}{cc}
-1 & 0 \\
0 & 1
\end{array}\right) .
$$

The equation (4.15) then corresponds to the requirement that this split of $\mathfrak{s l}(2, \mathbb{C})$ is represented in the split of $\mathfrak{g}$ induced by $Q_{\infty}$. In fact, it requires that there exists a map $\varphi: S l(2, \mathbb{C}) \rightarrow G_{\mathbb{C}}$ such that $\varphi_{*} \mathbf{l}_{\alpha}=L_{\alpha}$. In mathematical terms such a representation is known as a horizontal $S l(2)$ with respect to the splitting (4.4).

\subsection{Boundary eigenstates}

With the existence of the $\mathfrak{s l}(2, \mathbb{C})$ algebra on the boundary, we can define another canonical splitting of $\mathcal{H}$. This splitting can be performed over the real numbers or complex numbers, i.e. by considering $\mathcal{H}_{\mathbb{R}}$ or $\mathcal{H}$.

The real boundary splitting. For the real space $H^{D}\left(Y_{D}, \mathbb{R}\right)$ we introduce an eigenbasis labelled by two quantum numbers $(l, d)$ for the $\mathfrak{s u}(2)$-algebra (4.9). The label $l \in\{-d, \ldots, d\}$ corresponds to the eigenvalue of $N^{0}$, while $d \in\{0, \ldots, D\}$ is related to the eigenvalues $d(d+2)$ of the Casimir operator

$$
N^{2}=2 N^{+} N^{-}+2 N^{-} N^{+}+\left(N^{0}\right)^{2} .
$$

Denoting the eigenstates by $|d, l\rangle$ we thus have

$$
\begin{aligned}
N^{2}|d, l\rangle & =d(d+2)|d, l\rangle, & d & =0, \ldots, D, \\
N^{0}|d, l\rangle & =l|d, l\rangle, & l & =-d, \ldots, d,
\end{aligned}
$$

and note that there can be many states with the same labels, but we will not distinguish them with an extra index. The set of highest weight states is then given by

$$
\text { highest weight states }|d, d\rangle: \quad N^{+}|d, d\rangle=0,
$$

with all other states being generated by acting with $N^{-}$. We thus have the split

$$
\mathcal{H}_{\mathbb{R}}=\bigoplus_{d} \bigoplus_{l \in\{-d, \ldots, d\}} V_{l}^{d} \equiv \bigoplus_{\ell \in \mathcal{E}} V_{\ell},
$$

where $V_{l}^{d}$ are the real vector spaces spanned by the eigenstates $|d, l\rangle$ introduced in (4.19). We have also introduced the set $\mathcal{E}$ of all possible values $\ell=(d, l)$ in order to simplify the expressions.

The splitting is orthogonal in the boundary inner product introduced in (4.8). Consider $|d, l\rangle \in V_{l}^{d}$ and $\left|d^{\prime}, l^{\prime}\right\rangle \in V_{l^{\prime}}^{d^{\prime}}$. Then one has the identities

$$
\begin{aligned}
\left\langle d, l \mid d^{\prime}, l^{\prime}\right\rangle & =0 \quad \text { for } \quad d \neq d^{\prime}, l \neq l^{\prime}, \\
\langle d, l \mid d, l\rangle & >0 .
\end{aligned}
$$


Note that in general one has several states $|d, l\rangle$ with the same labels. The positivity expression (4.23) then should be read as the statement that the matrix formed from these states is positive definite. This is compatible with the statement that $\|\cdot\|_{\infty}$ introduced in (4.8) is actually a norm. The properties (4.22) and (4.23) are non-trivial and important in many applications. A consequence of (4.8) is that the operators $N^{2}, N^{0}$ are self-adjoint with respect to the inner product (4.8).

The complex boundary splitting. Given the decomposition (4.21) one can also determine a decomposition of the complex vector space $H^{D}\left(Y_{D}, \mathbb{C}\right)$ into $L^{2}, L_{0}$-eigenspaces, where $L^{2}=2 L_{1} L_{-1}+2 L_{-1} L_{1}+\left(L_{0}\right)^{2}$ is the Casimir operator. Let us denote the eigenvectors by $|d, l\rangle\rangle$, i.e. we require

$$
\left.\left.\left.\left.L_{0}|d, l\rangle\right\rangle=l|d, l\rangle\right\rangle, \quad L^{2}|d, l\rangle\right\rangle=d|d, l\rangle\right\rangle
$$

The important observation that we want to use in the following is the fact that there exists a transformation $\rho$, defined as

$$
\rho=\exp \frac{i \pi}{4}\left(N^{+}+N^{-}\right)=\exp \frac{i \pi}{4}\left(L_{1}+L_{-1}\right),
$$

which relates the real and complex versions of $\mathfrak{s l}(2)$ as

$$
L_{0}=\rho N^{0} \rho^{-1}, \quad L_{ \pm 1}=\rho N^{ \pm} \rho^{-1} .
$$

This can be checked by using (4.9), (4.12), and the fact that the adjoint representations satisfy $\operatorname{Ad}_{e^{X}}=e^{\operatorname{ad} x}$. Therefore, we can relate the eigenstates of $N^{2}, N^{0}$ introduced in (4.19) to the eigenstates $|d, l\rangle\rangle$ via

$$
|d, l\rangle\rangle=\rho|d, l\rangle
$$

where we recall that there can be several basis elements with the same $(d, l)$, but we have suppressed the further index labelling them. Hence we find that the complex cohomology admits a decomposition

$$
\mathcal{H}=\bigoplus_{d} \bigoplus_{l \in\{-d, \ldots, d\}} \mathcal{V}_{l}^{d}
$$

where $\mathcal{V}_{l}^{d}$ is the complex vector space spanned by the $\left.|d, l\rangle\right\rangle$.

Weights and charges of states. Having decomposed the states according as eigenstates of $L_{0}, L^{2}$, we also can add a label indicating the charge under $Q_{\infty}$. Noting that (4.15) implies $\left[Q_{\infty}, L_{0}\right]=\left[Q_{\infty}, L^{2}\right]=0$ it is possible to find simultaneous eigenstates of $L_{0}, L^{2}$ and $Q_{\infty}$ that form an orthogonal basis. We denote these by $\left.|d, l ; q\rangle\right\rangle$ with

$$
\begin{aligned}
\left.L^{2}|d, l ; q\rangle\right\rangle & =d(d+2)|d, l ; q\rangle\rangle, & d & =0, \ldots, D, \\
\left.L_{0}|d, l ; q\rangle\right\rangle & =l|d, l ; q\rangle\rangle, & l & =-d, \ldots, d, \\
\left.Q_{\infty}|d, l ; q\rangle\right\rangle & \left.=\frac{1}{2}(2 q-D)|d, l ; q\rangle\right\rangle, & & =0, \ldots, D,
\end{aligned}
$$

with $d, l, q$ being integer valued. In accordance with the CFT language, we call $l$ the weight of a state, while $q$ is the charge of the state. 


\subsection{Boundary operators and the phase operator}

In the following we will discuss the operators of the boundary theory acting on the finitedimensional Hilbert space $\mathcal{H}$. Analog to the state decomposition of subsection 4.2 we will then introduce a decomposition of operators. In addition, we will introduce a special operator $\hat{\delta}$, the phase operator, completing the data that has to be specified for the boundary theory.

Boundary splitting of operators. The operators $L^{2}, L_{0}$, and $Q_{\infty}$ introduced in the previous subsections can also be used to split the space of operators on $\mathcal{H}$. Concretely, any operator $\mathcal{O}$ acting on $\mathcal{H}$ admits an expansion ${ }^{17}$

$$
\mathcal{O}=\sum_{d, l, q \in \mathbb{Z}} \mathcal{O}_{q}^{(d, l)}
$$

with

$$
\begin{aligned}
& (\operatorname{ad} L)^{2} \mathcal{O}_{q}^{(d, l)}=d(d+2) \mathcal{O}_{q}^{(d, l)}, \quad d=0, \ldots, D \\
& {\left[L_{0}, \mathcal{O}_{q}^{(d, l)}\right]=l \mathcal{O}_{q}^{(d, l)}, \quad l=-d, \ldots, d} \\
& {\left[Q_{\infty}, \mathcal{O}_{q}^{(d, l)}\right]=q \mathcal{O}_{q}^{(d, l)}, \quad q=-D, \ldots, D \text {. }}
\end{aligned}
$$

where we have used the shorthand notation $(\operatorname{ad} L)^{2}$ to denote

$$
(\operatorname{ad} L)^{2} \mathcal{O}:=2\left[L_{1},\left[L_{-1}, \mathcal{O}\right]\right]+2\left[L_{-1},\left[L_{1}, \mathcal{O}\right]\right]+\left[L_{0},\left[L_{0}, \mathcal{O}\right]\right]
$$

We will call $l$ the weight of the operator $\mathcal{O}_{q}^{(d, l)}$ that descents from a highest weight $d$, while $q$ denotes its charge. Accordingly, from (4.15), (4.13) the operators $\left(L_{-1}, L_{0}, L_{1}\right)$ have charges $(-1,0,1)$ and weights $(-2,0,2)$ and highest weights $(2,0,2)$, respectively. Note that the adjoint operator $\mathcal{O}^{\dagger}=-C_{\infty}^{-1} \overline{\mathcal{O}} C_{\infty}$ with respect to the inner product (4.8) admits also a decomposition into $L^{2}, L_{0}$, and $Q_{\infty}$ components. Due to the fact that $C_{\infty}=i^{2 Q_{\infty}}$ we find that

$$
\left(\mathcal{O}^{\dagger}\right)_{q}^{(d, l)}=\left(\mathcal{O}_{-q}^{(d,-l)}\right)^{\dagger}
$$

It will be convenient to sometimes not perform the $L^{2}$-decomposition. We then suppress the index $d$ in (4.30) and write

$$
\mathcal{O}_{q}^{(l)} \equiv \sum_{d \in \mathbb{Z}} \mathcal{O}_{q}^{(d, l)}
$$

The space of operators with charge less than $q$ and weight less than $p$ will be denoted by ${ }^{18}$

$$
\Lambda_{q}^{(p)}=\operatorname{span}\left\{\mathcal{O}_{n}^{(s)}, n \leq q, s \leq p, r=0, \ldots, D\right\} .
$$

Note that these spaces are 'filtered' with $\Lambda_{q}^{(p)} \subset \Lambda_{\tilde{q}}^{(\tilde{p})}$ for $q \leq \tilde{q}$ and $p \leq \tilde{p}$ and that $\left[L_{-1}, \Lambda_{q}^{(p)}\right] \subset \Lambda_{q-1}^{(p-2)}$ as can be checked using (4.13) and (4.15).

\footnotetext{
${ }^{17}$ Note that this splitting corresponds to the so-called Deligne splitting of $\mathfrak{g} \mathbb{C}$ and one uses the notation $\mathcal{O}_{r, s}$, more concretely one has $\sum_{d} \mathcal{O}_{p}^{(d, p+q)}=\mathcal{O}_{p, q}$.

${ }^{18}$ Note that in [27] these spaces were denoted by $L^{p, q} \equiv \Lambda_{p}^{(p+q)}$.
} 
The phase operator. The remaining information about the boundary theory is encoded in a phase operator $\hat{\delta}$. This operator satisfies the commutation relation

$$
\left[\hat{\delta}, L_{-1}\right]=0 .
$$

It is crucial, however, that in general, $\left[\hat{\delta}, L_{1}\right] \neq 0,\left[\hat{\delta}, L_{0}\right] \neq 0$ and $\left[\hat{\delta}, Q_{\infty}\right] \neq 0$. To specify the failure of commuting with $L_{0}, Q_{\infty}$ we perform an expansion as in (4.30) with (4.34) by writing

$$
\hat{\delta}=\sum_{l, q \in \mathbb{Z}} \hat{\delta}_{q}^{(l)}
$$

$\hat{\delta}$ does not commute with $L_{0}, Q_{\infty}$ if it has components other then $\hat{\delta}_{0}^{(0)}$. To constrain $\hat{\delta}$ further we impose conditions on its components. Firstly, we require that it stems from real operator $\delta \in \mathfrak{g}_{\mathbb{R}}$ by the transformation

$$
\hat{\delta}=\rho \delta \rho^{-1}, \quad \overline{\hat{\delta}}=\rho^{-2} \hat{\delta} \rho^{2} .
$$

with $\rho$ given in (4.25). Since $\rho$ is complex, $\hat{\delta}$ is not real. Furthermore, one has to ensure that $\hat{\delta}$ is only build out of $\hat{\delta}_{q}^{(p)}$ with charge less than -1 and weight less than -2 . This amounts to the statement that $\hat{\delta}=\sum_{q \leq-1, p \leq-2} \delta_{q}^{(p)}$ or, by using (4.35) that

$$
\hat{\delta} \in \Lambda_{-1}^{(-2)} .
$$

Note that we will see later in section 5 that $\hat{\delta}$ is related to the $\delta$ introduced in (2.20) via $\hat{\delta}=\rho^{-1} \delta \rho$, when matching bulk and boundary data. In fact, the properties (4.36), (4.39) of $\hat{\delta}$ are motivated from the properties of charge operators associated to nilpotent orbits as described in appendix A.

Let us indicate the importance of the phase operator $\hat{\delta}$. It encodes a deformation of the inner product which is compatible with all the structures and is required to match a general bulk solution. In fact, one could also define another norm on the boundary that depends on $\delta$ by replacing $C_{\infty}$ in (4.8). In this case, the operation of taking the adjoint $\mathcal{O}^{\dagger}$ is no longer compatible with the eigendecomposition as in (4.33). Interestingly, this is reminiscent of the discussion of phases in the principle series representations of $S l(2, \mathbb{R})$ given in [65].

\subsection{Classification of boundary theories}

It is interesting to point out that the data that we have just specified can be used to classify allowed boundary theories. Let us consider a geometric setting with $\mathcal{H}=H^{D}\left(Y_{D}, \mathbb{C}\right)$. A first non-trivial fact that one has to use in such a classification is the identity

$$
\operatorname{dim} H^{p, q}=\operatorname{dim} H_{\infty}^{p, q} .
$$

This implies, if we restrict ourselves to Calabi-Yau manifolds, that the space $H_{\infty}^{D, 0}$ is one-dimensional and the dimension of $H_{\infty}^{D-1,1}$ counts the total number of deformations spanning the moduli space $\mathcal{M}$. The basic idea is to combine the information captured by the split (4.28) into $\mathfrak{s l}(2, \mathbb{C})$-eigenspaces with the $(p, q)$-decomposition (4.4) on the boundary. This can be done by using that $N^{-} F_{\infty}^{i} \subset F_{\infty}^{i-1}$ for $F_{\infty}^{i}$ defined analog to (2.8). 
Let us exemplify this first for Calabi-Yau threefolds $Y_{3}$ [47], which has been used in physical applications in $[3,6]$. In the threefold case the highest power of $N^{-}$acting on $H_{\infty}^{3,0}$ can be three, such that we can introduce four principle cases

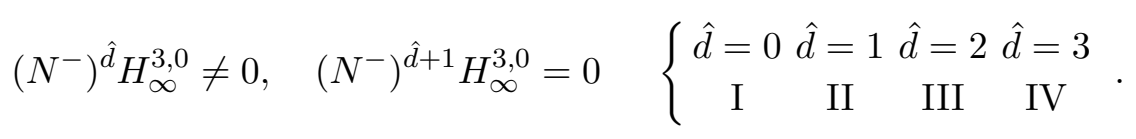

This information can be refined further by counting the number $n_{2,1}$ of elements in $H_{\infty}^{2,1}$ that are not annihilated by $N^{-}$. One records this information by a subindex leading to in total $4 h^{2,1}$ types [47]

$$
\mathrm{I}_{n_{2,1}}, \quad \mathrm{II}_{n_{2,1}-2}, \quad \mathrm{III}_{n_{2,1}-4}, \quad \mathrm{IV}_{n_{2,1}-2} .
$$

Using the decomposition (4.28) one can then infer that in the various cases the possible minimal and maximal value of $n_{2,1}$ is restricted (see $[3,6,47]$ for details). Remarkably, this classification can be mapped, using mirror symmetry, to a classification of limits in the Kähler moduli space [6, 7]. This lead recently [66] to the suggestion to classify Calabi-Yau threefolds into graphs formed by the types (4.42).

For Calabi-Yau fourfolds the classification of boundary theories proceeds in an analogous way. In this case, however, we are dealing with five principle cases, labelled by I, II, III, IV, $\mathrm{V}$ in [19], since the highest possible power $\hat{d}$ of $N^{-}$with a non-trivial action on $H_{\infty}^{4,0}$ is four. Furthermore, there are now two sub-indices to each principle case that indicate how many of the $(3,1)$ - and $(2,2)$-forms degenerate near the boundary. This data was used in [19] to study asymptotic flux compactifications. Some important subtleties in the classification of such theories for fourfolds have been pointed out in [47].

It is crucial to stress that the classification does not capture the information in $\hat{\delta}$. While it would be very interesting to include this information, this has not been done so far. In fact, it is tempting to conjecture that the boundary theory is, in fact, a theory that dynamically determines the values of $\hat{\delta}$ and enforces the properties described in section 4.3.

\section{Bulk-boundary correspondence}

In this section we have a detailed look at the matching of the boundary data introduced in section 4 with solutions to the bulk theory discussed in section 3 . The aim is to determine general solutions to the bulk equations and describe how the matching with the boundary data gives a restriction to physically viable nilpotent orbits $F_{\mathrm{pol}}^{p}$. Mathematically this matching is known as the correspondence between nilpotent orbits and limiting mixed Hodge structures $[26,27]$. The following discussion essentially summarizes some of the main steps in the proof of the $\mathrm{Sl}(2)$-obrit theorem and thus follow to large extend the seminal papers of Schmid [26] and Cattani, Kaplan, Schmid [27]. However, we will adapt a more physical language and combine some of the steps in a somewhat different manner. In particular, we aim to make the eigenspace decompositions of operators (4.31) manifest in the complete analysis. From a physics perspective the following approach of determining asymptotic solutions is a crucial part of the AdS/CFT duality and relevant, for example, for holographic renormalization [67] and bulk reconstruction [68, 69]. 
Let us recall here, for the convenience of the reader, that the bulk theory of section 3 is defined by a Hilbert space $\mathcal{H}$ with a reference inner product $\langle v \mid w\rangle_{\text {ref }}=\left\langle v, i^{2 Q} w\right\rangle$, which is determined in terms of a reference charge operator $Q$. The bulk matter fields are given by a matrix valued function $\hat{h} \in \mathfrak{g}_{\mathbb{R}}$ varying over the field space. In a one-parameter asymptotic limit $t=x+i y$, with $y \rightarrow \infty$ being the boundary, one generally has a nontrivial $y$-dependence, while the $x$-dependence is fixed by symmetry requirement (3.7) to be $\hat{h}(t, \bar{t})=e^{x N^{-}} h(y)$. In fact, a crucial information in the boundary data is the nilpotent matrix $N^{-}$, which encodes the transformation (3.7) of $\hat{h}$ under the shift $x \rightarrow x+1$. The $y$ dependence in a bulk solution $\hat{h}(t, \bar{t})$ are constrained by the equations of motion of the action (3.10). We have shown that bulk solutions $h(y)$ can be used to define three operators $\mathcal{N}^{0}(y), \mathcal{N}^{ \pm}(y) \in \mathfrak{g}_{\mathbb{R}}$ as in (3.12). These satisfy the bulk differential equations (3.3), i.e. we have

$$
(\mathrm{C} 1): \quad \partial_{y} \mathcal{N}^{ \pm}= \pm \frac{1}{2}\left[\mathcal{N}^{ \pm}, \mathcal{N}^{0}\right], \quad \partial_{y} \mathcal{N}^{0}=-\left[\mathcal{N}^{+}, \mathcal{N}^{-}\right]
$$

To extract a physical $h(y)$ from these operators, we also needed to require that the reference charge operator $Q$ acts on these solutions of (5.1) as

$$
(\mathrm{C} 2): \quad\left[Q, \mathcal{N}^{0}\right]=i\left(\mathcal{N}^{+}+\mathcal{N}^{-}\right), \quad\left[Q, \mathcal{N}^{ \pm}\right]=-\frac{i}{2} \mathcal{N}^{0}
$$

as already given in (3.4). Note that these conditions imply $\left(\mathcal{N}^{+}\right)^{\dagger}=\mathcal{N}^{-}$and $\left(\mathcal{N}^{0}\right)^{\dagger}=\mathcal{N}^{0}$, with respect to the inner product $\langle v \mid w\rangle_{\text {ref }}$ on $\mathcal{H}$ that is induced by $Q$.

In this section we will determine the general form of a solution to the bulk theory near $y=\infty$ and show how it is uniquely fixed by the boundary data. In particular, we will see that a general bulk solution takes the form

$$
\begin{gathered}
h(y)=g(\infty)\left(1+\frac{g_{1}}{y}+\frac{g_{2}}{y^{2}}+\ldots\right) y^{-\frac{1}{2} \tilde{N}^{0}}, \\
h(y)^{-1}=y^{\frac{1}{2} \tilde{N}^{0}}\left(1+\frac{f_{1}}{y}+\frac{f_{2}}{y^{2}}+\ldots\right) g(\infty)^{-1},
\end{gathered}
$$

where $\tilde{N}^{0} \in \mathfrak{g}_{\mathbb{R}}, g(\infty) \in G_{\mathbb{R}}$, and $g_{k}, f_{k}$ are operators on $\mathcal{H}$. The matrices $g_{k}, f_{k}, g(\infty)$ and $\tilde{N}^{0}$ are functions of the boundary data and can be determined, at least in principle, explicitly. The key requirement to fix the bulk data $g_{k}, g(\infty)$ and boundary data is given by

$$
e^{i \delta}=g(\infty)\left(1+\sum_{k>0} \frac{1}{k !}(-i)^{k}\left(\operatorname{ad} N^{-}\right)^{k} g_{k}\right)
$$

where we recall that $\delta$ and $N^{-}$are part of the boundary information. The matrix $\tilde{N}^{0}$ is fixed by the boundary data as

$$
\tilde{N}^{0}=g(\infty)^{-1} N^{0} g(\infty) .
$$

It is a non-trivial fact that (5.4) together with the field equations (5.1), (5.2) for $h(y)$ fixes the solution uniquely. This section is intended to explain this in detail. 
Before turning to this detailed discussion, let us note that even without giving an explicit expression for the $g_{k}, f_{k}$ and $g(\infty)$ in terms of the boundary data, we can show that these coefficients always admit several special properties that allow us to control the general behaviour of a bulk solution matching to the boundary data. In particular, one finds that

$$
\operatorname{ad}_{N^{-}}^{k+1} g_{k}=\operatorname{ad}_{N^{-}}^{k+1} f_{k}=0, \quad \hat{g}_{k}, \hat{f}_{k} \in \bigoplus_{n \leq k-1, q} \Lambda_{q}^{(n)},
$$

where we have set $\hat{g}_{k}=\rho g(\infty) g_{k} g(\infty)^{-1} \rho^{-1}$, with $\rho$ introduced in (4.25), and recall the definition (4.35) of $\Lambda_{q}^{(p)}$. We will also argue that when picking $g(\infty)$ to match the boundary data via (5.4) it can be written as

$$
g(\infty)=e^{\zeta}, \quad\left[N^{-}, \zeta\right]=0, \quad \hat{\zeta} \in \Lambda_{-1}^{(-2)},
$$

where $\hat{\zeta}=\rho \zeta \rho^{-1}$. The properties of $\zeta$ resemble those of the phase operator $\delta$ given in (4.36) and (4.39). We will see that $\hat{\zeta}$ can indeed be expressed as a universal non-commutative polynomial in the components $\hat{\delta}_{q}^{(p)}$. Taken together (5.3), (5.6), and (5.7) comprise the statements of the celebrated $\mathrm{Sl}(2)$ orbit theorem [26, 27].

\subsection{Bulk theory solutions as series expansions}

The Nahm equations (5.1) have been studied intensively in the literature. When considering $\mathcal{N}^{0}, \mathcal{N}^{ \pm}$to vary holomorphically in a complex parameter with a simple pole, a solution to (5.1) has residues that form a representation of $\mathfrak{s u}(2)$. Therefore, solutions to (5.1) naturally lead to triples, such as $N^{0}, N^{ \pm}$introduced in section 4 . In the situation at hand, we are interested in solutions in a real parameter $y$ near $y=\infty$. The general ansatz for a solution then takes the form

$$
\mathcal{N}^{0}=\frac{\tilde{N}^{0}}{y}+\mathcal{O}\left(y^{-\frac{3}{2}}\right), \quad \mathcal{N}^{ \pm}=\frac{\tilde{N}^{ \pm}}{y}+\mathcal{O}\left(y^{-\frac{3}{2}}\right) .
$$

Stated differently, we introduce $\tilde{N}^{0}, \tilde{N}^{ \pm}$satisfying a $\mathfrak{s l}(2, \mathbb{R})$ algebra

$$
\left[\tilde{N}^{0}, \tilde{N}^{ \pm}\right]= \pm 2 \tilde{N}^{ \pm}, \quad\left[\tilde{N}^{+}, \tilde{N}^{-}\right]=\tilde{N}^{0}
$$

to determine the coefficients of the slowest decreasing term of $\mathcal{N}^{0}, \mathcal{N}^{ \pm}$. Note that the leading $1 / y$ terms alone also solve (5.1), however, we will need to consider terms sub-leading in the limit $y \rightarrow \infty$ to construct a general bulk solution.

The leading coefficients $\tilde{N}^{0}, \tilde{N}^{ \pm}$will be matched with the boundary $\mathfrak{s l}(2, \mathbb{R})$ given by $N^{0}, N^{ \pm}$. Note that in general the symmetries do not need to directly and we parameterize this freedom with an element $\zeta \in \mathfrak{g}_{\mathbb{R}}$, i.e. we consider the identification

$$
N^{0}=e^{\zeta} \tilde{N}^{0} e^{-\zeta}, \quad N^{+}=e^{\zeta} \tilde{N}^{+} e^{-\zeta}, \quad N^{-}=e^{\zeta} \tilde{N}^{-} e^{-\zeta} .
$$

Clearly, such rotations preserve the algebra (5.9). Picking different $\zeta$ corresponds to picking

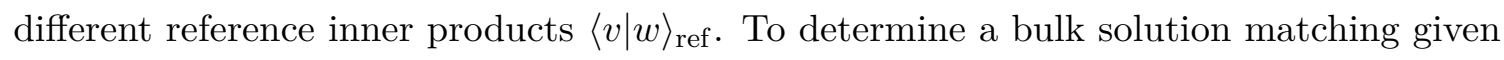
boundary data, we choose to use the reference inner product

$$
Q \equiv e^{-\zeta} Q_{\infty} e^{\zeta}, \quad\langle v \mid w\rangle_{\mathrm{ref}} \equiv\left\langle e^{-\zeta} v \mid e^{-\zeta} w\right\rangle_{\infty}
$$


with $Q_{\infty}$ and $\langle v \mid w\rangle_{\infty}$ defined on the boundary in (4.5) and (4.8), respectively. Equivalently, recalling the definition of $Q$ and $Q_{\infty}$, we can write

$$
F_{\text {ref }}^{p}=e^{-\zeta} F_{\infty}^{p}
$$

In the following we will describe how the full solution $\mathcal{N}^{0}, \mathcal{N}^{ \pm}$is fixed by the data of the boundary theory. In particular, we will see that translated back into the bulk fields $h(y)$, the solutions have the form (5.3). The element $\zeta \in \mathfrak{g}_{\mathbb{R}}$ appearing in (5.10) and (5.11) sets the overall transformation $g(\infty)$ and we will set $g(\infty)=e^{\zeta}$ later on. Indeed this matches the fact that $h(y)$ depends on the choice of reference basis $|w\rangle_{\text {ref }}$ as seen in (2.21).

To perform the analysis for this section it will turn out to be convenient to work with complex operators, analog to the relation between $N^{0}, N^{ \pm}$and $L_{\alpha}$ given in (4.26), and also rotate by the real element $\zeta$ as in (5.10), (5.11) to get a direct match with the boundary data. We thus define

$$
\mathbf{L}^{ \pm}(y):=\rho e^{\zeta} \mathcal{N}^{ \pm}(y) e^{-\zeta} \rho^{-1}, \quad \mathbf{L}^{0}(y):=\rho e^{\zeta} \mathcal{N}^{0}(y) e^{-\zeta} \rho^{-1},
$$

where

$$
\rho=\exp \frac{\pi i}{4}\left(N^{+}+N^{-}\right)=\exp \frac{\pi i}{4}\left(L_{1}+L_{-1}\right)
$$

as already defined in (4.25). With these redefinitions the differential equations (5.1) are trivially rewritten as

$$
\left(\mathrm{C} 1^{\prime}\right): \quad \partial_{y} \mathbf{L}^{ \pm}= \pm \frac{1}{2}\left[\mathbf{L}^{ \pm}, \mathbf{L}^{0}\right], \quad \partial_{y} \mathbf{L}^{0}=-\left[\mathbf{L}^{+}, \mathbf{L}^{-}\right] .
$$

Furthermore, we insert (5.11) into the conditions (5.2) and derive that

$$
\begin{aligned}
\left(\mathrm{C} 2^{\prime}\right): \quad\left[2 Q_{\infty}-L_{0}, \mathbf{L}^{0}\right] & =2 i\left(\mathbf{L}^{+}+\mathbf{L}^{-}\right)+i\left[L_{1}, \mathbf{L}^{0}\right]-i\left[L_{-1}, \mathbf{L}^{0}\right], \\
{\left[2 Q_{\infty}-L_{0}, \mathbf{L}^{ \pm}\right] } & =-i \mathbf{L}^{0}+i\left[L_{1}, \mathbf{L}^{ \pm}\right]-i\left[L_{-1}, \mathbf{L}^{ \pm}\right],
\end{aligned}
$$

where we have explicitly evaluated $\rho Q_{\infty} \rho^{-1}=Q_{\infty}-\frac{1}{2} L_{0}-\frac{i}{2} L_{1}+\frac{i}{2} L_{-1}$ by using (4.15). Consistent with (5.8) these conditions are indeed satisfied when replacing $\mathbf{L}^{ \pm}$with $L_{ \pm 1} / y$ and $\mathbf{L}^{0}$ with $L_{0} / y$. Finely, using the $\mathbf{L}^{0}, \mathbf{L}^{ \pm 1}$, we want to make sure that they stem indeed from a real function. One trivial way to implement this is to impose

$$
\left(\mathrm{C} 3^{\prime}\right): \quad \overline{\mathbf{L}}^{ \pm}=\rho^{-2} \mathbf{L}^{ \pm} \rho^{2}, \quad \overline{\mathbf{L}}^{0}=\rho^{-2} \mathbf{L}^{0} \rho^{2} .
$$

In summary, we realize that the conditions $(\mathrm{C} 1),(\mathrm{C} 2)$ for the real $\mathcal{N}^{0}, \mathcal{N}^{ \pm}$are now rewritten as $\left(\mathrm{C}^{\prime}\right),\left(\mathrm{C} 2^{\prime}\right),\left(\mathrm{C} 3^{\prime}\right)$ for the complex $\mathbf{L}^{0}, \mathbf{L}^{ \pm}$.

We next have a closer look at the full expansions of $\mathbf{L}^{0}, \mathbf{L}^{ \pm}$. We will consider the expansions of the form

$$
\mathbf{L}^{0}(y)=\sum_{n \geq 0} \frac{L_{n}^{0}}{y^{1+n / 2}}, \quad \mathbf{L}^{ \pm}(y)=\sum_{n \geq 0} \frac{L_{n}^{ \pm}}{y^{1+n / 2}} .
$$

In accordance with (5.8), (5.10), and (4.26), we fix the slowest decreasing term by the boundary conditions

$$
L_{0}^{0} \equiv L_{0}, \quad L_{0}^{ \pm} \equiv L_{ \pm 1}
$$


The goal will be to study the properties of the coefficients $L_{n}^{0}, L_{n}^{ \pm}$. In order to do that we split them into a eigen-decomposition under the commuting operators $L^{2}, L_{0}$, and $Q_{\infty}$ as in (4.30). We thus write

$$
L_{n}^{\bullet}=\sum_{q} \sum_{r>0} \sum_{-r \leq s \leq r}\left(L_{n}^{\bullet}\right)_{q}^{(r, s)}, \quad \bullet \in\{0,+,-\}
$$

with $r, s, q$ being the highest weight, the weight, and the charge of $\left(L_{n}^{\bullet}\right)_{q}^{(s, r)}$ as defined in (4.31).

The differential equation (5.15) then lead to the iterative equations

$$
\begin{aligned}
(n-s)\left(L_{n}^{+}\right)_{q+1}^{(r, s+2)} & =-\left[L_{1},\left(L_{n}^{0}\right)_{q}^{(r, s)}\right]+\sum_{0<k<n}\left[\left(L_{k}^{0}\right),\left(L_{n-k}^{+}\right)\right]_{q+1}^{(r, s+2)} \\
(n+s)\left(L_{n}^{-}\right)_{q-1}^{(r, s-2)} & =\left[L_{-1},\left(L_{n}^{0}\right)_{q}^{(r, s)}\right]+\sum_{0<k<n}\left[\left(L_{k}^{0}\right),\left(L_{n-k}^{-}\right)\right]_{q-1}^{(r, s-2)} \\
(n+2)\left(L_{n}^{0}\right)_{q}^{(r, s)} & =2\left[L_{1},\left(L_{n}^{-}\right)_{q-1}^{(r, s-2)}\right]-2\left[L_{-1},\left(L_{n}^{+}\right)_{q+1}^{(r, s+2)}\right]+2 \sum_{0<k<n}\left[\left(L_{k}^{+}\right),\left(L_{n-k}^{-}\right)\right]_{q}^{(r, s)} .
\end{aligned}
$$

In addition we can also expand the condition (5.16) in eigen-components to yield

$$
\begin{aligned}
& i(2 q-s)\left(L_{n}^{0}\right)_{q}^{(r, s)}=-2\left(L_{n}^{+}\right)_{q}^{(r, s)}-2\left(L_{n}^{-}\right)_{q}^{(r, s)}-\left[L_{1},\left(L_{n}^{0}\right)_{q-1}^{(r, s-2)}\right]+\left[L_{-1},\left(L_{n}^{0}\right)_{q+1}^{(r, s+2)}\right] \\
& i(2 q-s)\left(L_{n}^{ \pm}\right)_{q}^{(r, s)}=\left(L_{n}^{0}\right)_{q}^{(r, s)}-\left[L_{1},\left(L_{n}^{ \pm}\right)_{q-1}^{(r, s-2)}\right]+\left[L_{-1},\left(L_{n}^{ \pm}\right)_{q+1}^{(r, s+2)}\right] .
\end{aligned}
$$

Note that the weights and charges in the various terms in (5.21) and (5.22) are in accordance with the fact that $L_{ \pm 1}$ change the weight by \pm 2 and the charge by \pm 1 . Finally, the condition (5.17) decomposes as

$$
\overline{\left(L_{n}^{\bullet}\right)_{q}^{(r, s)}}=\rho^{-2}\left(L_{n}^{\bullet}\right)_{s-q}^{(r, s)} \rho^{2}
$$

where we have used that $\bar{Q}_{\infty}=-Q_{\infty}, \bar{L}_{0}=-L_{0}$, as given in (4.6), (4.14). Furthermore, we explicitly computed $\rho^{2} Q_{\infty} \rho^{-2}=Q_{\infty}-L_{0}, \rho^{2} L_{0} \rho^{-2}=-L_{0}$ and applied that $L^{2}$ is real and commutes with $\rho^{2}$. The main challenge is to extract the constraints on the coefficients $\left(L_{n}^{\bullet}\right)_{q}^{(s, r)}$ imposed by (5.21), (5.22), and (5.23).

Combining the equations (5.21) one then shows that the $\left(L_{n}\right)^{(r, s)}$ satisfy $[26,27]$

$$
\begin{aligned}
\left(L_{n}^{\bullet}\right)_{q}^{(r, s)} & =0 \quad \text { unless } \quad|s| \leq r \leq n, q \leq n-1 \\
\left(L_{n}^{\bullet}\right)_{q}^{(r, s)} & =0 \quad \text { unless } \quad(r, s, n \text { even }) \text { or }(r, s, n \text { odd }) \\
\left(L_{n}^{0}\right)_{q}^{(n, n)} & =\left(L_{n}^{0}\right)_{q}^{(n,-n)}=\left(L_{n}^{ \pm}\right)_{q}^{(n, \mp n)}=\left(L_{n}^{ \pm}\right)_{q}^{(n, \pm(2-n))}=0, \quad \text { for } n>0
\end{aligned}
$$

where one considers either the upper sign or the lower sign in each quantity. Furthermore, it turns out that all information in the expansions (5.18) satisfying the stated constraints is in the leading coefficients $\left(L_{n}^{\bullet}\right)_{q}^{(n, s)}$. Their algebra can be extracted from (5.21) by setting $r=n$. It is rather non-trivial to show that the sums over $0<k<n$ in (5.21) vanish in this 
case [27]. ${ }^{19}$ One concludes that

1. The leading coefficients $\left(L_{n}^{\bullet}\right)_{q}^{(n, s)}$ satisfy the algebra

$$
\begin{aligned}
(n-s)\left(L_{n}^{+}\right)_{q+1}^{(n, s+2)} & =-\left[L_{1},\left(L_{n}^{0}\right)_{q}^{(n, s)}\right], \\
(n+s)\left(L_{n}^{-}\right)_{q-1}^{(n, s-2)} & =\left[L_{-1},\left(L_{n}^{0}\right)_{q}^{(n, s)}\right], \\
(n+2)\left(L_{n}^{0}\right)_{q}^{(n, s)} & =2\left[L_{1},\left(L_{n}^{-}\right)_{q-1}^{(n, s-2)}\right]-2\left[L_{-1},\left(L_{n}^{+}\right)_{q+1}^{(n, s+2)}\right] .
\end{aligned}
$$

Combining this expression with $(5.22)$ the $\left(L_{n}^{\bullet}\right)_{q}^{(n, s)}$ also obey the constraint

$$
i(2 q-s)\left(L_{n}^{0}\right)_{q}^{(n, s)}=(n-s)\left(L_{n}^{+}\right)_{q}^{(n, s)}+(n+s)\left(L_{n}^{-}\right)_{q}^{(n, s)} .
$$

2. The leading coefficients $\left(L_{n}^{\bullet}\right)_{q}^{(n, s)}$ determine the solution $\mathbf{L}^{0}(y), \mathbf{L}^{ \pm 1}(y)$ satisfying (5.15), (5.16), (5.17), uniquely.

The next step is to find a solution of (5.25) fixing the leading coefficients $\left(L_{n}^{\bullet}\right)_{q}^{(n, s)}$. Remarkably, such solutions can be found to depend on one operator $\hat{\eta}=\rho \eta \rho^{-1}$ with $\eta \in \mathfrak{g}_{\mathbb{R}}$, that satisfies

$$
\hat{\eta}=\sum_{q \geq 1, n \geq 2}\left(L_{n}^{-}\right)_{-q}^{(n,-n)} \in \Lambda_{-1}^{(-2)}: \quad\left[L_{-1}, \hat{\eta}\right]=0 .
$$

One can check by straightforward computation that the equations (5.25) are satisfied by

$$
\begin{aligned}
\left(L_{n}^{0}\right)_{s-q}^{(n, 2 s-n)} & =2 a_{q}^{n, s}\left(\operatorname{ad} L_{1}\right)^{s} \hat{\eta}_{-q}^{(-n)}, \\
\left(L_{n}^{+}\right)_{s-q+1}^{(n, 2 s-n+2)} & =-(n-s)^{-1} a_{q}^{n, s}\left(\operatorname{ad} L_{1}\right)^{s+1} \hat{\eta}_{-q}^{(-n)}, \\
\left(L_{n}^{-}\right)_{s-q-1}^{(n, 2 s-n-2)} & =(n-s+1) a_{q}^{n, s}\left(\operatorname{ad} L_{1}\right)^{s-1} \hat{\eta}_{-q}^{(-n)},
\end{aligned}
$$

where $n \geq 2, s \geq 1$, and $1 \leq q \leq n-1$. Note that the complex coefficients $a_{q}^{n, s}$ are unfixed by (5.25), since these coefficients appear in each term with the same $s, q, n$ indices. To fix $a_{q}^{n, s}$ we first use (5.26) which yields the equation

$$
i(n-2 q) a_{q}^{n, s}+(n-s)(n-s+1)^{-1} a_{q}^{n, s-1}-s(n-s) a_{q}^{n, s+1}=0 .
$$

Furthermore, we also need to impose (5.23) and normalize $\eta_{-q}^{(-n)}$ such that (5.27) matches with (5.28). The former condition equates $\overline{a_{q}^{n, s}}$ with $a_{n-q}^{n, s}$ if one uses the fact that $\hat{\eta}=\rho \eta \rho^{-1}$ and $L_{1}=\rho N^{+} \rho^{-1}$ stem from real operators. The normalization condition fixes $a_{q}^{n, 1}$. We thus also have to impose the two constraints

$$
\overline{a_{q}^{n, s}}=a_{n-q}^{n, s}, \quad a_{q}^{n, 1}=\frac{1}{n} .
$$

\footnotetext{
${ }^{19}$ This follows from Proposition 6.17 of [27], which uses yet another presentation of the information in $(5.1),(5.2)$.
} 
All together the requirements (5.29), (5.30) are solved by

$$
a_{q}^{n, s}=i^{s-1} \frac{(n-s) !}{n !} b_{q-1, n-q-1}^{s-1},
$$

with integers $b_{p, q}^{k}, k, q, p \geq 0$ defined by $(1-x)^{p}(1+x)^{q}=\sum_{k} b_{p, q}^{k} x^{k}$. We check that indeed $b_{p, q}^{k}=(-1)^{k} b_{q, p}^{k}$ as required for (5.30).

To summarize, we have found that any bulk solution satisfying (5.15), (5.16), (5.17) together with the boundary conditions (5.19), is uniquely specified by a single real operator $\eta$ or $\hat{\eta}=\rho \eta \rho^{-1}$. Conversely, it is easy to extract the operator $\eta$ for a given solution via (5.27). As a next step, we will translate the solution for $\mathbf{L}^{0}, \mathbf{L}^{ \pm 1}$ into a solution for the bulk fields $h(y)$.

\subsection{Asymptotic expansions of the matter fields}

Recall that the matter fields of the bulk theory (3.22), (3.10) are the matrix-valued functions $\hat{h}(x, y)$, which asymptotically take the form $\hat{h}(x, y)=e^{x N^{-}} h(y)$. Note that any solution for $\mathcal{N}^{0}(y), \mathcal{N}^{ \pm}(y)$ satisfying (5.1), (5.2) can be parametrized by an $h(y)$ by considering

$$
\mathcal{N}^{0}(y)=-2 h^{-1} \partial_{y} h, \quad \mathcal{N}^{-}(y)=h^{-1} \tilde{N}^{-} h,
$$

where $\tilde{N}^{-}$is a real matrix that can be fixed using the boundary conditions to be $N^{-} .{ }^{20}$ We now define a new function $g(y)$ by setting

$$
h(y) \equiv g(y) y^{-\frac{1}{2} \tilde{N}^{0}},
$$

where $\tilde{N}^{0}$ is the leading coefficient in the $y$-expansion of $\mathcal{N}^{0}$ as seen in (5.8). With this definition we find that $\mathcal{N}^{0}$ takes the form

$$
\mathcal{N}^{0}(y)=-2 y^{\frac{1}{2} \tilde{N}^{0}}\left[g^{-1} D_{y} g\right] y^{-\frac{1}{2} \tilde{N}^{0}},
$$

where $D_{y}=\partial_{y}-\frac{\tilde{N}^{0}}{2 y}$.

We are now in the position to make contact to the explicit series expansions of section 5.1. In order to do that we need to implement the relation (5.13) between $\mathcal{N}^{0}$ and $\mathbf{L}^{0}$, i.e. we rotate all quantities by $\rho$, defined in (5.14), and $e^{\zeta}$. From (5.10) and (4.26) we infer that $L^{0}=\rho e^{\zeta} \tilde{N}^{0} e^{-\zeta} \rho^{-1}$. We thus can write (5.34) as

$$
\mathbf{L}^{0}(y)=-2 y^{\frac{1}{2} L^{0}}\left[\hat{g}^{-1} \hat{D}_{y} \hat{g}\right] y^{-\frac{1}{2} L^{0}}, \quad \hat{g}(y)=\rho g(y) e^{-\zeta} \rho^{-1} .
$$

Let us stress that $\hat{g}$ is not real due to the factors of $\rho$. We next use the explicit expansion (5.18) for $\mathbf{L}^{0}(y)$ and determine the expansion for $\hat{g}$. Using (5.24) we find that

$$
\hat{g}^{-1} \partial_{y} \hat{g}=\sum_{k \geq 2} \frac{B_{k}}{y^{k}}
$$

with

$$
B_{k}=-\frac{1}{2} \sum_{s \leq k-2} \sum_{r \leq 2 k-2-s} \sum_{q \leq k-2}\left(L_{2 k-2-s}^{0}\right)_{q}^{(r, s)} .
$$

\footnotetext{
${ }^{20}$ One generally checks that (5.32) satisfies (2.33), which is obtained from (5.1) and (5.2).
} 
Note that this form of $B_{k}$ implies that

$$
B_{k} \in \bigoplus_{p, q \leq k-2} \Lambda_{q}^{(p)}
$$

with $\Lambda_{q}^{(p)}$ defined in (4.35). It is important to stress that the expansion (5.36) starts with a $y^{-2}$ term, so in effect the redefinition (5.33) ensures that the $y^{-1}$-term is not present in $\hat{g}^{-1} \partial_{y} \hat{g}$ and hence not in $g^{-1} \partial_{y} g$. We can put these statements together, combine it with an argument for convergence [26,27], and infer that $g(y)$ and $g(y)^{-1}$ admit Taylor expansions at $y=\infty$ of the form $^{21}$

$$
\begin{gathered}
g(y)=g(\infty)\left(1+\frac{g_{1}}{y}+\frac{g_{2}}{y^{2}}+\ldots\right), \\
g(y)^{-1}=\left(1+\frac{f_{1}}{y}+\frac{f_{2}}{y^{2}}+\ldots\right) g(\infty)^{-1} .
\end{gathered}
$$

Note that $g(\infty)$, which is the value of $g(y)$ in the limit $y \rightarrow \infty$, drops out from (5.36) and hence is not fixed in terms of the $\left(L_{n}^{0}\right)_{q}^{(r, s)}$. We have already indicated in (5.7) that we can choose the overall transformation $e^{\zeta}=g(\infty)$, which implies that $\hat{g}, \hat{g}^{-1}$ then has the expansion

$$
\hat{g}(y)=\left(1+\frac{\hat{g}_{1}}{y}+\frac{\hat{g}_{2}}{y^{2}}+\ldots\right), \quad \hat{g}(y)^{-1}=\left(1+\frac{\hat{f}_{1}}{y}+\frac{\hat{f}_{2}}{y^{2}}+\ldots\right),
$$

with

$$
\hat{g}_{i}=\rho g(\infty) g_{i} g(\infty)^{-1} \rho^{-1}, \quad \hat{f}_{i}=\rho g(\infty) f_{i} g(\infty)^{-1} \rho^{-1} .
$$

This choice indeed normalizes the asymptotic expansion, which now depends entirely on the $\left(L_{n}^{\bullet}\right)_{q}^{(r, s)}$, which in turn are specified by $\hat{\eta}$ as discussed after $(5.27)$.

To gain a deeper understanding of the properties of the coefficients $\hat{g}_{i}$ in (5.40) we note that one can invert (5.36) to write

$$
\hat{g}_{k}=P_{k}\left(B_{2}, \ldots, B_{k+1}\right),
$$

for a set of universal non-commutatitve polynomials $P_{k}$. These polynomials $P_{k}\left(B_{2}, \ldots, B_{k+1}\right)$ are iteratively defines by

$$
P_{0}=1, \quad P_{k}=-\frac{1}{k} \sum_{j=1}^{k} P_{k-j} B_{j+1} .
$$

One checks that using (5.42) with (5.43), the differential equation $\partial_{y} \hat{g}=\hat{g} \cdot \sum_{n \geq 2} B_{n} y^{-n}$ is satisfied. Let us now recall that the $B_{n}$ are fixed in terms of the $\left(L_{n}^{0}\right)_{q}^{(r, s)}$ via (5.37). The latter are then determined from a $\hat{\eta}$ via (5.28) for the highest term and then recursively via (5.21). Taken these facts together one finds that the $\hat{g}_{k}$ (and the $\hat{f}_{k}$ ) are universal non-commutative polynomials in $L_{+1}$ and $\hat{\eta}_{p}^{(n)}$. Any appearance of $L_{0}, L_{-1}$ in the recursive evaluate can be eliminated by using the fact that $\operatorname{ad}_{L_{0}}=\left[L_{0}, \cdot\right]$ acts with integer eigenvalues

\footnotetext{
${ }^{21}$ The existence of these Taylor expansions constitute the first part of Schmid's Sl(2)-orbit theorem.
} 
on expressions involving $\hat{\eta}_{q}^{(p)}, L_{1}$, and we have $\left[L_{-1}, \hat{\eta}\right]=0$. In fact, one shows that the $\hat{g}_{k}$, $\hat{f}_{k}$ are homogeneous of degree $k$ in $\operatorname{ad}_{L_{+1}}=\left[L_{+1}, \cdot\right]$. The latter is easy to see for the leading term (5.28), and can be extend to all terms recursively [27]. It now also follows that

$$
\operatorname{ad}_{L_{-1}}^{n+1} \hat{g}_{n}=0, \quad \operatorname{ad}_{L_{-1}}^{n+1} \hat{f}_{n}=0
$$

Transformed back to $g_{i}$ we thus gets the condition (5.6), and $\operatorname{ad}_{N^{-}}^{n+1} f_{n}=0$. Furthermore, we conclude from (5.38) and (5.42) that

$$
\hat{g}_{k}, \hat{f}_{k} \in \bigoplus_{p \leq k-1, q} \Lambda_{q}^{(p)} .
$$

The properties (5.44) and (5.45) appear to be abstract, but have significant implications in concrete applications as we discuss in section 6 .

\subsection{Uniqueness of the near boundary solution}

Having determined the expansions (5.39) we next discuss a prescription how to fix the solution uniquely. In order to do this we first argue that we can bring any bulk solution into a form that is reminiscent of the form of a nilpotent orbit. Comparing this expression with the original nilpotent orbit (2.19) allows us to show that the boundary data and (5.4) are sufficient to entirely fix the bulk solution. The equation (5.4) relates the boundary data to the coefficients in the bulk solution and fixes it uniquely.

To begin with we aim to bring the bulk solution $\hat{h}(x, y)$ in a form reminiscent of a nilpotent orbit (2.19). Hence, we rewrite the information contained in a solution $\mathcal{N}^{0}=$ $-2 \hat{h}^{-1} \partial_{y} \hat{h}, \mathcal{N}^{-}=\hat{h}^{-1} \partial_{x} \hat{h}$. We first derive that

$$
h^{-1} e^{i y \tilde{N}^{-}} \frac{d}{d y}\left(e^{-i y \tilde{N}^{-}} h\right)=-i \mathcal{N}^{-}-\frac{1}{2} \mathcal{N}^{0}=-\frac{1}{2} \mathcal{L}_{0}-i \mathcal{L}_{1}
$$

where in the second equality we have used the definition (2.34) of $\mathcal{L}_{\alpha}$. We can now employ the properties of $\mathcal{L}_{\alpha}$ when acting on the reference structure $F_{\text {ref }}^{p}$. The $F_{\text {ref }}^{p}$ are complex vector spaces spanned by states with $Q$-charges being larger or equal to $p-\frac{1}{2} D$, i.e. we have $F_{\text {ref }}^{p}=\bigoplus_{r \geq p} H_{\text {ref }}^{r, D-r}$ with (2.24). Since $\left[Q, \mathcal{L}_{\alpha}\right]=\alpha \mathcal{L}_{\alpha}$, we see that $\mathcal{L}_{1}, \mathcal{L}_{0}$ preserve or increase the charge and hence conclude that $\mathcal{L}_{0} F_{\text {ref }}^{p} \subset F_{\text {ref }}^{p}$ and $\mathcal{L}_{1} F_{\text {ref }}^{p} \subset F_{\text {ref }}^{p}$ showing that $\mathcal{L}_{0}, \mathcal{L}_{1}$ preserve the vector spaces $F_{\text {ref }}^{p}$. We now read the expression (5.46) as a relation between a group element $e^{-i y \tilde{N}^{-}} h$ and an algebra element $\frac{1}{2} \mathcal{L}_{0}-\mathcal{L}_{1}$, we conclude that $e^{-i y \tilde{N}^{-}} h(y)=\kappa f(y)$, where $\kappa, f(y) \in G_{\mathbb{C}}$ with $f(y)$ preserving $F_{\text {ref }}^{p}$ and $\kappa$ being constant. Combining these last two facts we can write

$$
h(y) F_{\text {ref }}^{p}=e^{i y \tilde{N}^{-}} \tilde{F}_{0}^{p}, \quad \tilde{F}_{0}^{p}=\kappa F_{\text {ref }}^{p} .
$$

Clearly, this expression is exactly of the form (2.22), the equation which served as a definition of $h(y)$ when starting with a nilpotent orbit. Here we do not make this a priori assumption on $\hat{h}(x, y)$ and only demand that it solves the field equations (3.3), (3.4) and has the symmetry (3.7). 
The question is now to identify the conditions on $\hat{h}(x, y)$ such that $\tilde{F}^{p} \equiv e^{i y \tilde{N}^{-}} \tilde{F}_{0}^{p}$ is indeed a nilpotent orbit. This requires to enforce that $\tilde{N}^{-}$is nilpotent with $\tilde{N}^{-} \tilde{F}_{0}^{p} \subset \tilde{F}_{0}^{p-1}$ and that the Hodge decomposition $\tilde{H}^{p, q}=\tilde{F}^{p} \cap \overline{\tilde{F}^{q}}$ satisfies $\overline{\tilde{H}^{p, q}}=\tilde{H}^{q, p}$ and induces a well-defined norm. The properties of $\tilde{H}^{p, q}$ are inherited from the properties of $F_{\text {ref }}^{p}$ and we will see below how $F_{\text {ref }}^{p}$ can be matched with the boundary data. While the properties of $\tilde{N}^{-}$, such as its nilpotency, are inherited from $N^{-}$when matched as in (5.10). We conclude that indeed we can determine a nilpotent orbit from a bulk solution $h(y)$, with associated $\tilde{N}^{-}, F_{\text {ref }}^{p}$.

To complete the discussion we notice from (5.32) that $\tilde{N}^{-}=h(y) \mathcal{N}^{-}(y) h^{-1}(y)$. Inserting the explicit expansion of $h(y)$ given by (5.33), (5.39) and the expansion of $\mathcal{N}^{-}$given in (5.8), we extract the constant term yielding

$$
\tilde{N}^{-}=g(\infty) \tilde{N}^{-} g(\infty)^{-1} .
$$

Hence we find that $\left[\tilde{N}^{-}, g(\infty)\right]=0$. Compatible with this condition, we now pick

$$
g(\infty)=e^{\zeta}, \quad\left[\zeta, N^{-}\right]=0, \quad \hat{\zeta} \in \Lambda_{-1}^{(-2)},
$$

which implies that $\tilde{N}^{-}=N^{-}$. This ensures that, when re-introducing the coordinate $x$ by completing $t=x+i y$, that the nilpotent orbit derived from $h(y), F_{\text {ref }}^{p}$ transforms with the symmetry $N^{-}$associated to the boundary as in (3.7). Indeed, we can then complete (5.47) to $\hat{h}(x, y) F_{\text {ref }}^{p}=e^{t N^{-}} \tilde{F}_{0}^{p}$.

It remains to address how the boundary data fix $\zeta$, which defines $g(\infty)$ via (5.49), and $\eta$, which defines $g_{i}$ as discussed in sections 5.1, 5.2. The central statement is that for a given $N^{-}, \delta \in \mathfrak{g}_{\mathbb{R}}$, with $\left[\delta, N^{-}\right]=0, \hat{\delta} \in \Lambda_{-1}^{(-2)}$ there is a unique choice of $\zeta, \eta$ such that (5.4) is satisfied. Let us begin by motivating (5.4) by comparing the original nilpotent orbit (2.19) to the orbit (5.47). The orbit (2.19) was used to introduce a special $F_{\text {ref }}^{p}=e^{i N^{-}} e^{-i \delta} F_{0}^{p}$ in (2.20). Requiring $F_{0}^{p}=\tilde{F}_{0}^{p}$ in (5.47) we can then find the equality

$$
e^{i \delta} e^{-i N^{-}} F_{\mathrm{ref}}^{p}=e^{-i y N^{-}} h(y) F_{\mathrm{ref}}^{p} .
$$

We next turn this into an equality of vector spaces obtained from $F_{\infty}^{p}$. A key step is to realize that $F_{\infty}^{p}$ are vector spaces that are preserved by $L_{0}, L_{1}$, following an argument analog to the one after (5.46). We can then use the identity

$$
\rho=e^{i L_{-1}} e^{\frac{i}{2} L_{1}} e^{\frac{1}{\sqrt{2}} L_{0}},
$$

which implies together with (4.26) that on $F_{\infty}^{p}$ we have

$$
F_{\infty}^{p}=e^{i N^{-}} \rho^{-1} F_{\infty}^{p} .
$$

Furthermore, using the same reasoning, namely that $L_{0}$ preserves $F_{\infty}^{p}$, we have the identity $F_{\infty}^{p}=y^{\frac{1}{2} L_{0}} F_{\infty}^{p}$. Hence, using $F_{\text {ref }}^{p}=e^{-\zeta} F_{\infty}^{p}$ given in (5.12) we can thus rewrite (5.50) as

$$
\begin{aligned}
e^{i \delta} e^{-\zeta} \rho^{-1} F_{\infty}^{p} & =e^{-i y N^{-}} h(y) e^{-\zeta} e^{i N^{-}} \rho^{-1} y^{\frac{1}{2} L_{0}} F_{\infty}^{p} \\
& =e^{-i y N^{-}} g(y) y^{-\frac{1}{2} \tilde{N}^{0}} e^{-\zeta} e^{i N^{-}} y^{-\frac{1}{2} N^{0}} \rho^{-1} F_{\infty}^{p} \\
& =e^{-i y N^{-}} g(y) e^{-\zeta} y^{-\frac{1}{2} N^{0}} e^{i N^{-}} y^{\frac{1}{2} N^{0}} \rho^{-1} F_{\infty}^{p} \\
& =e^{-i y N^{-}} g(y) e^{i y N^{-}} e^{-\zeta} \rho^{-1} F_{\infty}^{p} .
\end{aligned}
$$


In the fourth identity we have used $y^{-\frac{1}{2} N^{0}} e^{i N^{-}} y^{\frac{1}{2} N^{0}}=e^{i y N^{-}}$and that $\left[\zeta, N^{-}\right]=0$. Inserting the expansion (5.39) of $g(y)$, with $g(\infty)=e^{\zeta}$, we can now evaluate

$$
e^{-i y N^{-}} g(y) e^{i y N^{-}} e^{-\zeta} \rho^{-1} F_{\infty}^{p}=e^{\zeta} \sum_{k, l \geq 0} \frac{(-i)^{k}}{k !} y^{k-l}\left(\operatorname{ad} N^{-}\right)^{k} g_{l} e^{-\zeta} \rho^{-1} F_{\infty}^{p}
$$

where we have introduced $g_{0}=1$. Due to (5.6) each term in the sum has non-positive powers and we can thus evaluate (5.54) in the limit $y \rightarrow \infty$. This leads to the identity

$$
e^{i \delta} e^{-\zeta} \rho^{-1} F_{\infty}^{p}=e^{\zeta} \sum_{k \geq 0} \frac{(-i)^{k}}{k !}\left(\operatorname{ad} N^{-}\right)^{k} g_{k} e^{-\zeta} \rho^{-1} F_{\infty}^{p} .
$$

Comparing coefficients we realize that a sufficient condition for this vector space identity to be satisfied is

$$
e^{i \delta}=e^{\zeta} \sum_{k \geq 0} \frac{(-i)^{k}}{k !}\left(\operatorname{ad} N^{-}\right)^{k} g_{k} .
$$

This is the condition (5.4) announced before and relates the boundary data $\delta, N^{-}$with the coefficients in a general bulk solution (5.3).

Let us now show that indeed the condition (5.4) is sufficient to fix the bulk solution completely when given the set of boundary data specified in section 4 . In order to do this we first transform (5.4) to

$$
e^{i \hat{\delta}} e^{-\hat{\zeta}}=\sum_{k \geq 0} \frac{(-i)^{k}}{k !}\left(\operatorname{ad} L_{-1}\right)^{k} \hat{g}_{k}
$$

where the $\hat{\delta}=\rho \delta \rho^{-1}, \hat{\zeta}=\rho \zeta \rho^{-1}$, and $\hat{g}_{i}=\rho e^{\zeta} g_{i} e^{-\zeta} \rho^{-1}$ as above. Recall from (5.42) that $\hat{g}_{k}=P_{k}\left(B_{2}, \ldots, B_{k+1}\right)$ can be expressed as a function of the coefficients $B_{l}$ appearing in (5.36) with $P_{k}$ being specific non-commutative polynomials introduced in (5.43). Using the Leibniz rule we can rewrite this expression as

$$
e^{i \hat{\delta}} e^{-\hat{\zeta}}=1+\sum_{k \geq 1} P_{k}\left(C_{2}, \ldots, C_{k+1}\right)
$$

where $C_{k+1}:=\frac{(-i)^{k}}{k !}\left(\operatorname{ad} L_{-1}\right)^{k} B_{k+1}$. We now aim to find an explicit expression for $C_{k+1}$ in terms of $\hat{\eta}$. Using the definition (5.37) of $B_{k}$ we first show ${ }^{22}$

$$
\left(\operatorname{ad} L_{-1}\right)^{k-1} B_{k}=-\frac{1}{2} \sum_{l \leq k} \sum_{q \leq k-2}\left(\operatorname{ad} L_{-1}\right)^{k-1}\left(L_{l}^{0}\right)_{q}^{(l, 2 k-2-l)}, \quad\left(\operatorname{ad} L_{-1}\right)^{k} B_{k}=0,
$$

where we have set $l=2 k-2-s$ in (5.37) and used the fact that $\operatorname{ad} L_{-1}$ lowers the weight of an operator by 2 . Furthermore, we derive by using (5.28) with (5.31) together with the $\mathfrak{s l}(2)$-algebra that

$$
\left(\operatorname{ad} L_{-1}\right)^{k}\left(L_{l}^{0}\right)_{k-q}^{(l, 2 k-l)}=2 i^{k-1} k ! b_{q-1, l-q-1}^{k-1} \hat{\eta}_{-q}^{(-l)} .
$$

\footnotetext{
${ }^{22}$ See Lemma 6.32 of [27].
} 
We are now in the position to evaluate

$$
C_{k+1}=i \sum_{l \geq k+1} \sum_{q \geq 1} b_{q-1, l-q-1}^{k-1} \hat{\eta}_{-q}^{(-l)}
$$

The formula (5.58) with (5.61) gives us an explicit expression relating $\hat{\delta}, \hat{\zeta}$ and $\hat{\eta}$. In fact we will argue next that it allows to determine $\hat{\zeta}$ and $\hat{\eta}$ as a function of $\hat{\delta}$.

To show that (5.58) with (5.61) can be used to fix $\hat{\zeta}$ and $\hat{\eta}$ in terms of $\hat{\delta}$, we note that all three operators actually stem from real counterparts $\zeta, \eta$, and $\delta$. To use this reality condition we note that it can be written as

$$
\overline{\mathcal{O}}=\rho^{-2} \mathcal{O} \rho^{2}, \quad \overline{\mathcal{O}_{q}^{(s)}}=\rho^{-2} \mathcal{O}_{s-q}^{(s)} \rho^{2}, \quad \mathcal{O} \in\{\zeta, \eta, \delta\}
$$

as we have already noted in (5.17), (5.23) for other operators. Since the identity (5.58) with (5.61) is a polynomial in the components of $\hat{\zeta}, \hat{\eta}$, and $\hat{\delta}$ the transformation involving $\rho$ simply drops on both sides. Hence, we can also replace in (5.58), (5.61):

$$
i \rightarrow-i, \quad \hat{\zeta}_{q}^{(s)} \rightarrow \hat{\zeta}_{s-q}^{(s)}, \quad \hat{\eta}_{q}^{(s)} \rightarrow \hat{\eta}_{s-q}^{(s)}, \quad \hat{\delta}_{q}^{(s)} \rightarrow \hat{\delta}_{s-q}^{(s)},
$$

and find an equally valid equation. Combined with the original expression we can then either eliminate $\hat{\eta}$ or $\hat{\zeta}$ and determine $\hat{\zeta}, \hat{\eta}$ as a function of the components of $\hat{\delta}$. For example, the first terms are

$$
\begin{array}{lll}
\hat{\zeta}_{-1}^{(-2)}=\hat{\zeta}_{-2}^{(-4)}=0, & \hat{\zeta}_{-1}^{(-3)}=-\frac{i}{2} \hat{\delta}_{-1}^{(-3)}, & \hat{\zeta}_{-1}^{(-4)}=-\frac{3 i}{4} \hat{\delta}_{-1}^{(-4)}, \\
\hat{\zeta}_{-2}^{(-5)}=-\frac{3 i}{8} \hat{\delta}_{-2}^{(-5)}-\frac{1}{8}\left[\hat{\delta}_{-1}^{(-2)}, \hat{\delta}_{-1}^{(-3)}\right], & \hat{\zeta}_{-3}^{(-6)}=-\frac{1}{8}\left[\hat{\delta}_{-1}^{(-2)}, \hat{\delta}_{-2}^{(-4)}\right] . &
\end{array}
$$

These relations suffice to treat the Calabi-Yau threefold case, but one can expand (5.58) further to determine the relations relevant for any $Y_{D}$. Note that these relations are abstractly valid and do not make use of the Calabi-Yau condition for $Y_{D}$.

Note that it remains to show that $\hat{g}_{k}$ is also fixed by the boundary data. In fact, we know from (5.42) with (5.38), (5.28) that its leading coefficients are fixed by $\hat{\eta}$, which itself is fixed by $\hat{\delta}$. The subleading coefficient are then determined by the iterative equations (5.21) and hence also involve $\operatorname{ad} L_{1} \cdot{ }^{23}$ In fact, we stress that in obtaining $\hat{\delta}$ from $\delta$ we also need $L_{1}$. We have thus argued that we can evaluate

$$
\hat{\zeta}=\hat{\zeta}(\hat{\delta}), \quad \hat{g}_{k}=\hat{g}_{k}\left(\hat{\delta}, \operatorname{ad} L_{1}\right)
$$

Note that in order to evaluate these relations it is crucial to perform the split of the operators $\hat{\delta}, \hat{\zeta}$ into weight and charge eigencomponents, which are determined by the boundary $\mathfrak{s l}(2, \mathbb{C})$-operator $L_{0}$ and $Q_{\infty}$. Hence, it is possible to evaluate using the described steps the functional dependence

$$
\zeta=\zeta\left(\delta, L_{\alpha}, Q_{\infty}\right), \quad g_{k}=g_{k}\left(\delta, L_{\alpha}, Q_{\infty}\right) .
$$

We conclude that all information about the boundary theory is needed to fix the bulk solution (5.3) with $g(\infty)=e^{\zeta}$ and $\tilde{N}^{0}=e^{-\zeta} \rho^{-1} L_{0} \rho e^{\zeta}$. Conversely, one can use a bulk solution corresponding to a nilpotent orbit to determine the boundary data.

\footnotetext{
${ }^{23}$ The dependence on $L_{0}, L_{-1}$ can be eliminated using the evaluating $L_{0}$ on the weight eigencomponents and $\left[L_{-1}, \hat{\eta}\right]=0$.
} 


\section{The finiteness of the flux landscape and the distance conjecture}

In this final section we will discuss two interesting applications of the holographic perspective developed in this work. In preparation of the physics applications we first introduce in section 6.1a powerful consequence of the detailed understanding of the near boundary expansion of the matter fields (5.3). More precisely, we will return to the analysis of the asymptotic form of the Hodge norm (2.2) and argue that the leading growth of any fixed element $F \in \mathcal{H}$ is determined by its weight decomposition under the boundary $\mathfrak{s l}(2, \mathbb{R})[26]$. This fact will be useful when studying flux compactifications and the distance conjecture. In section 6.2 we then sketch the main aspects of the proof $[39,40]$ that there are no infinite tails of flux vacua in the Type IIB or F-theory landscape near any co-dimension one boundary. The argument will be formulated for self-dual $G_{4}$ fluxes on a Calabi-Yau fourfold. We will also comment on the situation in which the fluxes are of Hodge type $(2,2)$, and note that a general proof is know for this more restrictive case. Finally, in section 6.3 we will return to the discussion of the distance conjecture, which was the initial motivation for this work. We briefly comment on how the $\mathfrak{s l}(2)$ structure on the boundary might be viewed as generalizing the original duality motivation [2] for the conjecture.

\subsection{Leading behaviour of the Hodge norm}

We want to understand how the Hodge norm $\|F\|^{2}=\int F \wedge * F$ behaves as a function of the moduli as mentioned already in the motivation of our constructions around (2.2). In the near boundary region of the boundary $t=i \infty$, we can approximate $\|F\|^{2}$ by $\|F\|_{\text {pol }}^{2}$, which amount to dropping exponentially suppressed correction $\mathcal{O}\left(e^{2 \pi i t}\right)$. This latter norm was defined in (2.14) and arises from the nilpotent orbit approximation. We have argued in the proceeding sections that $\|F\|_{\text {pol }}^{2}$ can equally be derived by using the solutions to the bulk theory that match the boundary data specified in section 4 .

The crucial outcome of the analysis of section 5 was the construction of a bulk solution matching the boundary data. This solution (5.3) relates the decomposition of forms on the boundary $H_{\infty}^{p, q}$, to the one relevant in the near boundary region. In particular, it provides us with an explicit expression of the Hodge star near the boundary as we will see in the following. Recall that we have denoted this near boundary operator by $C_{\mathrm{pol}}$ in (2.14). Concretely we find

$$
\begin{aligned}
C_{\mathrm{pol}}(t, \bar{t}) & =\hat{h} e^{-\zeta} C_{\infty} e^{\zeta} \hat{h}^{-1}, \\
& =e^{x N^{-}} h e^{-\zeta} C_{\infty} e^{\zeta} h^{-1} e^{-x N^{-}},
\end{aligned}
$$

Recall that from (5.3) with (5.5), (5.7) the solution $h(y)$ admits the expansion

$$
h(y)=e^{\zeta}\left(1+\frac{g_{1}}{y}+\frac{g_{2}}{y^{2}}+\ldots\right) e^{-\zeta} y^{-\frac{1}{2} N^{0}} e^{\zeta} .
$$

Note that $h(y) \sim y^{-\frac{1}{2} N^{0}} e^{\zeta}$ when we consider very large $y$ since all $\frac{g_{j}}{y^{j}} \rightarrow 0$. Inserted into (6.1) we thus find that

$$
C_{\mathrm{pol}}(t, \bar{t}) \sim C_{\mathrm{s}}(t, \bar{t}) \equiv e^{x N^{-}} y^{-\frac{1}{2} N^{0}} C_{\infty} y^{\frac{1}{2} N^{0}} e^{-x N^{-}} .
$$


We denote the norm corresponding to $C_{\mathrm{s}}$ by $\|\cdot\|_{\mathrm{s}}$. Hence, we find that very close to the boundary $y \rightarrow \infty$ the Hodge norm is well approximated by $[26]^{24}$

$$
\|F\|^{2} \sim\|F\|_{\mathrm{s}}^{2} \equiv\left\|y^{\frac{1}{2} N^{0}} e^{-x N^{-}} F\right\|_{\infty}^{2}=\sum_{l} y^{l}\left\|\rho_{l}\right\|_{\infty}^{2}
$$

where in the second equality we have abbreviated $\rho(x) \equiv e^{-x N^{-}} F$ and performed an eigendecomposition with respect to $N^{0}$ via

$$
\rho(x)=\sum_{l} \rho_{l}, \quad N^{0} \rho_{l}=l \rho_{l} .
$$

Note that after decomposing $\rho(x)$ we can use the orthogonality of the $N^{0}$ eigenspaces discussed in section 4 , equation (4.22), to get the result (6.4). Considering a bounded $x$, we also infer from (6.4) that

$$
\|F\|^{2} \sim \sum_{l} y^{l}\left\|F_{l}\right\|_{\infty}^{2}, \quad N^{0} F_{l}=l F_{l}
$$

where one uses that $N^{-}$acts as a lowering operator and can thus only decrease the growth. Hence, one can not lower the leading growth by tuning the field $x$. The fact that the location of $F$ in the boundary splitting of $\mathcal{H}=H^{D}\left(Y_{D}, \mathbb{C}\right)$ determine the leading growth of the Hodge norm is a well-known result of asymptotic Hodge theory [26, 27].

\subsection{Proving the finiteness of the flux landscape}

It is an important open problem in the study of flux compactifications to show that the number of 'well-defined' flux vacua is finite [35, 36]. Even in the best studied settings, namely Type IIB compactifications with three-form flux and their F-theory and M-theory generalizations, finiteness has not been fully established, even though there is compelling evidence from the analysis of the flux density [37, 38, 52, 53, 70] and individual examples [71]. Concretely, let us consider M-theory or F-theory on a Calabi-Yau fourfold $Y_{4}$, and switch on some background flux $G_{4}$. It is well-known that these fluxes are constrained by the tadpole condition $[72,73]$. Furthermore, one finds that consistency of the vacuum requires, in the absence of any non-perturbative corrections, that $G_{4}$ satisfies a self-duality condition. Together, these two conditions read $[74,75]^{25}$

$$
G_{4} \in H^{4}\left(Y_{4}, \mathbb{Z}\right): \quad\left\langle G_{4}, G_{4}\right\rangle<K, \quad G_{4}=* G_{4},
$$

where $K$ is a positive constant and we recall the definition (2.3). The finiteness of the $G_{4}$ flux landscape thus requires, as a necessary condition, that the tadpole condition and the self-duality condition have only finitely many solutions $\left(z_{\mathrm{vac}}, G_{4}\right)$ for a fixed $Y_{4}$. Here $z_{\mathrm{vac}}^{I}$

\footnotetext{
${ }^{24}$ More exactly, we can show that $\|\cdot\|$ and $\|\cdot\|_{\mathrm{s}}$ are mutually bounded, i.e. there exist positive $a_{1}, a_{2}$ such that $a_{1}\|v\|_{\mathrm{s}} \leq\|v\| \leq a_{2}\|v\|_{\mathrm{s}}$.

${ }^{25}$ In principle, one can also allow for half-quantized fluxes [76]. This does, however, not change the discussion of finiteness. Furthermore, one generally has to include a warp-factor in the dimensional reduction yielding a corrected effective action $[77,78]$. These corrections do not change the arguments made here.
} 
are choices for the complex structure moduli such that the self-duality condition is satisfied and we count the connected components in $\mathcal{M}$ parametrized by $z_{\mathrm{vac}}^{I}$, since not necessarily all $z^{I}$ might be fixed for a given $G_{4}$. The non-trivial part in answering this question lies entirely in controlling the Hodge star $*$ in (6.7) in the boundary regions of the moduli space, where it potentially blows up or decays. In the bulk of the moduli space $\mathcal{M}$ the Hodge norm $\|\cdot\|$ introduced in (2.2) is bounded and hence there only finitely many solutions to (6.7) as is apparent from $\left\langle G_{4}, G_{4}\right\rangle=\left\|G_{4}\right\|^{2}<K$ and the discreteness of the flux. Hence, the problem of showing finiteness amounts to controlling infinite tails of flux vacua. Whether or not such tails exist in certain Type IIA flux compactifications [79-81] is an ongoing debate, see e.g. [82-84].

Finiteness of supersymmetric vacua and the Hodge conjecture. Let us highlight the non-triviality of the finiteness statement. In fact, we might ask the slightly less general question if the number of supersymmetric four-form fluxes are finite. Evaluating the F-term conditions for the complex structure moduli implies that such $G_{4}$ fluxes have to be of type $(2,2)$ in the Hodge decomposition. Furthermore, demanding that the F-terms for the Kähler moduli vanish implies the primitivity of $G_{4}$. Hence, (6.7) reduces to [85]

$$
G_{4} \in H^{4}\left(Y_{4}, \mathbb{Z}\right) \cap H_{\text {prim }}^{2,2}\left(Y_{4}, \mathbb{C}\right): \quad \int_{Y_{4}} G_{4} \wedge G_{4}<K,
$$

and we can ask for finiteness of pairs $\left(z_{\mathrm{vac}}^{I}, G_{4}\right)$ satisfying these conditions. In fact, (6.8) is equivalent to the statement that $G_{4}$ is a Hodge class with bounded product. It is a famous result of Cattani, Deligne, and Kaplan [41] that the locus in complex structure moduli space at which primitive integral forms are of type $(p, p)$ is a countable union of algebraic varieties. Furthermore, they show that, if one also imposes a bound on the wedge-product as in (6.8), the number of connected components at which the fluxes are $(p, p)$ is actually finite. Applied to our situation their statement implies that the supersymmetric locus can be given by a finite number of complex algebraic equations and hence that there are only finitely many supersymmetric flux vacua. The theorems of [41] rely crucially on a clever application of the $\mathrm{Sl}(2)$-orbit theorem of [26, 27], which is also the basis of the bulk-boundary construction presented in this work. The mathematical significance of the theorems of [41] becomes eminent, when noting that the same conclusion can be obtained by applying the Hodge conjecture, which is a famously difficult problem in algebraic geometry [86]. Indeed, the Hodge conjecture can also be applied to our compactifications, since the introduced supersymmetric $G_{4}$ fluxes describe Hodge classes and simple connected Calabi-Yau manifolds are projective. The result of [41] is widely viewed as one of the strongest evidences for the Hodge conjecture.

Finiteness of self-dual vacua in one parameter limits. The one-parameter $\mathrm{Sl}(2)$ orbit theorem can equally be applied to show the finiteness statement summarize (6.7) near any one-parameter limit $[39,40]$. In fact, since the bulk-boundary construction presented here mimics the proof of the $\mathrm{Sl}(2)$-orbit theorem, the finiteness follows from the existence of a boundary theory with the properties described in section 4. More precisely, in showing that (6.7) never leads to infinite tails of vacua, one has to control the Hodge star $*$ including 
its subleading coefficients. In the following, we will sketch the proof and highlight how the results of section 5 are central in the argument. The mathematical details can be found in a letter by Schnell [39] and an upcoming work [40]. In the following we will consider a series $\left(z_{\mathrm{vac}}^{I}(n), G_{4}(n)\right)$ of solutions to (6.8) such that one of the complex structure deformations among the $z_{\mathrm{vac}}^{I}(n)$ approaches the boundary. As before we denote this field by $t$ and index the series of vacua by $n=1, \ldots, \infty$, i.e. we write

$$
t_{n}=x_{n}+i y_{n}, \quad y_{n} \rightarrow \infty \text { for } n \rightarrow \infty .
$$

while we keep $x_{n}$ bounded. We also assume that the remaining $z_{\mathrm{vac}}^{I}(n)$ are bounded. These fields play no role in our discussion and will be suppressed in the following. To justify this we note that infinite tails of vacua can only be picked up if the Hodge star diverges in a direction [37] as indicated above. This means that for a one-parameter limit $t_{n} \rightarrow i \infty$ to a co-dimension one boundary, vacua can only accumulate in this direction.

The first step in showing this result is to consider the tadpole bound (6.7) and use the self-duality of $G_{4}$ to write it using the Hodge norm

$$
K>\left\langle G_{4}(n), G_{4}(n)\right\rangle=\left\|G_{4}(n)\right\|^{2} .
$$

If we are sufficiently close to the boundary the Hodge star becomes increasingly well approximated $C_{s}$ introduced in (6.3). We thus find that (6.10) leads to the bound $\left\|G_{4}(n)\right\|_{\mathrm{S}}^{2}<K^{\prime}$ for some $K^{\prime}$. As above in (6.4) it turns out to be convenient to introduce $\rho(n)=e^{-x_{n} N^{-}} G_{4}(n)$ and to perform the decomposition $\rho(n)=\sum_{l} \rho_{l}(n)$ as in (6.5). The bound then reads

$$
K^{\prime}>\sum_{l} y_{n}^{l}\left\|\rho_{l}(n)\right\|_{\infty}^{2},
$$

which is a sum of positive terms and implies that all summands are bounded, i.e. we have

$$
K^{\prime}>\left\|\tilde{R}_{l}(n)\right\|_{\infty}^{2}, \quad \tilde{R}_{l}(n)=y_{n}^{\frac{l}{2}} \rho_{l}(n) .
$$

Here we have introduced the shorthand notation

$$
\tilde{R}(n)=\sum_{l} R_{l}(n)=y_{n}^{\frac{1}{2} N^{0}} \rho(n),
$$

which will be useful later on. Since by assumption also the axions $x_{n}$ are bounded, we conclude that $y_{n}^{l}\left\|G_{l}(n)\right\|_{\infty}^{2}$ is bounded, where $G_{l}$ are the components of $G_{4}$ in the $N^{0}$ decomposition. We can now apply the fact that the fluxes are on a lattice and hence cannot become arbitrarily small. Since $y_{n} \rightarrow \infty$ for $n \rightarrow \infty$ this means that starting at some $n^{\prime}$ the $G_{l}(n)$ with $l>0$ have to vanish. In other words we have shown that

$$
n \geq n^{\prime}: \quad G_{4}(n)=\sum_{l \leq 0} G_{l}(n), \quad \rho(n)=\sum_{l \leq 0} \rho_{l}(n), \quad \tilde{R}(n)=\sum_{l \leq 0} \tilde{R}_{l}(n),
$$

where in the last two expressions we have used that $N^{-}$lowers the $N^{0}$-eigenvalue. In the remainder of this subsection we will show that the series $\|\rho(n)\|_{\infty}$ is bounded by using the self-duality condition in (6.7). If $\|\rho(n)\|_{\infty}$ is bounded then we conclude from the 
boundedness of $x_{n}$ that also $\left\|G_{4}(n)\right\|_{\infty}$ is bounded, by using the same reasoning leading to (6.6). Recall that the norm $\|\cdot\|_{\infty}$ does not degenerate and hence we can make general statements about the boundedness of $G_{4}(n) .{ }^{26}$ Together with the fact that $G_{4}(n)$ takes values on a lattice is then enough to ensure that $G_{4}(n)$ can only take on finitely many values. Recalling that $y_{n}$ was an arbitrary path towards the boundary we conclude that there are no infinite tails towards any codimension-one boundary as we wanted to show.

Boundedness of $G_{0}(n)$. Let us first check the most straightforward case and show that the component $G_{0}(n)$ in (6.14) is bounded. This flux has a $y_{n}$-independent leading term when evaluating $\left\|G_{0}(n)\right\|$. We have argued above that all $y_{n}^{l}\left\|G_{l}(n)\right\|_{\infty}^{2}$ are bounded and hence conclude that $\left\|G_{0}(n)\right\|_{\infty}$ is bounded. This highlights again that the crucial point is to control the degeneration of the Hodge norm $\|\cdot\|$.

Boundedness in the strict asymptotic limit. It remains to show that also the $G_{l}(n)$ with $l<0$ in (6.14) are bounded. Before doing this generally, we will first focus on the situation in which we simply replace $C_{\text {nil }}$ with $C_{\mathrm{s}}$. This approximation was called strict asymptotic limit in [19] and the following finiteness result was anticipated in [19]. The self-duality condition in the leading approximation (6.3) then reads

$$
C_{\infty} \tilde{R}(n)=\tilde{R}(n)
$$

where we have used the notation (6.13). The $N^{0}$ components of $\tilde{R}(n)$ have already be introduced in (6.12). Using $C_{\infty} N^{0}=-N^{0} C_{\infty}$, which follows from $\left(N^{0}\right)^{\dagger}=-C_{\infty}^{-1} N^{0} C_{\infty}=$ $N^{0}$ given in (4.11), we have

$$
\tilde{R}_{-l}(n)=\tilde{R}_{l}(n), \quad \tilde{R}_{l}(n) \equiv y_{n}^{\frac{1}{2} l} \rho_{l}(n)
$$

where the second equality is a consequence of $N^{0} \rho_{l}=l \rho_{l}$. This fact can now be combined with our general statement (6.14) that for $n \geq n^{\prime}$ we have $\rho_{l}(n)=0$ with $l>0$. This implies that also $\rho_{l}=0$ with $l<0$ if $n \geq n^{\prime}$. Hence, we have shown that all $\rho_{l}(n), n \geq n^{\prime}$ vanish unless $l=0$. This implies that $\rho_{l}(n)$ is bounded for all values of $l$ and hence that $G_{l}(n)$ is bounded for all values of $l$.

Boundedness for the full expansion. Let us now turn to the general situation in which the Hodge star near the boundary is given by (6.1) with (6.2), when dropping exponentially suppressed corrections. We follow the argument of [39]. In this case we have to control the corrections appearing in the full expansion of $h(y)$ and $h(y)^{-1}$ in (6.1). As in the strict asymptotic case we write the self-duality condition as

$$
C_{\infty} R(n)=R(n)
$$

\footnotetext{
${ }^{26}$ The norm $\|\cdot\|_{\infty}$ can degenerate further if we hit another boundary, i.e. consider a two-parameter limit. This more general situation will not be considered here.
} 
We now have to determine $R(n)$ from the full Weil operator $C_{\text {pol }}$. Using (6.1) with (6.2) we find

$$
\begin{aligned}
R(n) & =y_{n}^{\frac{1}{2} N^{0}}\left(1+\frac{\tilde{f}_{1}}{y_{n}}+\frac{\tilde{f}_{2}}{y_{n}^{2}}+\ldots\right) \rho(n) \\
& =y_{n}^{\frac{1}{2} N^{0}}\left(1+\frac{\tilde{f}_{1}}{y_{n}}+\frac{\tilde{f}_{2}}{y_{n}^{2}}+\ldots\right) y_{n}^{-\frac{1}{2} N^{0}} \tilde{R}(n),
\end{aligned}
$$

where we have defined $\tilde{g}_{i}=e^{\zeta} g_{i} e^{-\zeta}$ and $\tilde{f}_{i}=e^{\zeta} f_{i} e^{-\zeta}$ and used the definition (6.13) of $\tilde{R}(n)$. Note that now $\tilde{R}(n)$ does not have to satisfy (6.15), since this latter condition is replaced by (6.17).

Now recall from (5.41) that $\hat{f}_{i}=\rho \tilde{f}_{i} \rho^{-1}$ and that we have argued in section 5.2, equation (5.45), that these coefficients have the special property that $\hat{f}_{k} \in \bigoplus_{p \leq k-1, q} \Lambda_{q}^{(p)}$. This implies that when expanding $\hat{f}_{k}$ into $L_{0}$ eigenvectors with $\left[L_{0}, \hat{f}_{k}^{(l)}\right]=l \hat{f}_{k}^{(l)}$ the decomposition reads $\hat{f}_{k}=\sum_{l \leq k-1} \hat{f}_{k}^{(l)}$. Rotated back to the real basis and recalling that $L_{0}=\rho N^{0} \rho^{-1}$, we find that $\tilde{f}_{k}$ has an expansion

$$
\tilde{f}_{k}=\sum_{l \leq k-1} \tilde{f}_{k}^{(l)}, \quad\left[N^{0}, \tilde{f}_{k}^{(l)}\right]=l \tilde{f}_{k}^{(l)} .
$$

These conditions come into play when evaluating (6.19). In fact, using (6.20) together with $\operatorname{Ad}_{e^{X}}=e^{\operatorname{ad} X}$ and a simple re-summation, we find

$$
R(n)=\left(1+\sum_{k \geq 0} \sum_{j=1}^{k+1} \frac{\tilde{f}_{j}^{(2 j-k-2)}}{y_{n}^{1+\frac{1}{2} k}}\right) \tilde{R}(n) .
$$

Let us now consider a $N^{0}$-component $R_{l}(n)$ with $l>0$ and use (6.14) to impose that $\tilde{R}_{l}(n)=0, l>0$ for sufficiently large $n \geq n^{\prime}$. Hence, we have

$$
R_{l}(n)=\sum_{k \geq l} \sum_{j=1}^{k+1} \frac{\tilde{f}_{j}^{(2 j-k-2)}}{y_{n}^{1+\frac{1}{2} k}} \tilde{R}_{l-(2 j-k-2)}(n) .
$$

Note that we have used here that $\tilde{R}_{r}(n)=0, r>0$ can be used to see that it suffices to consider $l-(2 j-k-2) \leq 0$ in the sum, which then sets the lower bound on the first sum. We can thus extract an overall factor $y_{n}^{-1-\frac{1}{2} l}$ and use the boundedness $(6.12)$ of $\tilde{R}_{l}(n)$ to infer the bound

$$
\left\|R_{l}(n)\right\|_{\infty} \leq B y_{n}^{-1-\frac{1}{2} l}
$$

for a sufficiently large $B \geq 0$. In order to control the coefficients $R_{l}$ with $l<0$, we use the self-duality condition (6.17). Using the same argument as for (6.16) we know that $R_{l}=R_{-l}$, and conclude that for all $l \neq 0$ we have the bound $\left\|R_{l}(n)\right\|_{\infty} \leq B y_{n}^{-1-\frac{1}{2}|l|}$. This implies that

$$
\left\|y_{n}^{-\frac{1}{2} N^{0}} R(n)\right\|_{\infty} \leq B^{\prime}
$$

where all $N^{0}$-components with $l \neq 0$ fall of with $y^{-1}$, while $R_{0}(n)$ can contain a constant term. With this bound at hand we can use (6.18) to infer that $\rho(n)$ is also bounded. Indeed, multiplying (6.18) with $y_{n}^{-\frac{1}{2} N^{0}}$ the left-hand side is bounded, while the right-hand side contains a factor $1+\sum_{i} \tilde{f}_{i} / y_{n}^{i}$ that converges to 1 in the limit $n \rightarrow \infty$. Hence $\rho(n)$ must be bounded as was required to established the above finiteness statement. 


\subsection{Comments on the distance conjecture}

The result (6.4) for the leading asymptotic of $\|F\|$ has been used intensively in $[3,6,7]$ in the study of the distance conjecture. Phrased from the holographic perspective, we can think of $F \in \mathcal{H}$ as defining a state in the boundary theory, that arises at the limit of the considered asymptotic region of moduli space. $F$ does not evolve with time, while $\rho(x)$ has some well-defined 'time' dependence. Crucially, one finds that the underlying $\mathfrak{s l}(2)$-structure, in particular the fact $\mathcal{H}$ is spanned by complete representations of $\mathfrak{s l}(2)$, dictates which states arise at any limit in moduli space. The boundary is at infinite distance, if $\mathcal{H}$ contains a state

$$
\left.\Omega_{\infty}=|\hat{d}, \hat{d} ; D\rangle\right\rangle \quad \text { with } \quad \hat{d}>0,
$$

within the splitting (4.29). To see this we use (4.40) to infer that there is only one boundary state $\Omega_{\infty}$ corresponding to the limit of the $(D, 0)$-form $\Omega$. This state is the, up to complex rescalings, unique state with charge $D$ under the charge operator $Q_{\infty}$ as inferred from (4.5). The integer $\hat{d}$ is dependent on the limit that is considered and determined by the principle type I, II, III, IV , ... introduced in section 4.4 when discussing the classification of boundary theories. The condition $\hat{d}>0$ then ensures that the limit is at infinite distance, as discussed after (2.39).

Following the arguments of $[3,6]$ one can now use $\mathfrak{s l}(2)$-representation theory, the growth behaviour (6.4), and the existence of the state (6.25) to identify candidate D-brane charges relevant to satisfy the distance conjecture. $L_{1}, L_{-1}$ or $N^{+}, N^{-}$can be used as creation and annihilation operators and raise or lower the growth by one, as inferred from the condition (6.4) and the commutation relations (4.9) and (4.13). In fact, we realize that for any state having growth $y^{l}$, with $l>0$ there always exists a state with growth $y^{-l}$. Intuitively, we can compare this to the fact that in string compactifications on a circle there always exist momentum and winding states. ${ }^{27}$ The distance conjecture was motivated in [2] by the existence of momentum and winding states in circle compactifications. The constructions of $[3,6]$ indicate that it is the underlying $\mathfrak{s l}(2)$ structure that persists in any string compactification at infinite distance points. The holographic perspective attributes the asymptotic behaviour of the field space metric and the masses of states to the existence of a boundary theory with $\mathfrak{s l}(2)$-symmetry.

Let us stress that (6.4) does not nearly exploit the complete information about the solution (6.2) that we gathered in sections 4 and 5. Specifically, we can include the subleading corrections involving the $g_{i}$. Inferring their properties (5.6) and explicit form in terms of the boundary data was central in section 5 . It is thus possible to expand $F$ into a general basis $|d, l ; q\rangle\rangle$ introduced in (4.29) and determine the complete behaviour of $\|F\|$ in the near boundary region in terms of the boundary data. Furthermore, we can also give the complete asymptotic expansion of the near boundary $(p, q)$-forms, such as $(D, 0)$-form $\Omega$. This allows one, for example, to derive general expressions for the central charge and hence extend the analysis of [19] beyond leading order. It would be interesting to do this in the future.

\footnotetext{
${ }^{27}$ Applying the above construction to $Y_{1}=T^{2}$, this interpretation can be made concrete.
} 


\section{Conclusions and further discussions}

Motivated by the recent advances in uncovering quantum gravity constraints on effective theories, we argued for a holographic approach to study the field spaces and vacua of valid effective theories. Several of the swampland conjectures, such as the distance conjecture, are constraining the behaviour of effective theories when moving to the asymptotic regions in the scalar field space. In string theory compactifications the complex structure moduli space of Calabi-Yau manifolds provides a very general example of a field space arising in consistent effective theories. Furthermore, it is known, that string dualities can relate the asymptotic regions of the complex structure moduli space to other field spaces arising, of example, at large volumes of the compactification space or at weak string coupling. Our strategy was therefore to extract the general structures arising in the asymptotic regime of the complex structure moduli space and view them as universal building blocks that should be considered abstractly and independently of their geometric realization in string compactifications. This was further motivated by the fact that asymptotic Hodge theory provides a universal and rich structure that is independent of specific geometric realizations.

In developing the holographic perspective we have first discussed several aspects of a candidate bulk theory living on the moduli space. The matter fields on the moduli space are real, group valued fields $\hat{h}$ that act on a Hilbert space that is obtained as a complexificantion of a lattice associated to the effective theory. The latter can be the charge lattice or lattice of quantized background fluxes when considering a string compactification. We have determined the dynamics of $\hat{h}$ as being given by a set of field equations and shown that they partly arise from an action principle. We have discussed this coupling of $\hat{h}$ to gravity on the moduli space with the aim to obtain as solutions to the gravity-matter system the geometric results arising in Calabi-Yau compactifications. For a real two-dimensional moduli space, this requires to go beyond Einstein gravity and we have discussed a some of the relevant field equations for the gravity-coupled matter system. Furthermore, we have made some first steps in the construction of an action principle. Our construction ensured that a particular set of solutions to the bulk theory corresponds to the nilpotent orbits and the Weil-Petersson metric after imposing appropriate boundary conditions. Nilpotent orbits are known to arise at every boundary in the Calabi-Yau moduli space, while the the Weil-Petersson metric is known to be the relevant metric in string compactifications on these spaces.

It was a central task of this work to specify boundary conditions that lead to a the set of 'physical' bulk solutions. Again we have motivated these conditions using asymptotic Hodge theory which ensures that such solutions can arise from actual geometric compactification, for example, on Calabi-Yau manifolds. The solutions admit a constrained asymptotic behaviour of the matter fields $\hat{h}$ and the metric. In particular, the metric has an asymptotic $\mathfrak{s l}(2, \mathbb{R})$ isometry, which becomes an $\mathfrak{s l}(2, \mathbb{C})$ symmetry acting on a boundary Hilbert space. The boundary Hilbert space splits under this symmetry and admits a compatible norm induced by a charge operator $Q_{\infty}$. The operator $Q_{\infty}$ induces the analog of a standard Hodge decomposition. It is non-trivial that such a decomposition can be constructed on the boundary of moduli space, since in the geometric setting the associated geometry would 
be wildly singular. This boundary data allowed us to specify three complex commuting operators: $L^{2}, L_{0}$, from the $\mathfrak{s l}(2, \mathbb{C})$, and $Q_{\infty}$. We used these to introduce quantum numbers for all states and operators. In addition we have specified an operator $\hat{\delta}$, which we termed phase operator, that encodes how the asymptotic isometry group of the metric embeds into the $\mathfrak{s l}(2, \mathbb{C})$ on the boundary. This operator is the essential part of the data which is required to perform a matching of a general bulk solution to the boundary as we discussed in section 5. It turns out that there is a single matrix condition (5.4) that fixes the bulk solution uniquely. All coefficients in the near boundary expansion of $\hat{h}$ are then fixed by universal non-commutative polynomials in $\hat{\delta}$ and the $\mathfrak{s l}(2, \mathbb{C})$ generators. The properties of these coefficients are constrained and we have shown that they are key in several applications.

It should be stressed that the precise information about the near boundary expansion makes the $S l(2)$ orbit theorem of $[26,27]$ so powerful. One of the aims of our presentation was to present the crucial parts of it proof as being part of a holographic correspondence. Furthermore, we have suggested to study string compactifications more abstractly, by extracting formal algebraic structures common to all geometric settings. This adds a new powerful way to infer general properties of the arising effective theories without the need to consider specific examples. We stress, however, that there are numerous open questions in developing the holographic correspondence further. Firstly, it would be desirable to find a complete action principle for the bulk theory. Secondly, we expect that the discussion of section 4 is only part of a more involved story about the construction of a boundary theory. Most striking would be to find a boundary theory dynamically encoding attainable values for the phase operator. Thirdly, even on the level studied here one might wonder if the holographic perspective can be generalized to higher-dimensional moduli spaces with intersecting boundary components. In mathematics this is part of the multi-variable $S l(2)$-orbit theorem which comes with several additional complications. Eventually one might hope to formulate a theory globally on the boundary. Within such a theory many aspects of the bulk physics should have boundary counterparts and one might hope for developing a dictionary for a complete bulk reconstruction in simple examples.

In the final part of this work we have discussed two finiteness results that use the existence of an $S l(2)$-structure on the boundary and the corresponding asymptotic form of the bulk solutions. Firstly, we have pointed out that a famous theorem of Cattani, Deligne, and Kaplan [41] implies the finiteness of supersymmetric flux vacua in the intensively studied F-theory compactifications with $G_{4}$ fluxes and their Type IIB analogues. The crucial task achieved in [41] is to show that the tadpole constraint ensures that there are no infinite tails of vacua near any boundary of any co-dimension. It is well-known that a similar statement can be shown by using the Hodge conjecture, which makes the study of the results of [41] into an active field of mathematical research. Secondly, we have then shown that finiteness persists, at least near co-dimension one boundaries, when considering fluxes that are self-dual and not necessarily supersymmetric. The argument uses the properties of the coefficients in the $1 / y$-expansion of the near boundary solution in order to constrain the behaviour of the Hodge star. We expect that this proof can be extended to all co-dimensions [40], hence showing finiteness of self-dual flux vacua in full generality. It should be clear, however, that several new difficulties have to be overcome, 
as it was done in the general proof of [41], which are connected to a having a much wider range of possibilities to pick a path towards the boundary.

The discussed finiteness results are of physical importance in judging the predictive power of string theory. A direct application includes the recent constructions of [87-89], in which self-dual fluxes inducing an exponentially small superpotential were introduced. Mathematically, such vacua describe certain extended loci of Hodge classes [90] and such extended loci were shown to be finite. Our arguments show that this finiteness persists even further, e.g. when the fluxes are self-dual but their induced superpotential is not exponentially small. It should be noted that the insights from the poofs of the finiteness results seem even more useful than the final statement. In particular, one learns new methods to control certain feature of scalar potentials along all possible paths in field space. Furthermore, one concretely sees why arbitrary fine-tuning is structurally prohibited. This might help, for example, to give evidence for the conjectures on moduli stabilization recently put forward in [91].

Let us close with a further speculation on how the findings of this work might yield a deeper understanding of the landscape of effective theories consistent with quantum gravity. It is natural to formulate a swampland criterium that states that every effective theory containing scalar fields admits a sector that can be described holographically with a boundary theory based on the described $S l(2)$-data. Such a proposal will then imply the following statements:

- Considering any path towards an infinite distance boundary, a continuous global symmetry becomes approximately exact that stems from a unipotent monodromy symmetry and hence can be encoded by a nilpotent $N^{-}$.

- Associated to each limit there exists a lattice $\mathcal{L}$ and a Hilbert space $\mathcal{H}=\mathbb{C} \otimes \mathcal{L}$ with an action of $N^{-}$completed into $\mathfrak{s l}(2, \mathbb{R})$. The definition of $\mathcal{H}$ and $\mathfrak{s l}(2, \mathbb{R})$ are such that (1) the positivity constraints on the effective couplings can be encoded using the norm on $\mathcal{H}$, and (2) the growth of the effective couplings in the fields sent to the limit are dictated by the $\mathfrak{s l}(2, \mathbb{R})$ weights.

Note that these statements essentially manifest the observation that there are universal constraints from positivity and the existence of global symmetry. The proposal thus claims that the structures discussed in this work are universally present. It appears to be consistent with the recent conjectures put forward in [3, 12,33,34] and the observations made in [92]. In particular, refs. $[33,34]$ view infinite distance limits as RG flows of strings, which seems nicely compatible with the holographic perspective outlined here. It would be exciting if one could develop this holographic view on the string theory landscape of effective theories further and show that many its constraining properties manifest themselves on its boundaries.

\section{Acknowledgments}

It is a great pleasure to thank Tarek Anous, Brice Bastian, Chris Couzens, Umut Gürsoy, Damian van de Heisteeg, Chongchuo Li, Jeroen Monnee, Miguel Montero, Eran Palti, Erik Plauschinn, Colleen Robles, Christian Schnell, Cumrun Vafa, and Irene Valenzuela for very 
useful discussions and correspondence. I am particularly grateful to Christian Schnell for letting me report some upcoming mathematical results on finiteness. My research is partly supported by the Dutch Research Council (NWO) via a Start-Up grant and a VICI grant. Some early parts of this work were completed at the KITP, Santa Barbara, and therefore supported in part by the National Science Foundation under Grant No. NSF PHY-1748958.

\section{A Computing the phase operator for a nilpotent orbit}

In the following we will describe how to determine the phase operator $\delta$ for a given oneparameter nilpotent orbit $F_{\mathrm{pol}}^{p}=e^{t N^{-}} F_{0}^{p}$. In contrast to the rest of the paper we will introduce in the following also the monodromy weight filtration $W_{i}$ induced by $N^{-}$. In fact, each nilpotent matrix $N^{-}$acting on $H^{D}\left(Y_{D}, \mathbb{R}\right)$ defines a unique set of real vector spaces $W_{k}\left(N^{-}\right)$of weight $D$ with

$$
0 \subset W_{0} \subset W_{1} \subset \cdots \subset W_{2 D}=H^{D}\left(Y_{D}, \mathbb{R}\right),
$$

such that for all $k$ one has

$$
N^{-} W_{k} \subset W_{k-2}, \quad\left(N^{-}\right)^{k}: \quad G r_{2 D+k} \cong G r_{2 D-k},
$$

where $G r_{k}=\frac{W_{k}}{W_{k-1}}$. The symbol $\cong$ indicates that $N^{k}$ is an isomorphism.

The set of vector spaces $W_{k}^{\mathbb{C}}=W_{k} \otimes \mathbb{C}$ together with $F_{0}^{p}$ can now be used to define the so-called Deligne splitting by setting

$$
I^{p, q}=F_{0}^{p} \cap W_{p+q}^{\mathbb{C}} \cap\left(\bar{F}_{0}^{q} \cap W_{p+q}^{\mathbb{C}}+\sum_{j \geq 1} \bar{F}_{0}^{q-j} \cap W_{p+q-j-1}^{\mathbb{C}}\right) .
$$

The $I^{p, q}$ define the unique splitting satisfying

$$
F_{0}^{p}=\bigoplus_{r \geq p} \bigoplus_{s} I^{r, s}, \quad W_{l}^{\mathbb{C}}=\bigoplus_{p+q \leq l} I^{p, q}, \quad \overline{I^{p, q}}=I^{q, p} \bmod \quad \bigoplus_{r<q, s<p} I^{r, s} .
$$

The most crucial point here, is that in general one does not find that $\overline{I^{p, q}}=I^{q, p}$. We can now define vector spaces $V_{l}$ and a semisimple grading operator

$$
V_{l}^{\mathbb{C}}=\bigoplus_{p+q=l} I^{p, q}, \quad T v_{l}=l v_{l} \text { for } v_{l} \in V_{l}^{\mathbb{C}} .
$$

The operator $T$ should be compared with the operator $N^{0}$ introduced in section 4 . However, while $\bar{N}^{0}=N^{0}$, this is not necessarily the case for $T$, since in general $\bar{V}_{l}^{\mathbb{C}} \neq V_{l}^{\mathbb{C}}$. In other words, there is in general no real slice in $V_{l}^{\mathbb{C}}$ on which $T$ acts as $l$ and which yields the space $V_{l}^{\mathbb{C}}$ as complexification. This is in stark contrast to the vector spaces (4.28) introduced on the boundary. We will now describe that there how to construct the unique rotation of $T$, such that a real split exists.

To being with, let $\bar{T}$ be the complex conjugate of the grading operator $T$ defined by $\bar{T}(v):=\overline{T(\bar{v})}$, for all $v \in H^{D}\left(Y_{D}, \mathbb{C}\right)$. One can now show [27] that $\bar{T}$ and $T$ are related by

$$
\bar{T}=e^{-2 \mathbf{i} \delta} T e^{2 \mathbf{i} \delta}
$$


where the real operator $\delta$ acts on $I^{p, q}$ by decreasing $p, q$, i.e.

$$
\delta\left(I^{p, q}\right) \subset \bigoplus_{\substack{r<p \\ s<q}} I^{r, s},
$$

holding for all $p, q$. Requiring that $\delta \in \mathfrak{g}_{\mathbb{R}}$ and that $\left[N^{-}, \delta\right]=0$, one shows that there is a unique operator $\delta$ satisfying (A.6) and (A.7) (see Proposition 2.20 of [27] for details). For any given nilpotent orbit we can thus compute the unique charge operator $\delta$. Simple example for such a computation can be found e.g. in [6].

Open Access. This article is distributed under the terms of the Creative Commons Attribution License (CC-BY 4.0), which permits any use, distribution and reproduction in any medium, provided the original author(s) and source are credited.

\section{References}

[1] E. Palti, The Swampland: Introduction and Review, Fortsch. Phys. 67 (2019) 1900037 [arXiv: 1903.06239] [INSPIRE].

[2] H. Ooguri and C. Vafa, On the Geometry of the String Landscape and the Swampland, Nucl. Phys. B 766 (2007) 21 [hep-th/0605264] [INSPIRE].

[3] T.W. Grimm, E. Palti and I. Valenzuela, Infinite Distances in Field Space and Massless Towers of States, JHEP 08 (2018) 143 [arXiv: 1802.08264] [INSPIRE].

[4] S.-J. Lee, W. Lerche and T. Weigand, Tensionless Strings and the Weak Gravity Conjecture, JHEP 10 (2018) 164 [arXiv:1808.05958] [INSPIRE].

[5] S.-J. Lee, W. Lerche and T. Weigand, A Stringy Test of the Scalar Weak Gravity Conjecture, Nucl. Phys. B 938 (2019) 321 [arXiv:1810.05169] [InSPIRE].

[6] T.W. Grimm, C. Li and E. Palti, Infinite Distance Networks in Field Space and Charge Orbits, JHEP 03 (2019) 016 [arXiv: 1811.02571] [INSPIRE].

[7] P. Corvilain, T.W. Grimm and I. Valenzuela, The Swampland Distance Conjecture for Kähler moduli, JHEP 08 (2019) 075 [arXiv: 1812.07548] [INSPIRE].

[8] A. Font, A. Herráez and L.E. Ibáñez, The Swampland Distance Conjecture and Towers of Tensionless Branes, JHEP 08 (2019) 044 [arXiv: 1904.05379] [INSPIRE].

[9] S.-J. Lee, W. Lerche and T. Weigand, Emergent Strings, Duality and Weak Coupling Limits for Two-Form Fields, arXiv: 1904.06344 [INSPIRE].

[10] F. Marchesano and M. Wiesner, Instantons and infinite distances, JHEP 08 (2019) 088 [arXiv: 1904.04848] [INSPIRE].

[11] T.W. Grimm and D. Van De Heisteeg, Infinite Distances and the Axion Weak Gravity Conjecture, JHEP 03 (2020) 020 [arXiv: 1905.00901] [INSPIRE].

[12] S.-J. Lee, W. Lerche and T. Weigand, Emergent Strings from Infinite Distance Limits, arXiv: 1910.01135 [INSPIRE].

[13] F. Baume, F. Marchesano and M. Wiesner, Instanton Corrections and Emergent Strings, JHEP 04 (2020) 174 [arXiv: 1912. 02218] [INSPIRE]. 
[14] M. Enríquez Rojo and E. Plauschinn, Swampland conjectures for type IIB orientifolds with closed-string U(1)s, JHEP 07 (2020) 026 [arXiv: 2002.04050] [INSPIRE].

[15] N. Gendler and I. Valenzuela, Merging the weak gravity and distance conjectures using BPS extremal black holes, JHEP 01 (2021) 176 [arXiv:2004.10768] [INSPIRE].

[16] B. Heidenreich and T. Rudelius, Infinite Distance and Zero Gauge Coupling in 5d Supergravity, arXiv: 2007.07892 [INSPIRE].

[17] T. Banks and L.J. Dixon, Constraints on String Vacua with Space-Time Supersymmetry, Nucl. Phys. B 307 (1988) 93 [INSPIRE].

[18] T. Banks and N. Seiberg, Symmetries and Strings in Field Theory and Gravity, Phys. Rev. D 83 (2011) 084019 [arXiv: 1011.5120] [INSPIRE].

[19] T.W. Grimm, C. Li and I. Valenzuela, Asymptotic Flux Compactifications and the Swampland, JHEP 06 (2020) 009 [Erratum ibid. 01 (2021) 007] [arXiv: 1910.09549] [INSPIRE].

[20] E. Cattani and A. Kaplan, Degenerating variations of Hodge structure, in Théorie de Hodge Luminy, Juin 1987, Barlet D. and Esnault H. and Elzein F. and Verdier Jean-Louis and Viehweg E. eds, Societé mathématique de France, Astérisque 179-180 (1989).

[21] E. Cattani, F.E. Zein, P.A. Griffiths and L.D. Tráng, Hodge Theory (MN-49), Princeton University Press, Princeton U.S.A (2014) [ISBN: 9780691161341].

[22] K. Hori et al., Mirror symmetry, AMS, Clay Math. Monogr. 1, Providence U.S.A. (2003) [INSPIRE].

[23] M. Kerr and R. Laza, Hodge theory of degenerations, (i): Consequences of the decomposition theorem, arXiv:1901.01896.

[24] M. Kerr and R. Laza, Hodge theory of degenerations, (ii): vanishing cohomology and geometric applications, arXiv:2006.03953.

[25] C.-L. Wang, On the incompleteness of the Weil-Petersson metric along degenerations of Calabi-Yau manifolds, Math. Res. Lett. 4 (1997) 157.

[26] W. Schmid, Variation of Hodge structure: the singularities of the period mapping, Invent. Math. 22 (1973) 211.

[27] E. Cattani, A. Kaplan and W. Schmid, Degeneration of Hodge Structures, Annals Math. 123 (1986) 457.

[28] S.K. Donaldson, Nahm's equations and the classification of monopoles, Commun. Math. Phys. 96 (1984) 387 [INSPIRE].

[29] S. Cecotti and C. Vafa, Topological antitopological fusion, Nucl. Phys. B 367 (1991) 359 [INSPIRE].

[30] S. Cecotti, Special Geometry and the Swampland, JHEP 09 (2020) 147 [arXiv:2004.06929] [INSPIRE].

[31] S. Cecotti, Moduli spaces of Calabi-Yau d-folds as gravitational-chiral instantons, JHEP 12 (2020) 008 [arXiv: 2007.09992] [INSPIRE].

[32] P. Deligne, Structures de Hodge mixtes réelles, Proc. Sympos. Pure Math. 55 (1994) 509.

[33] S. Lanza, F. Marchesano, L. Martucci and I. Valenzuela, Swampland Conjectures for Strings and Membranes, JHEP 02 (2021) 006 [arXiv: 2006. 15154] [INSPIRE].

[34] S. Lanza, F. Marchesano, L. Martucci and I. Valenzuela, to appear. 
[35] M. Graña, Flux compactifications in string theory: A Comprehensive review, Phys. Rept. 423 (2006) 91 [hep-th/0509003] [INSPIRE].

[36] M.R. Douglas and S. Kachru, Flux compactification, Rev. Mod. Phys. 79 (2007) 733 [hep-th/0610102] [INSPIRE].

[37] S. Ashok and M.R. Douglas, Counting flux vacua, JHEP 01 (2004) 060 [hep-th/0307049] [INSPIRE].

[38] F. Denef and M.R. Douglas, Distributions of flux vacua, JHEP 05 (2004) 072 [hep-th/0404116] [INSPIRE].

[39] C. Schnell, Letter to T. Grimm (2020).

[40] T.W. Grimm and C. Schnell, in preparation.

[41] E. Cattani, P. Deligne and A. Kaplan, On the locus of Hodge classes, J. Am. Math .Sci. 8 (1995) 483 [alg-geom/9402009].

[42] H. Hironaka, Resolution of Singularities of an Algebraic Variety Over a Field of Characteristic Zero: I, Annals Math. 79 (1964) 109.

[43] E. Viehweg, Quasi-projective Moduli for Polarized Manifolds,Springer Berlin (1995) [DOI].

[44] E. Palti, C. Vafa and T. Weigand, Supersymmetric Protection and the Swampland, JHEP 06 (2020) 168 [arXiv: 2003.10452] [INSPIRE].

[45] B. Bastian, T.W. Grimm and D. v. d. Heisteeg, in preparation.

[46] C. Robles, Classification of horizontal SL(2)s, Compos. Math. 152 (2016) 918 [arXiv: 1405.3163].

[47] M. Kerr, G.J. Pearlstein and C. Robles, Polarized relations on horizontal SL(2)'s, Doc. Math. 24 (2019) 1295.

[48] Z. Lu, On the geometry of classifying spaces and horizontal slices, Amer. J. Math. 121 (1999) 177 [math/0505579].

[49] Z. Lu, On the Hodge metric of the universal deformation space of Calabi-Yau threefolds, J. Geom. Anal. 11 (2001) 103.

[50] Z. Lu and X. Sun, Weil-Petersson geometry on moduli space of polarized Calabi-Yau manifolds, J. Inst. Math. Jussieu 3 (2004) 185.

[51] H. Fang and Z. Lu, Generalized Hodge metrics and BCOV torsion on Calabi-Yau moduli, J. reine und angewandte Math. 588 (2005) 49 [math/0310007].

[52] M. Douglas and Z. Lu, On the geometry of moduli space of polarized Calabi-Yau manifolds, math/0603414 [INSPIRE].

[53] Z. Lu and M.R. Douglas, Gauss-Bonnet-Chern theorem on moduli space, Math. Ann. 357 (2013) 469 [arXiv: 0902.3839] [INSPIRE].

[54] C. Peters and G. Pearlstein, Differential geometry of the mixed hodge metric, Commun. Anal. Geom. 3 (2019) 671 [arXiv:1407.4082].

[55] M. Bershadsky, S. Cecotti, H. Ooguri and C. Vafa, Kodaira-Spencer theory of gravity and exact results for quantum string amplitudes, Commun. Math. Phys. 165 (1994) 311 [hep-th/9309140] [INSPIRE]. 
[56] Z. Lu, On the Hodge Metric of the Universal Deformation Space of Calabi-Yau Threefolds, J. Geom. Anal. 11 (2005) 103 [math/0505582].

[57] T.W. Grimm, D. v. d. Heisteeg and J. Monnee, Bulk reconstruction in moduli space holography, (2021) [arXiv:2103.12746].

[58] N.J. Hitchin, On the Construction of Monopoles, Commun. Math. Phys. 89 (1983) 145 [INSPIRE].

[59] C. Teitelboim, Gravitation and Hamiltonian Structure in Two Space-Time Dimensions, Phys. Lett. B 126 (1983) 41 [inSPIRE].

[60] R. Jackiw, Lower Dimensional Gravity, Nucl. Phys. B 252 (1985) 343 [InSPIRE].

[61] D. Grumiller, W. Kummer and D.V. Vassilevich, Dilaton gravity in two-dimensions, Phys. Rept. 369 (2002) 327 [hep-th/0204253] [INSPIRE].

[62] D. Grumiller and R. Meyer, Ramifications of lineland, Turk. J. Phys. 30 (2006) 349 [hep-th/0604049] [INSPIRE].

[63] V. de Alfaro, S. Fubini and G. Furlan, Conformal Invariance in Quantum Mechanics, Nuovo Cim. A 34 (1976) 569 [inSPIRE].

[64] C. Chamon, R. Jackiw, S.-Y. Pi and L. Santos, Conformal quantum mechanics as the CFT dual to $A d S_{2}$, Phys. Lett. B 701 (2011) 503 [arXiv:1106.0726] [INSPIRE].

[65] D. Anninos, D.M. Hofman and J. Kruthoff, Charged Quantum Fields in AdS $S_{2}$, SciPost Phys. 7 (2019) 054 [arXiv: 1906.00924] [InSPIRE].

[66] T.W. Grimm, F. Ruehle and D. van de Heisteeg, Classifying Calabi-Yau Threefolds Using Infinite Distance Limits, Commun. Math. Phys. 382 (2021) 239 [arXiv:1910. 02963] [INSPIRE].

[67] K. Skenderis, Lecture notes on holographic renormalization, Class. Quant. Grav. 19 (2002) 5849 [hep-th/0209067] [inSPIRE].

[68] T. De Jonckheere, Modave lectures on bulk reconstruction in AdS/CFT, PoS Modave2017 (2018) 005 [arXiv: 1711.07787] [INSPIRE].

[69] D. Harlow, TASI Lectures on the Emergence of Bulk Physics in AdS/CFT, PoS TASI2017 (2018) 002 [arXiv: 1802.01040] [INSPIRE].

[70] T. Eguchi and Y. Tachikawa, Distribution of flux vacua around singular points in Calabi-Yau moduli space, JHEP 01 (2006) 100 [hep-th/0510061] [INSPIRE].

[71] A.P. Braun, N. Johansson, M. Larfors and N.-O. Walliser, Restrictions on infinite sequences of type IIB vacua, JHEP 10 (2011) 091 [arXiv:1108.1394] [INSPIRE].

[72] M.J. Duff, J.T. Liu and R. Minasian, Eleven-dimensional origin of string-string duality: A One loop test, Nucl. Phys. B 452 (1995) 261 [hep-th/9506126] [InSPIRE].

[73] S. Sethi, C. Vafa and E. Witten, Constraints on low dimensional string compactifications, Nucl. Phys. B 480 (1996) 213 [hep-th/9606122] [INSPIRE].

[74] K. Becker and M. Becker, M theory on eight manifolds, Nucl. Phys. B 477 (1996) 155 [hep-th/9605053] [INSPIRE].

[75] K. Dasgupta, G. Rajesh and S. Sethi, M theory, orientifolds and G - flux, JHEP 08 (1999) 023 [hep-th/9908088] [INSPIRE]. 
[76] E. Witten, On flux quantization in M-theory and the effective action, J. Geom. Phys. 22 (1997) 1 [hep-th/9609122] [INSPIRE].

[77] T.W. Grimm, T.G. Pugh and M. Weissenbacher, The effective action of warped M-theory reductions with higher derivative terms - part I, JHEP 01 (2016) 142 [arXiv:1412.5073] [INSPIRE].

[78] T.W. Grimm, T.G. Pugh and M. Weissenbacher, The effective action of warped M-theory reductions with higher-derivative terms - Part II, JHEP 12 (2015) 117 [arXiv:1507.00343] [INSPIRE].

[79] T.W. Grimm and J. Louis, The Effective action of type IIA Calabi-Yau orientifolds, Nucl. Phys. B 718 (2005) 153 [hep-th/0412277] [InSPIRE].

[80] J.-P. Derendinger, C. Kounnas, P.M. Petropoulos and F. Zwirner, Superpotentials in IIA compactifications with general fluxes, Nucl. Phys. B 715 (2005) 211 [hep-th/0411276] [INSPIRE].

[81] O. DeWolfe, A. Giryavets, S. Kachru and W. Taylor, Type IIA moduli stabilization, JHEP 07 (2005) 066 [hep-th/0505160] [INSPIRE].

[82] D. Junghans, O-Plane Backreaction and Scale Separation in Type IIA Flux Vacua, Fortsch. Phys. 68 (2020) 2000040 [arXiv: 2003.06274] [INSPIRE].

[83] G. Buratti, J. Calderon, A. Mininno and A.M. Uranga, Discrete Symmetries, Weak Coupling Conjecture and Scale Separation in AdS Vacua, JHEP 06 (2020) 083 [arXiv:2003.09740] [INSPIRE].

[84] F. Marchesano, E. Palti, J. Quirant and A. Tomasiello, On supersymmetric AdS $S_{4}$ orientifold vacua, JHEP 08 (2020) 087 [arXiv:2003.13578] [INSPIRE].

[85] S. Gukov, C. Vafa and E. Witten, CFT's from Calabi-Yau four folds, Nucl. Phys. B 584 (2000) 69 [Erratum ibid. 608 (2001) 477] [hep-th/9906070] [INSPIRE].

[86] P. Deligne, The Hodge conjecture, Clay Math. Inst., The Millennium Prize Problems (2006), pp. $45-53$.

[87] M. Demirtas, M. Kim, L. Mcallister and J. Moritz, Vacua with Small Flux Superpotential, Phys. Rev. Lett. 124 (2020) 211603 [arXiv:1912.10047] [INSPIRE].

[88] M. Demirtas, M. Kim, L. McAllister and J. Moritz, Conifold Vacua with Small Flux Superpotential, Fortsch. Phys. 68 (2020) 2000085 [arXiv: 2009.03312] [InSPIRE].

[89] R. Álvarez-García, R. Blumenhagen, M. Brinkmann and L. Schlechter, Small Flux Superpotentials for Type IIB Flux Vacua Close to a Conifold, arXiv:2009.03325 [INSPIRE].

[90] C. Schnell, The extended locus of Hodge classes, arXiv:1401.7303.

[91] I. Bena, J. Blåbäck, M. Graña and S. Lüst, The Tadpole Problem, arXiv:2010.10519 [INSPIRE].

[92] S. Andriolo, T.-C. Huang, T. Noumi, H. Ooguri and G. Shiu, Duality and axionic weak gravity, Phys. Rev. D 102 (2020) 046008 [arXiv:2004.13721] [INSPIRE]. 